\title{
ACCURACY OF FOREST ROAD AND STREAM CHANNEL CHARACTERISTICS DERIVED FROM LIDAR IN FORESTED MOUNTAIN CONDITIONS
}

\author{
A Thesis \\ Presented to the Faculty of \\ California Polytechnic State University \\ San Luis Obispo
}

\author{
In Partial Fulfillment \\ of the Requirements for the Degree \\ Master of Science in Forestry Sciences \\ by \\ Russell Alan White
}

March 2010 
(C) 2010

Russell Alan White

ALL RIGHTS RESERVED 


\section{COMMITTEE MEMBERSHIP}

TITLE:

ACCURACY OF FOREST ROAD AND STREAM CHANNEL CHARACTERISTICS DERIVED FROM LIDAR IN FORESTED MOUNTAIN CONDITIONS

AUTHOR: $\quad$ Russell Alan White

DATE SUBMITTED: $\quad$ March 2010

COMMITTEE CHAIR: $\quad$ Brian C. Dietterick, Ph.D., P.H.

Professor of Hydrology and Watershed Management

COMMITTEE MEMBER: $\quad$ Norman H. Pillsbury, Ph.D., RPF

Professor of Forestry and Hydrology

COMMITTEE MEMBER: $\quad$ Samantha J. Gill, Ph.D., RPF

Professor of Forest Measurements and Management 


\author{
ABSTRACT \\ Accuracy of Forest Road and Stream Channel Characteristics \\ Derived from LiDAR in Forested Mountain Conditions \\ Russell White
}

Forest roads and stream channels are mapped using a variety of remote sensing and ground-based techniques. In densely forested areas, conventional remote sensing methods provide limited terrain information, while ground-based surveys can be time-consuming, difficult, and expensive. Light Detection and Ranging (LiDAR) is an airborne remote sensing technology used to create high-resolution digital elevation models (DEMs) of the earth's surface. This study tests the accuracy of forest road and stream channel features mapped using LiDAR in the steep, forested terrain of California's Santa Cruz Mountains. A conventional total station survey was used to determine centerline position and elevations along a four-kilometer forest road, and along six thirty-meter stream channel study reaches. A $1.5 \mathrm{~m} \mathrm{LiDAR} \mathrm{DEM} \mathrm{was}$ suitable to accurately map the location of the forest road and channel features. Ninety five percent of the LiDAR-derived road length was located within $2.2 \mathrm{~m}$ normal to the field-surveyed centerline and LiDAR-derived road slopes were not significantly different from field-surveyed slopes. Stream channel features derived from the LIDAR DEM were located within $2.7 \mathrm{~m}$ normal to the field-surveyed thalweg, while the LiDAR-derived slopes measured within 0.49 percent of field-surveyed slopes. These findings indicate that LiDAR can provide accurate terrain measurements that are suitable for resource management and assessment. 


\section{ACKNOWLEDGEMENTS}

I thank my advisor, Brian Dietterick, for the opportunity to explore this topic, and for his guidance toward this final product. My thanks also to Tom Mastin and Rollin Strohman for lending their considerable expertise and practical advice. During this project, I have also enjoyed the opportunity to live and work as a resident graduate student at Swanton Pacific Ranch. My deep appreciation and thanks go to the faculty, staff, and students who have all worked to realize the unique mission at Swanton. Finally, I thank my family for their love, support, and encouragement in my education, and in following greater aspirations in life. 
TABLE OF CONTENTS

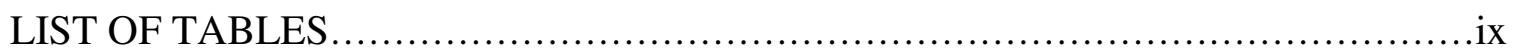

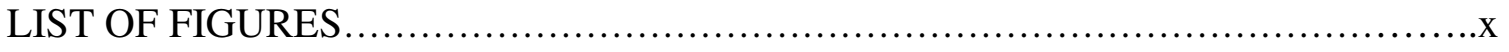

CHAPTER

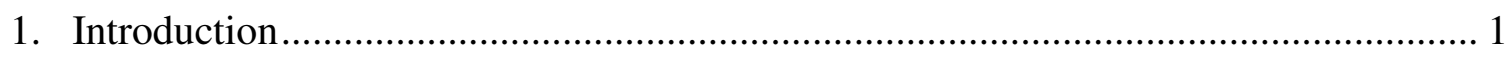

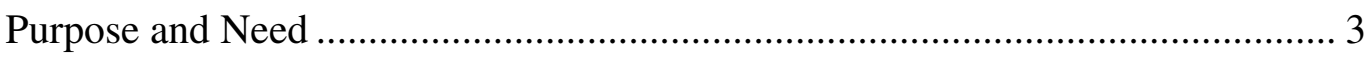

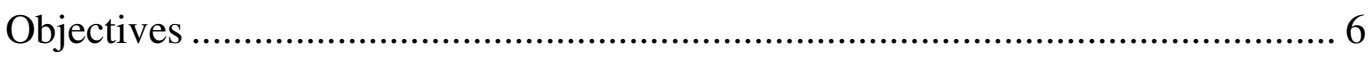

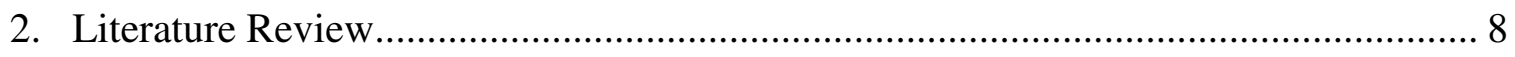

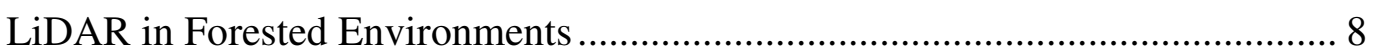

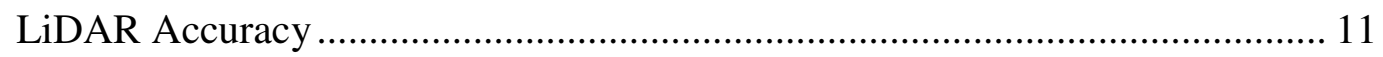

Applications for Watershed Management and Assessment................................ 16

Applications for Mapping Forest Roads ........................................................... 18

Applications in Hydrology and Geomorphology .............................................. 21

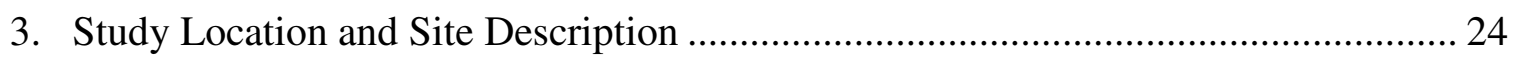

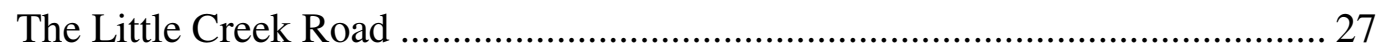

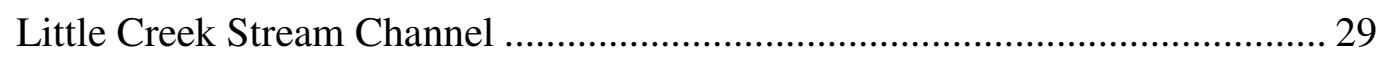

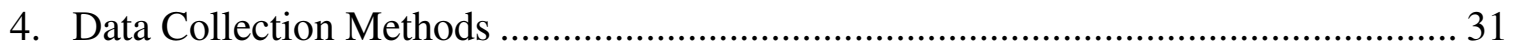

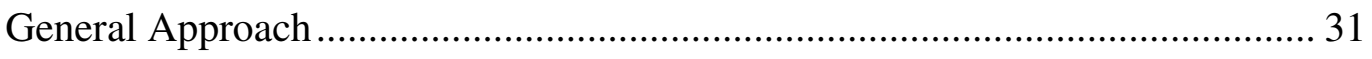

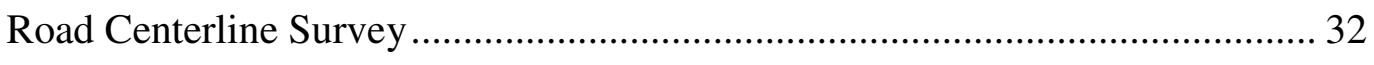

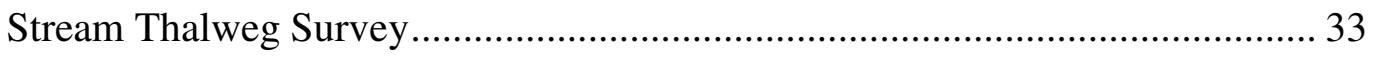

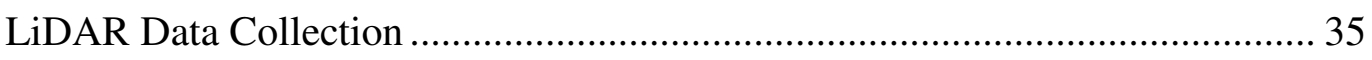

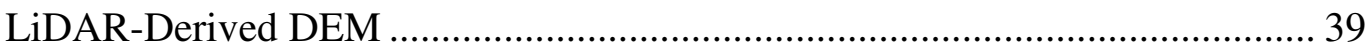


Digitized Forest Road Location ............................................................. 41

Derived Stream Channel Location.............................................................. 46

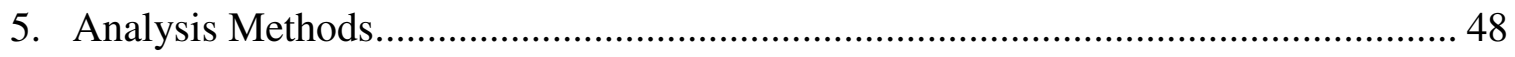

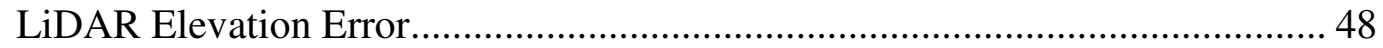

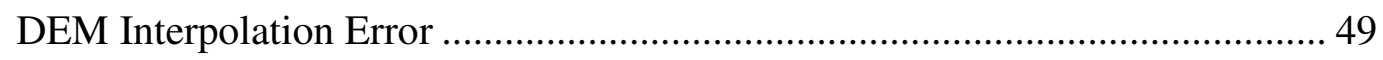

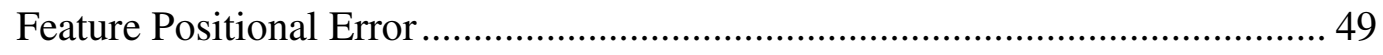

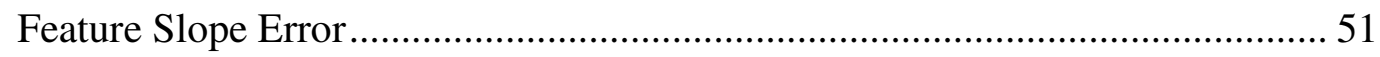

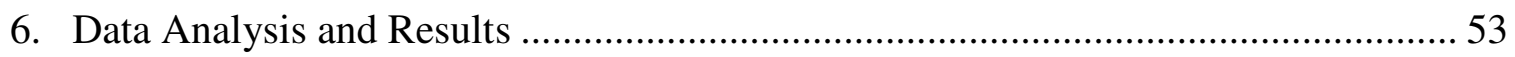

LiDAR Elevation Error.................................................................................. 53

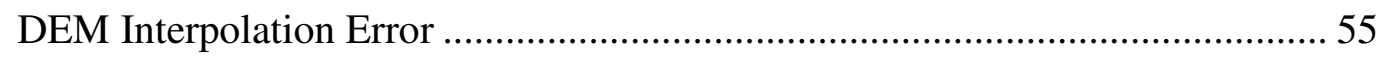

Evaluating LiDAR-Derived Features ..................................................... 58

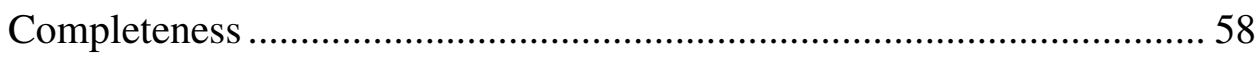

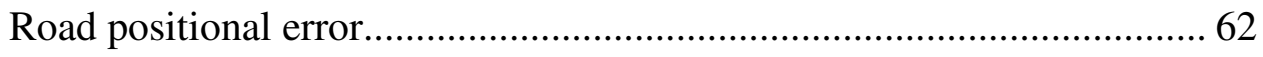

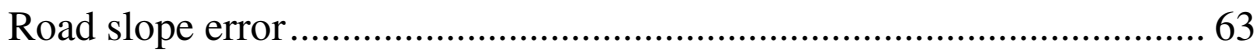

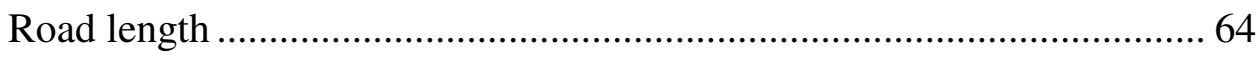

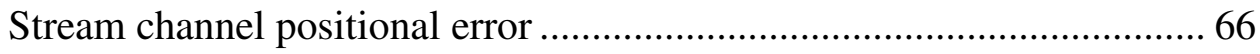

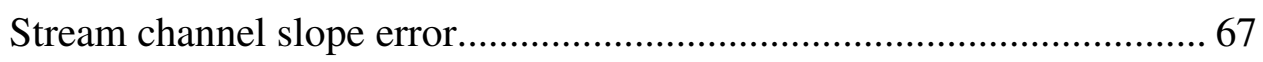

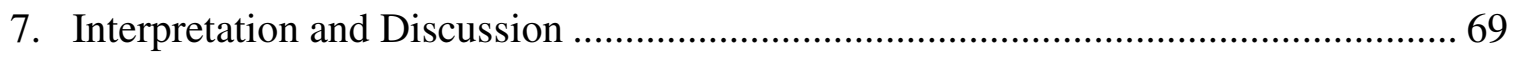

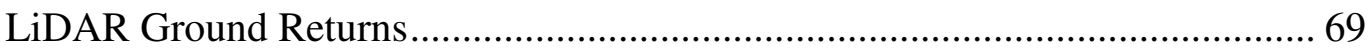

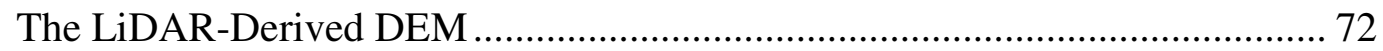

LiDAR-Derived Road Measurements ....................................................... 74

LiDAR-Derived Stream Channel Measurements ........................................... 79

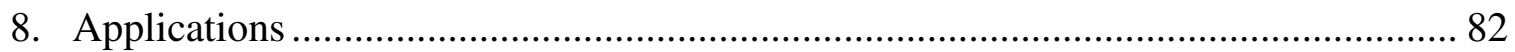




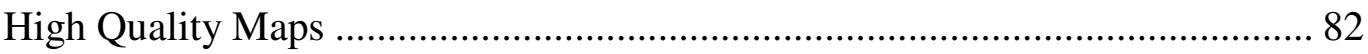

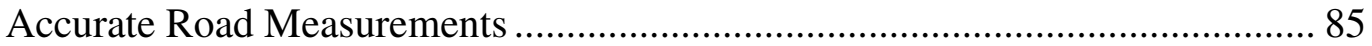

Accurate Stream Channel Measurements ........................................................ 86

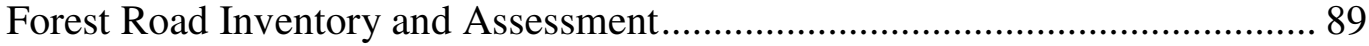

Decision Support for Road Management .................................................... 91

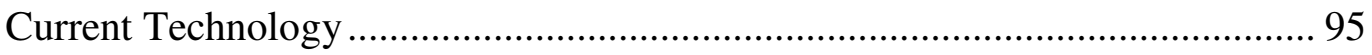

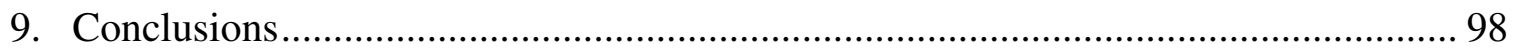

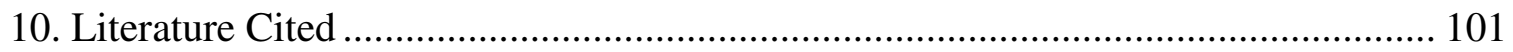

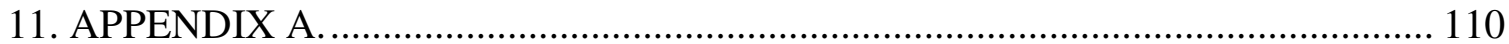




\section{LIST OF TABLES}

Table 1. Factors influencing the quality of LiDAR terrain data................................ 12

Table 2. Vegetation species associated with the Redwood vegetation series................. 26

Table 3. LiDAR flight and sensor specifications................................................. 35

Table 4. LiDAR data within the Little Creek watershed. .......................................... 37

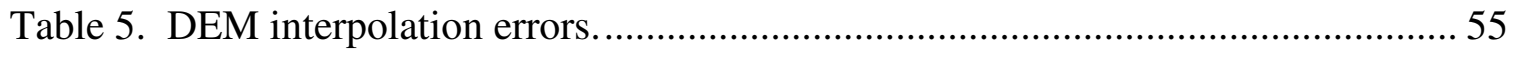

Table 6. Buffer distances for the six study reaches on Little Creek. ............................. 67

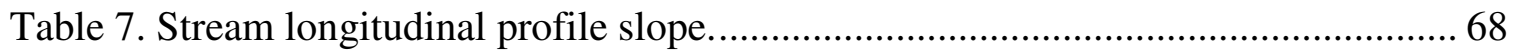

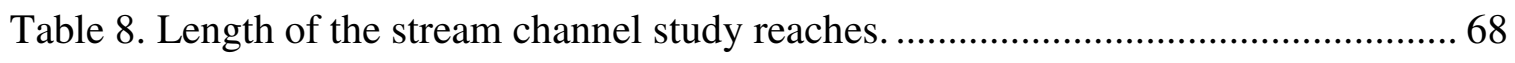

Table 9. LiDAR survey parameters for Swanton LiDAR flights in 2002 and 2008. ...... 95

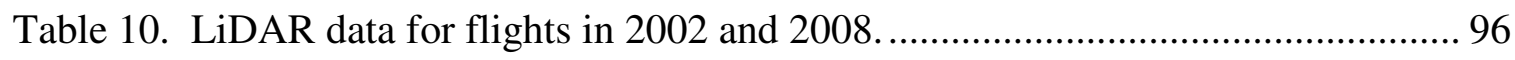




\section{LIST OF FIGURES}

Figure 1. Shaded relief from a 10 m USGS DEM. .................................................. 10

Figure 2. Shaded relief from a $1 \mathrm{~m} \mathrm{LiDAR-derived} \mathrm{DEM.} \mathrm{......................................} 10$

Figure 3. LiDAR vertical error and terrain slope................................................. 13

Figure 4. The Little Creek watershed, tributary to the Scotts Creek watershed............. 25

Figure 5. Little Creek road with a $2.5 \mathrm{~m}$ traveled surface width ................................. 28

Figure 6. Little Creek haul road across low-relief topography .................................. 29

Figure 7. Sample cross section at the Main Stem of Little Creek................................. 30

Figure 8. Channel conditions at the Main Stem of Little Creek.................................. 30

Figure 9. Example of road edges and centerline from ground-based survey .................. 32

Figure 10. Study reaches in the Little Creek watershed. ........................................... 34

Figure 11. Density and distribution of LiDAR ground returns in a sample area............ 36

Figure 12. Distribution of LiDAR point spacing................................................ 38

Figure 13. Watershed area classified by ground point spacing. ................................. 39

Figure 14. Steps toward digitizing forest road features from LiDAR data..................... 42

Figure 15. Example of the digitized road centerline and digitizing cursor.................... 44

Figure 16. Roads and trail features digitized from LiDAR data................................ 45

Figure 17. Little Creek road segment with buffer analysis...................................... 50

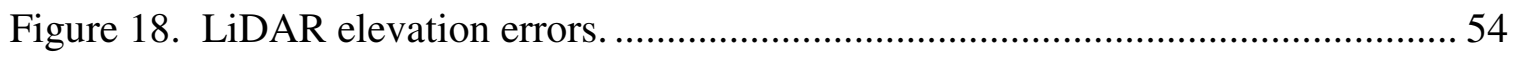

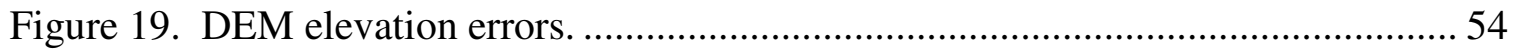

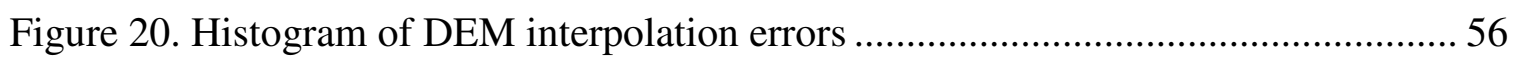

Figure 21. Interpolation errors within the Little Creek watershed............................... 57

Figure 22. A gap in LiDAR ground returns....................................................... 59 
Figure 23. Examples of well defined and poorly defined road segments

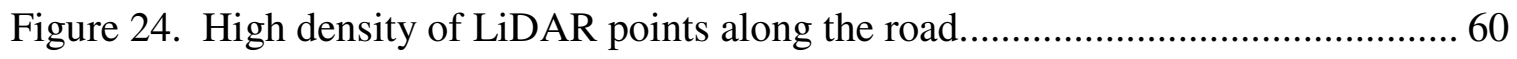

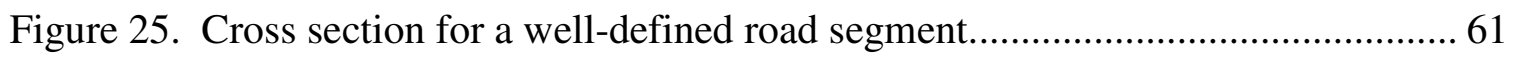

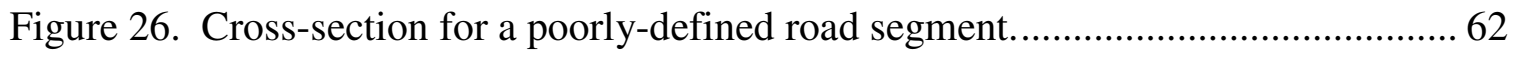

Figure 27. Positional accuracy assessment for the Little Creek road. ............................ 63

Figure 28. Visual agreement of ground survey and LiDAR-derived road profiles......... 64

Figure 29. Detail of Little Creek road longitudinal profile........................................ 65

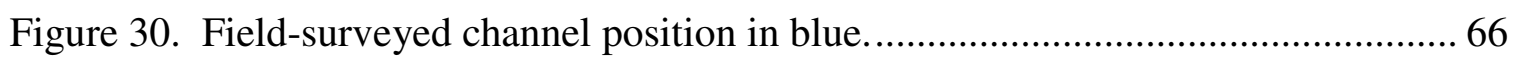

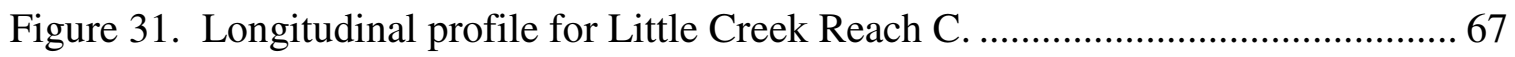

Figure 32. DEM constructed without breakline..................................................... 75

Figure 33. Hypothetical cross-section of the smallest detectable road feature................ 76

Figure 34. Road centerline from field survey (black) and digitized from DEM (red).... 79

Figure 35. Map data from a traditional USGS 1: 24000 topo quad.............................. 84

Figure 36. LiDAR-derived stream channel pattern over a $1 \mathrm{~m}$ color orthophoto............ 88

Figure 37. Hillslope, road cross-section and stream channel................................... 92

Figure 38. Longitudinal profile indicating fill material at road crossing...................... 93

Figure 39. Road fill volume estimated from stream profile and cross section data. ...... 94

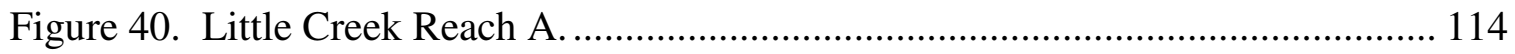

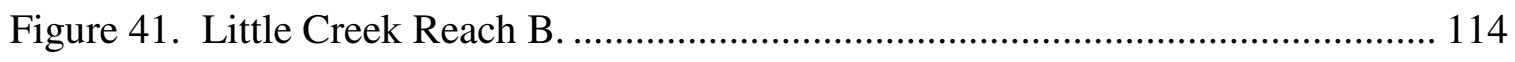

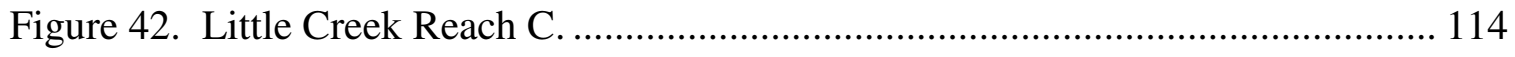

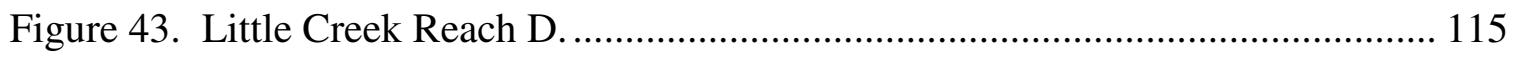

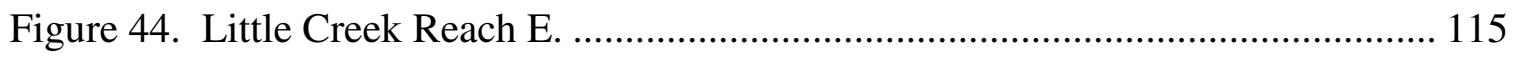

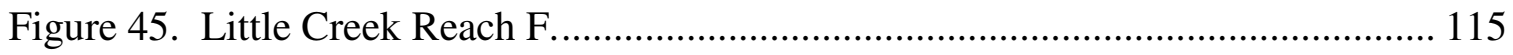


Figure 46. LiDAR-derived road cross-section at outsloped road segment................... 117

Figure 47. LiDAR-derived road cross-section at an insloped road segment. ............... 117

Figure 48. LiDAR-derived road cross-section at a fill crossing. ............................. 118

Figure 49. LiDAR-derived road cross-section at a throughcut road segment. .............. 118 


\section{CHAPTER 1}

Introduction

Airborne Light Detection and Ranging (LiDAR) is a remote sensing technology used to collect highly accurate measurements of earth surface topography. Also termed airborne laser swath mapping, this technique uses an aircraft-mounted laser rangefinder to scan vast areas of land, producing detailed elevation measurements of surface features such as vegetation, buildings, and the ground surface below. LiDAR surveying is accomplished by combining two primary types of data; distance and angle measurements from the laser rangefinder, and precise positional data from the global positioning system (GPS) and Inertial Navigation Unit (INU). The operating principles of LiDAR are fairly straightforward. First, the laser rangefinder scans the terrain, emitting pulses of infrared light at a high pulse-repetition frequency, up to one hundred thousand pulses per second. These pulses are directed toward the ground by an oscillating mirror to produce a swath of measurement points along the flightline of the aircraft. Each LiDAR pulse is used to measure the distance from the aircraft to the landscape below. Distances are determined by recording the amount of time it takes for each pulse to travel from the aircraft to the ground and back to the aircraft. The speed of the pulse, the speed of light, is multiplied by one half of the total travel time to compute distance. The unique distance and angle measurements from each LiDAR pulse are combined with the precise position of the aircraft to construct a three-dimensional representation of terrain, vegetation, structures, or other features of the surveyed landscape (Shrestha et al., 1999).

LiDAR is distinguished from other methods of surveying or remote sensing by its ability to capture detailed topographic data over large areas, without the need for 
extensive post-processing, as with stereo photogrammetry (Flood and Gutelius, 1997). Within the last thirty years this technique has emerged from the stages of testing and development to become a standard tool for topographic data collection in a variety of environmental science and management applications (Flood, 2001). The increasing use of LiDAR since the mid 1990's has been spurred by continued refinement of the technology, increased availability through commercial vendors and improved cost efficiency (Baltsavias, 1999b). Strong demand for LiDAR data has also been driven by the expanding role of Geographic Information Systems (GIS) in resource management and an ever-growing demand for high-quality geospatial information (Carson et al., 2004).

The unique capabilities of LiDAR have sparked particular interest within the field of forest resource management (Reutebuch et al., 2005). With respect to terrain mapping, LiDAR is capable of producing highly accurate Digital Elevation Models (DEMs) of the ground surface, data which could not be widely produced through traditional photogrammetry (Kraus and Pfeifer, 1998). As detailed terrain data has become more accessible, it has been applied toward a growing variety of resource planning and assessment activities. For example, LiDAR terrain data collected over forested areas has been used to map landslides (Schulz, 2007), headwater stream channels (James et al., 2007), identify archeological features (Crow et al., 2007), and plan the alignment of new forest roads (Aruga et al., 2005). While a growing number of natural resources applications have been demonstrated, LiDAR is still regarded as a relatively new technology, and its role in supporting resource management activities remains unclear. Important questions regarding the accuracy and capabilities of this technology 
must still be addressed before LiDAR becomes more broadly adopted into resource management workflows. For example, Can LiDAR elevation data, collected within densely forested areas, replicate time-consuming field-based topographic surveys? What type of topographic features can be reliably identified and measured? And finally, What level of accuracy can be expected from LiDAR in forested terrain?

The broad scope of resource management often requires a wide range of measurements, data, and information to support various decisions. Certainly, requirements for the accuracy and availability of different types of data will vary depending on the application, or the decision at hand. For example, measuring a stream channel longitudinal profile, in order to determine channel gradient, may require a high level of precision. Meanwhile, other field survey activities such as measuring the gradient of forest roads and skid trails, to determine equipment accessibility, or to install suitable drainage features, may not require the same level of precision. The ability for LiDAR terrain data to support various topographic survey activities must be tested in a practical comparison to determine the suitability of LiDAR for resource management.

\section{$\underline{\text { Purpose and Need }}$}

The purpose of this study is to determine the accuracy of LiDAR-derived measurements of forest road and stream channel features located in steep and densely forested terrain. Forest roads play a central role in management activities and also have the potential to adversely impact watershed condition. Unfortunately, forested watersheds often lack a though and up-to-date inventory road features (USFS, 2001). Road inventory data can serve a number of important roles. This data has often been 
used to evaluate past land use impacts, and is also valuable in planning future management activities. For example, the total length and position of forest roads is often used as an index of cumulative watershed effects (McGurk and Fong, 1995). Roads are indeed linked to a number of watershed disturbances and are used as an effective index of watershed disturbance. Road inventory data can again be used also used to identify specific problems areas, such as road crossings, and aids in planning the solution to those problems, such as decommissioning unneeded forest roads (Madej et al., 2006). In each case, a thorough inventory of road features provides a more complete basis for decision making.

Despite the need for comprehensive road inventory datasets, there are few remote sensing methods that can be used to map forest roads. Aerial photo interpretation is often unsuitable for mapping roads simply because dense overstory canopy can obscure these features from view. In contrast with other remote sensing methods, LiDAR has a unique ability to penetrate dense vegetation, mapping the terrain below. Given a sufficiently detailed map of forested terrain, a more thorough inventory of road features may be produced using LiDAR terrain data. Mapping roads using a remote sensing approach versus a ground-based approach offers the ability to map roads efficiently across a large landscape. In this case, roads can be mapped despite difficult terrain conditions or other access restrictions, such as property boundaries. High resolution terrain data also provides the means to accurately map stream channel features across the landscape.

Accurate measurements of stream channel characteristics are commonly used to describe channel form, understand fluvial processes, and assess channel conditions. Detailed field surveys of channel cross-sectional and longitudinal profiles are often 
required to characterize channel topography (Keim et al., 1999). Quantitative descriptions of channel form, such as width-to-depth ratio, entrenchment ratio, slope, and sinuosity, are drawn from these surveys and used to describe channel condition and hydrologic function (Rosgen, 1994). In steep terrain and dense vegetation, these field surveys can be difficult, time consuming, and expensive. Limited time and resources can limit the scale and the number of field survey locations, making it difficult to capture the variety of channel conditions that may exist throughout a given watershed.

The use of LiDAR to measure stream channel characteristics has generated considerable interest among watershed scientists and resource professionals (Snyder and Schultz, 2005). However, this approach must be tested throughout a range of environmental conditions to determine if LiDAR-derived measurements are sufficiently accurate and reliable given different terrain and vegetation conditions. Delineating the location of small stream channels in densely forested and steep terrain, provides a suitable assessment of LiDAR capabilities since these conditions impose significant challenges to any remote sensing technique. Results from this study are intended to help resource managers formulate suitable expectations of LiDAR data collected in forested environments.

Reporting the accuracy of LiDAR-derived features is important for two reasons. First, measuring the accuracy of LiDAR-derived attributes relative to traditional fieldbased techniques will indicate if LiDAR terrain data can support or replicate field-based survey methods. Second, LiDAR-derived features such as digitized roads or stream channels are likely to be incorporated into a GIS for use in forest management, watershed assessments, or hydrologic modeling. Oftentimes the completeness and accuracy of these 
input data are not adequately considered. The potential errors or uncertainty of LiDARderived GIS data should be quantified and acknowledged when used in any decisionmaking process.

\section{$\underline{\text { Objectives }}$}

The primary objectives of this study were to measure forest road and stream channel characteristics using LiDAR terrain data and to determine the accuracy of these measurements relative to field-based survey methods.

Objective I - Determine the completeness and accuracy of the LiDAR-derived forest road measurements.

i. Completeness - Given a ground survey of road position and length, determine the percentage of road length that could be identified and mapped using LiDAR terrain data.

ii. Horizontal error - Determine the $95^{\text {th }}$ percentile horizontal distance separating the LiDAR-derived road centerline and the ground-surveyed centerline.

iii. Road slope error - Determine the difference in road slope measurements obtained using both LiDAR elevations and ground-survey elevations.

iv. Road length - Determine the difference in road length between the LiDARderived road centerline and ground-surveyed centerline. 
Objective II - Determine the accuracy of LiDAR-derived stream channel characteristics.

i. Horizontal error - Determine the $95^{\text {th }}$ percentile horizontal distance separating the LiDAR-derived channel centerline and the ground-surveyed stream centerline.

ii. Stream slope error - Determine the mean difference in channel slope measured with the LiDAR-derived longitudinal profile and the groundsurveyed longitudinal profile.

iii. Stream length - Determine the mean difference in channel length between LiDAR-derived channel centerline and the ground-surveyed channel centerline. 


\section{CHAPTER 2}

Literature Review

\section{$\underline{\text { LiDAR in Forested Environments }}$}

The expanding potential for the use of LiDAR in forest management is well documented in the scientific literature (Bater, 2008; W. W. Carson et al., 2004; Lefsky et al., 2002; Lim et al., 2003; Renslow, 2000). In many examples, LiDAR is used to measure biophysical attributes of the forest stand, such as canopy cover (Coops et al., 2007), tree height (Andersen et al., 2006), stand density, basal area, and volume (Naesset, 2004; Reutebuch et al., 2005). As LiDAR research comes of age, time-series datasets of multiple LiDAR surveys are being used to detect changes and measure forest growth (Woodget et al., 2007; Yu et al., 2004). The use of LiDAR for measuring above ground vegetation attributes is a tremendously active and valuable area of research, though it is often conducted separately from the study of forest terrain and the development of the ground surface DEM. Nevertheless, using LiDAR to obtain accurate vegetation heights requires a high-quality DEM to represent ground surface elevations (Bater, 2008). Therefore, producing accurate ground surface DEMs is critical for a wide range of LiDAR applications. Considering that both the forest canopy and the underlying terrain can be characterized in a single LiDAR survey, one can appreciate the immense amount of information that is provided by LiDAR remote sensing.

In order to recognize the advancements offered with LiDAR terrain mapping, it is helpful to consider the characteristics of conventional digital terrain data. The USGS National Elevation Dataset is composed of the highest quality digital elevation data available throughout the United States, and has been compiled into a seamless raster 
format (http://ned.usgs.gov/). The quality and accessibility of this data makes it indispensible for broad-scale planning and topographic analysis (Pike, 2002). Currently, the highest resolution data available at a national level are the USGS $10 \mathrm{~m}$ and $30 \mathrm{~m}$ digital elevation models. The accuracy of a Level I USGS DEM is defined by the USGS Standards for Digital Elevation Models, which states the following:

A vertical RMSE of 7 meters or less is the desired accuracy standard. A RMSE of 15 meters is the maximum permitted. A 7.5-minute DEM at this level has $a[n]$ absolute elevation error tolerance of 50 meters (approximately three times the 15-meter RMSE) for blunders for any grid node when compared to the true elevation. (USGS, 1992)

While these products are valuable for broad-scale planning and analysis, the coarse resolution and vertical error are not suitable fine-scale or site-specific topographic mapping. To further quantify the vertical error of these products, the accuracy of 23 USGS DEMs from forested areas of Oregon and Washington were evaluated using 4,355 photogrammetric checkpoints (Carson and Reutebuch, 1997). An average RMSE of 7 meters was achieved across the 23 DEMs, however accuracy ranged significantly among individual DEMs from as low as $2 \mathrm{~m}$ up to $14 \mathrm{~m}$ RMSE.

Today, airborne LiDAR surveys conducted over a forested landscapes can yield highly-accurate, high-resolution digital elevation models. LiDAR-derived DEMs are commonly produced at a resolution of $1 \mathrm{~m}$ to $3 \mathrm{~m}$ and have been evaluated with a vertical error ranging from 0.57 m RMSE (Kraus and Pfeifer, 1998), to 0.28 m RMSE (Kobler et al., 2007). This combination of high resolution and improved vertical accuracy provides a much more detailed representation of forested terrain. The difference in topographic 
detail provided by a conventional USGS $10 \mathrm{~m}$ DEM and a LiDAR-derived $1 \mathrm{~m}$ DEM is presented in Figures 1 and 2 below.

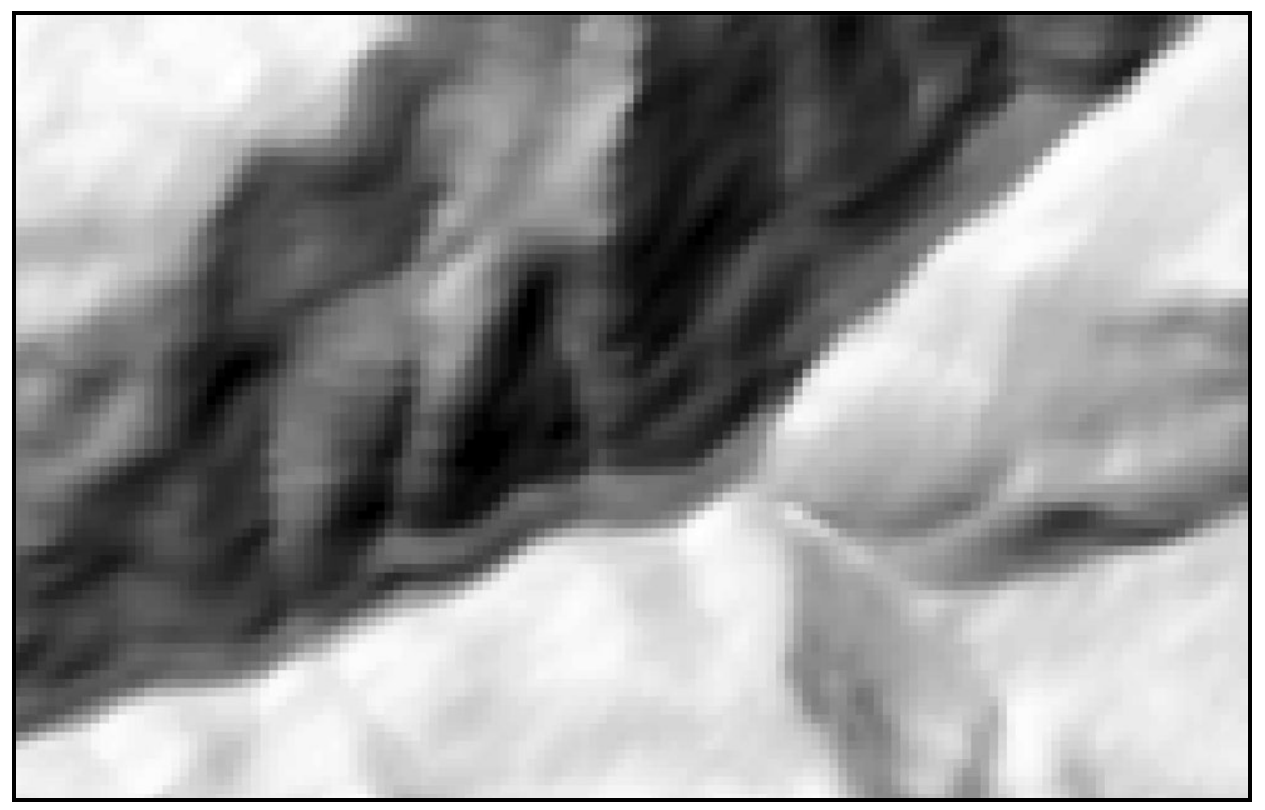

Figure 1. Shaded relief from a $10 \mathrm{~m}$ USGS DEM. Portion of the Little Creek watershed near Davenport, CA.

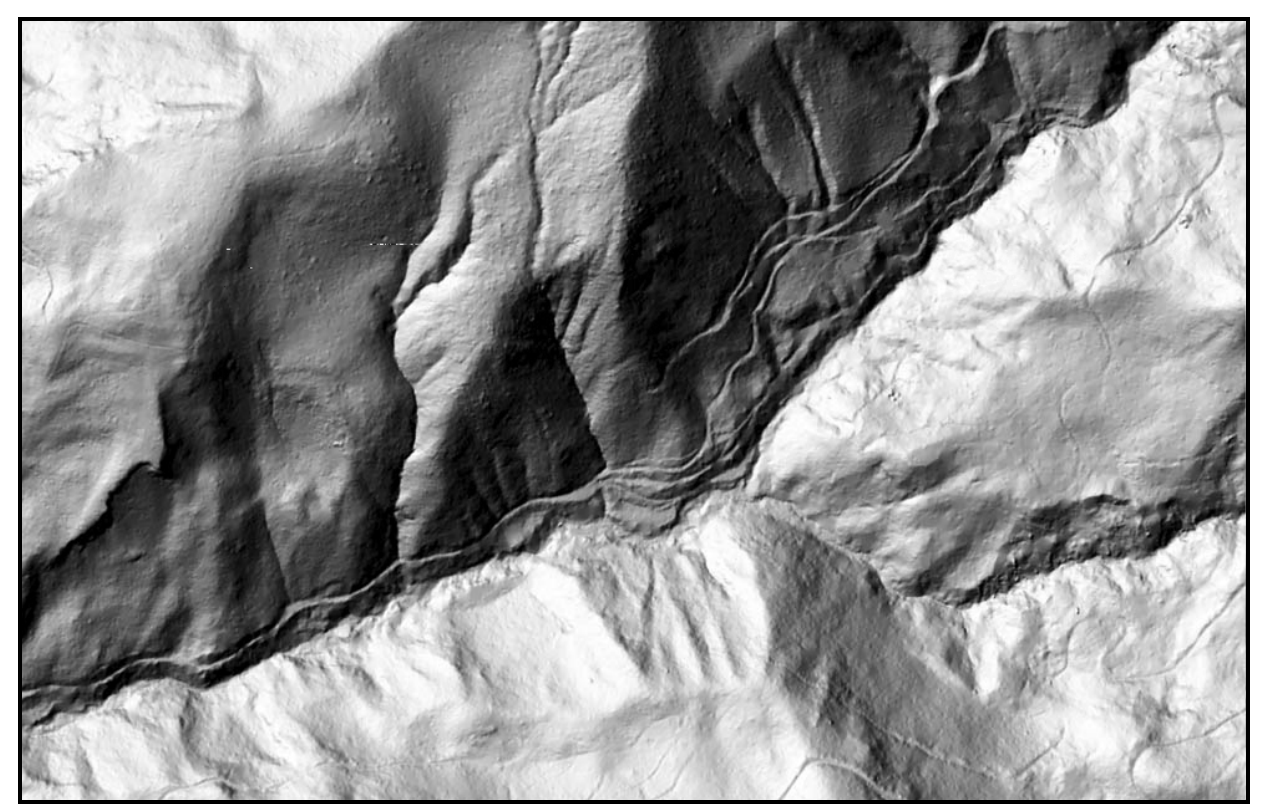

Figure 2. Shaded relief from a $1 \mathrm{~m}$ LiDAR-derived DEM. Portion of the Little Creek watershed near Davenport, CA. 


\section{$\underline{\text { LiDAR Accuracy }}$}

The improved accuracy of LiDAR-derived DEMs has prompted their use in a variety of topographic mapping applications. Increasingly, these DEMs are used for applications that require high level of vertical and positional accuracy, such as in preliminary forest engineering and forest road design (Krogstad and Schiess, 2004). To ensure that a LiDAR-based approach can provide sufficient accuracy for a given application, the quality of LiDAR data must be carefully evaluated. It is therefore important to understand the basic principles of LiDAR data collection and processing. Three phases of LiDAR data production are outlined in Table 1; acquisition, postprocessing, and end-use. In each of these phases, there are different factors that influence the quality of the data. Since there are such a wide variety of system configurations and landscapes over which LiDAR surveys are conducted, it is difficult to anticipate the results that a particular LiDAR survey may provide. In short, no two LiDAR surveys are exactly alike and therefore, both the data and survey parameters should be inspected.

LiDAR data collected in forested environments requires especially careful consideration. The commonly reported vertical accuracy for LiDAR data is $0.15 \mathrm{~m}$ RMSE. This accuracy is typically evaluated in optimum conditions where LiDAR pulses are reflected from hard, flat surfaces with low slope and with no interference from surface features like vegetation (Baltsavias, 1999b). It is not often recognized that the quality of LiDAR elevations can vary dramatically across the landscape as a function of terrain steepness, vegetation density, and other factors. 
Table 1. Factors influencing the quality of LiDAR terrain data.

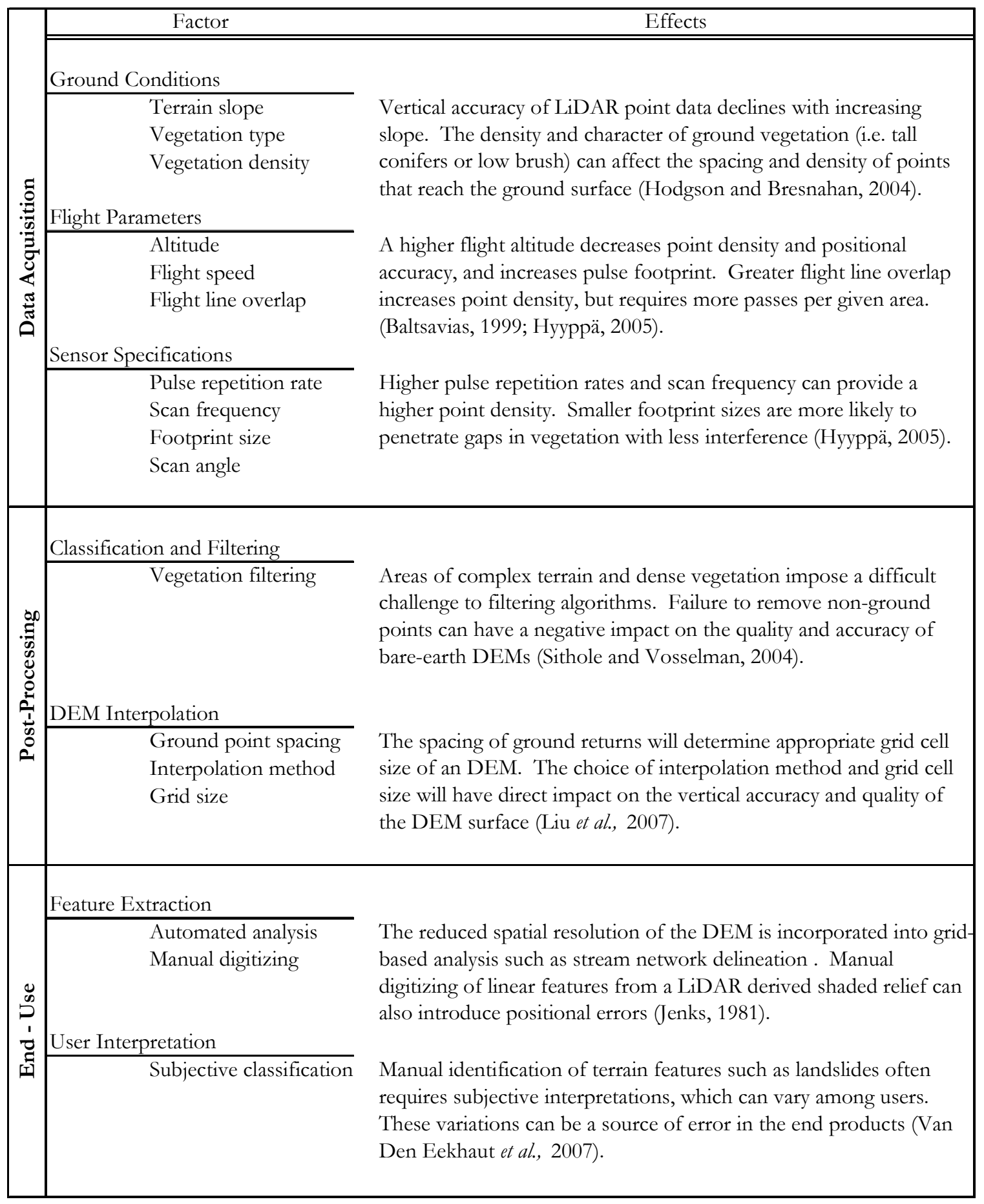


The accuracy of LiDAR data in forested environments has been investigated in a number of empirical studies (J. Hyyppä et al., 2000; Kraus and Pfeifer, 1998; Reutebuch et al., 2003). These studies typically compare LiDAR-derived elevations to elevations obtained through a ground-based survey, as with a total station. Ground-survey checkpoints located in varying terrain and vegetation conditions are used to assess variations LiDAR error. For example, LiDAR data evaluated on slopes ranging from 5\% to $60 \%$ (Figure 3 ) demonstrated errors that increased from $0.15 \mathrm{~m}$ to $0.80 \mathrm{~m}$ RMSE (Kraus and Pfeifer, 1998).

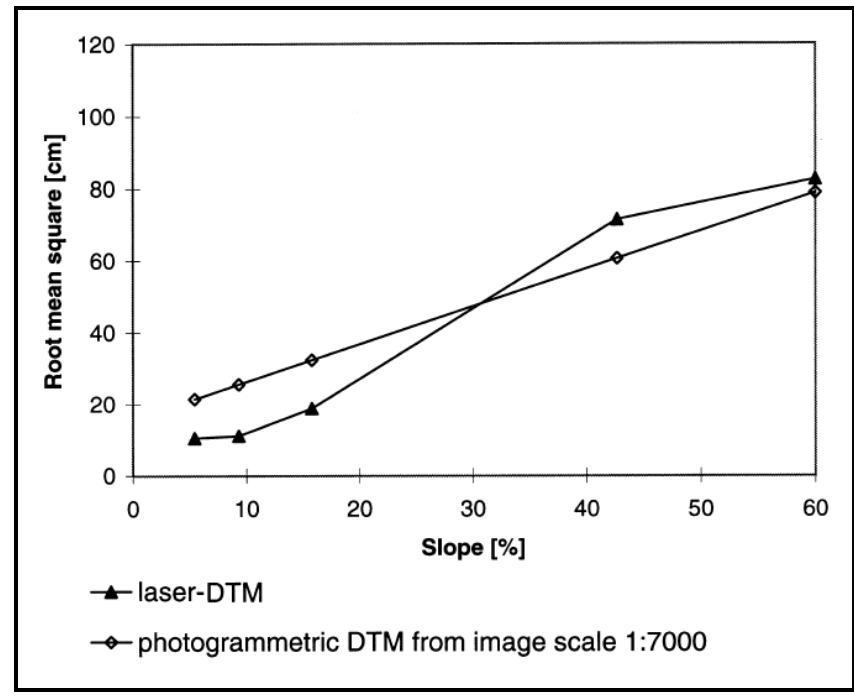

Figure 3. LiDAR vertical error and terrain slope (Kraus and Pfeifer, 1998).

Similar results are reported by Hyyppä et al., (2000), where a RMSE of 0.15 m was achieved in flat terrain, but exceeded $0.40 \mathrm{~m}$ for terrain slopes greater than forty percent. On gentle slopes, ranging up to 17\% Hodgson and Bresnahan (2004) reported little evidence for increased elevation errors, though LiDAR-derived slope slightly underpredicted true terrain slope. 
The density and structure of vegetation can also influence the accuracy LiDAR elevation data. The effects of vegetation on LiDAR accuracy was evaluated within stands of mature Douglas-fir on managed forest land in western Washington (Reutebuch et al., 2003). An overall accuracy of $0.32 \mathrm{~m}$ RMSE was determined from a comparison of nearly 350 ground-surveyed checkpoints. A small difference in vertical error was observed for checkpoints located under differing canopy conditions; clearcut $\pm 0.16 \mathrm{~m}$, heavily thinned $\pm 0.18 \mathrm{~m}$, lightly thinned $\pm 0.18 \mathrm{~m}$, and uncut $\pm 0.30 \mathrm{~m}$. The effect of nearground vegetation was also considered. Surprisingly, the additional vertical error for checkpoints located in areas of dense near-ground vegetation compared to open areas were rather small, only $0.11 \mathrm{~m}$ (Reutebuch et al., 2003).

A similar study conducted under a forest canopy dominated by pine, spruce and birch resulted in a DTM with an overall accuracy of $\pm 0.20 \mathrm{~m}$ (H. Hyyppä, 2005). A maximum systematic error of $0.08 \mathrm{~m}$ was observed for points located directly beneath overstory canopy (Hyyppä, 2005). An evaluation of LiDAR error conducted by Hodgson and Bresnahan (2004), point towards a small, though statistically significant difference in LiDAR error across different vegetation classes.

While the influence of vegetation may have a relatively small influence on LiDAR elevation accuracy, this vegetation also affects the density and distribution of laser pulses that reach the ground surface (Hodgson and Bresnahan, 2004; Reutebuch et al., 2003). The effect of vegetation on reducing the density of LiDAR ground points may present a more significant limitation on the quality of LiDAR data in forested areas. Variations in point density and gaps in ground return data are expected to some degree since some individual tree canopies may prevent incoming LiDAR pulses from reaching 
the ground surface. However, the reduced density and irregular spacing in ground returns presents a number of concerns. For areas where a low ground point density is achieved, LiDAR data may not represent the true topography of the ground surface. These areas can be of particular concern if the terrain data is to be used for identifying and mapping subtle topographic features (Reutebuch et al., 2003). Ensuring a dense and welldistributed coverage of LiDAR ground points should be a top concern for the development of a high quality DEM (Liu, 2008).

In order to achieve a dense coverage of ground returns in a forested environment, a number of options can be considered. LiDAR flights are often conducted during "leafoff' conditions, when the seasonal cover from deciduous vegetation is at a minimum $(\mathrm{H}$. Hyyppä, 2005). However, vegetation alone is not the only variable that affects ground point density. LiDAR sensor specifications and flight parameters are also adjusted to produce a targeted point density. In general, higher pulse repetition rate, lower flight altitude, slower flight speed, and greater flight line overlap will increase point density, and should also increase the number of points reaching the ground. Lower flight altitude is particularly important in reducing the diameter of the laser footprint as it reaches the ground (Hyyppä, 2005). A laser pulse with a smaller diameter is more likely to reach the ground surface without interference from tree canopies or vegetation (Hodgson and Bresnahan, 2004; Hyyppä, 2005). However, lower flight altitudes and greater flight line overlap will require a greater number of passes per given area, and will increase the cost of the survey.

As presented in Table 1, a number of factors contribute to the accuracy of LiDAR in forested environments. The influence of these factors continues to play a role during 
the filtering or classification of LiDAR returns and in the interpolation of the digital elevation model. Despite the technical challenges, LiDAR has gained acceptance as an excellent source of topographic data that can serve a wide variety natural resources applications.

\section{Applications for Watershed Management and Assessment}

Topography has a fundamental influence on hydrologic, geomorphic and land-use patterns. Characteristics of topography such as slope steepness, aspect, curvature, and contributing area are primary controls on many hillslope and fluvial processes (McKean and Roering, 2005). In this way, topography also has a strong influence on management decisions. Understanding the links between topography, geomorphic processes, and management activities is a crucial component of resource management. Many best management practices (BMPs) in forestry operations include considerations regarding terrain and geomorphic conditions. For example, the requirements for a Timber Harvest Plan (THP) submitted in the state of California specify that a Registered Professional Forester (RPF) shall conduct a field evaluation to identify and map areas that may be adversely impacted by proposed management activities. Terrain conditions that are commonly identified include: unstable and erodible watercourse banks, unstable upslope areas, overflow channels, and flood prone areas. Furthermore, these conditions must be mapped with sufficient detail to permit effective communication among review team representatives (CDF, 2007). Mapping these terrain conditions and communicating the underlying geomorphic processes require a thorough understanding and detailed depiction of topography. Here, LiDAR terrain data offers resource managers a vastly 
improved ability to visualize and communicate topographic conditions, both among professionals, and with the public. The advantage of LiDAR data is that it is both highly detailed and sufficiently broad in extent to serve site specific projects, over large-scale forestry and watershed management operations.

The identification and measurement of landslides in forested environments has been particularly aided by using LiDAR technology (McKean and Roering, 2005; Metternicht et al., 2005; Glenn et al., 2006; Van Den Eeckhaut et al., 2007; Schulz, 2007). In these studies, landslides were visually identified using GIS, and DEM derivatives such as shaded relief maps, slope grids, contour lines, and topographic profiles. Leveraging DEM data to produce these GIS products has improved landslide inventory maps, allowing geologists to identify more landslide features than previously documented (Van Den Eeckhaut et al., 2007; Schulz, 2007). In particular, LiDAR has enabled geologists to overcome the limitations of mapping landslides through aerial photo interpretation in forested environments. Compared to detailed ground surveys, aerial photo interpretation in forested areas has been shown to detect for as little as $15 \%$ to $30 \%$ of landslide features, accounting for $50 \%$ to $70 \%$ of landslide erosion volume (Brardinoni et al., 2003; Robison et al., 1999).

LiDAR-derived landslide inventory maps have also been compared to field-based landslide maps for forested landscapes. In a study by Van Den Eeckhaut et. al, (2007), a panel of seven expert geomorphologists created independent landslide maps based on LiDAR-derived products from a $5 \mathrm{~m}$ DEM. Results indicate that $70 \%$ of landslides identified in the field were mapped through LiDAR image interpretation. Furthermore, by using the LiDAR interpretation from the seven experts along with field investigation, 
it was possible to create a high quality landslide inventory map with the following findings: $76 \%$ of the landslides mapped in the field were mapped using the LiDARderived images, $13 \%$ of landslides incorrectly mapped in the field could be corrected with the LIDAR images; and $11 \%$ of landslides were identified only through the use of LIDAR-derived images (Van Den Eeckhaut et al., 2007). Similar to the task of identifying landslides, mapping roads in forested areas, an objective of this study, is also limited when using traditional remote sensing methods.

\section{Applications for Mapping Forest Roads}

Acquiring and updating road inventory information for GIS applications can be a costly and time consuming endeavor (Jazouli et al., 1994). In response to this challenge, tremendous research has been directed towards automated or semi-automated techniques to extract road network information from remote sensing data (Mena, 2003; Saleh, 2004). Aerial photographs and satellite imagery are the most common data sources for such techniques, though airborne LiDAR data has also been used (Clode et al., 2004; Clode et al., 2007; Opitz et al., 2006). With the use of LiDAR data, additional road attributes, such as road grade, cross-sectional slope and road prism geometry can also be extracted. Three-dimensional attributes of the road and nearby terrain can be used to assess the condition and safety of road segments. For example, airborne LiDAR data has been used to measure line-of-sight visibility and stopping distance for horizontal and vertical road curves (Shamayleh and Khattak, 2003). Road slope data was also used to model precipitation runoff from road surfaces and to evaluate slope-length limitations for heavy trucks (Souleyrette et al., 2003). 
While these applications exist for road networks in open areas, there are relatively few publications that describe mapping roads in forested landscapes, even through manual photo interpretation (Asner et al., 2002; Jazouli et al., 1994). Traditional remote sensing data are often limited by coarse resolution and the inability to penetrate dense canopy. For example, analysis of $30 \mathrm{~m}$ resolution National Land-Cover Data in the Pacific Northwest detected less than $40 \%$ of road-related forest fragmentation compared to the same analysis conducted with a detailed national road and street map (Riitters et al., 2004). When photo interpretation is inadequate, road data may be obtained through field mapping with a combination of GPS and GIS. However, even field-based surveys may not guarantee a complete road map due to access limitations, unknown road locations and the limitations of GPS signal reception. There are few published assessments of the accuracy or completeness of forest road data. In one case study, the completeness of a forest road database was evaluated in order to model the effects of roads at a large-landscape scale. Of the $200,000 \mathrm{~km}$ of inventoried roads it was estimated that $20 \%$ to $50 \%$ of additional road length may have been undocumented (Aitken and Hayes, 2006).

Analysis of LiDAR terrain data presents an opportunity to remotely map forest roads and skid trails located under dense forest canopy, yet this application has received little scientific documentation (Espinoza and Owens, 2007). Rieger et al., (1999), present an effective technique for the semi-automated extraction of forest road locations from LiDAR terrain data. In this method, a very high resolution $0.2 \mathrm{~m} \times 0.2 \mathrm{~m}$ DEM was used to produce a terrain slope grid of the same resolution. Image processing was performed to detect road edges on the grayscale slope grid using an adaptive contour method 
referred to as "snakes" (Laptev et al., 2000). The positional accuracy of the extracted road location was evaluated in comparison to field-based mapping. Major topographic breaklines that defined the road prism were located within one to two meters of their field-measured location, while road width was also accurately depicted (Rieger et al., 1999).

LiDAR terrain data has also been used to plan the alignment of new forest roads (Akay, 2004; Aruga et al., 2005; Krogstad and Schiess, 2004; Sessions et al., 2006). High-resolution terrain data was used in conjunction with a computerized route projection program to simultaneously determine the optimal horizontal and vertical road alignment. This route can be optimized with respect to minimizing the volume of cut and fill, attaining a specified road grade, or accommodating specific road drainage structures. The high-resolution DEM was also used to model sediment and runoff contribution of different road design alternatives. Together these applications can provide optimized design alternatives to decision-makers, and potentially reduce the costs and design time of new road construction.

Some limitations on the use of LiDAR data for engineered forest road designs have also been recognized (Krogstad and Schiess, 2004). First, areas that receive low LiDAR point penetration may not convey detailed terrain information. It is especially important for DEMs users to be aware that the accuracy of the DEM surface can be significantly degraded in these situations. Second, ground surface or subsurface characteristics, such as bedrock outcrops or saturated soils may not be readily identified from the LiDAR terrain data. Identifying these conditions is critical to choosing a suitable road alignment, and therefore a LiDAR-derived road alignment still warrants 
field verification. Despite these limitations, LiDAR terrain data often provides a high quality source of topographic data, which can improve the efficiency and reliability of initial road design (Krogstad and Schiess, 2004).

\section{Applications in Hydrology and Geomorphology}

High-resolution topographic mapping of stream channels has received widespread interest in the scientific literature. Relatively early LiDAR research demonstrated the use of LiDAR to measure entrenched stream channel and gully features in the semiarid southwest (Ritchie, 1996). Stream channel and gully cross-sections for features measuring $10 \mathrm{~m}$ to $25 \mathrm{~m}$ in width and $2 \mathrm{~m}$ to $3 \mathrm{~m}$ in depth were extracted from LiDAR ground returns, though no comparison was made to field-based measurements. More recently, the capabilities and limitations of LiDAR-derived measurements of channel morphology are described in several filed-based evaluations (Bowen and Waltermire, 2002; James et al., 2007; Jones et al., in press; Miller et al., 2004). Obtaining a suitable description of channel morphology requires a sufficient density of LiDAR points data in order to characterize channel banks, terraces and other topographic features. In this way, LiDAR terrain data may have a limited ability to characterize the cross-sectional geometry for small headwater streams and gullies (James et al., 2007).

For areas with smaller stream channels, LiDAR terrain data is often used to delineate channel networks (Colson, 2006; Garcia, 2004; Murphy et al., 2007), obtain longitudinal profiles (Vianello et al., 2009), identify barriers to fish migration or locate the channel initiation point (Mouton, 2005). In these instances, high-resolution DEM data are used in conjunction with grid-based flow accumulation algorithms such as Arc 
Hydro (ESRI, 2006a) or TauDEM (Tarboton, 1997), which delineate stream channel position based on DEM topography. The use of LiDAR DEMs with these automated processes can offer a dramatic improvement in the accuracy of channel position, length, slope and point of initiation when compared to the USGS 10 m DEM (Mouton, 2005; Snyder and Schultz, 2005). In a study by Colson et al. (2006) the positional accuracy of stream channel locations derived from a $6 \mathrm{~m}$ DEM was evaluated in a four-county region of North Carolina. Forty percent of the measured channel locations were located within 3 meters of the GPS surveyed channel location. The accuracy of these channel locations was a dramatic improvement over traditional sources of stream line data (Colson, 2006).

Beyond the basic mapping and measurement of topographic features, LiDARderived DEMs offer improvements to a multitude of large-scale fluvial and hydrologic modeling applications (Charlton et al., 2003; Jones et al., in press; Nelson et al., 2006; Pereira and Wicherson, 1999). The refinement in topographic accuracy and resolution can also improve physically-based models, such as landslide hazard predictions (Tarolli and Tarboton, 2006) or estimates of soil moisture across the forested landscape (Tenenbaum et al., 2006).

LiDAR surveying has been applied to a variety of natural resources measurement activities. As this research has matured, the capabilities of LiDAR have been evaluated within various environmental settings. Ground conditions such as steep terrain slope and dense vegetation can impose greater uncertainty in LiDAR elevation data. The accuracy of end products such as a DEM should be carefully evaluated with respect to these ground conditions. Applications requiring a high level of accuracy and resolution such as engineering designs of forest roads or mapping stream channel cross-sections may be 
especially sensitive to the limitations in LiDAR accuracy or ground coverage. A full understanding of the acquisition and post-processing of LiDAR data will help to determine the suitability of LiDAR data to meet rigorous accuracy requirements. Despite the difficult conditions imposed by forested environments, LiDAR terrain data is often more detailed and accurate and than traditional remote sensing techniques, while covering areas that would be too large for practical field-based surveying. 


\section{CHAPTER 3}

Study Location and Site Description

The geographic setting for this study is the Santa Cruz Mountains on California's Central Coast. The study site is located $19 \mathrm{~km}$ north of the City of Santa Cruz in the Little Creek watershed, a 526-hectare tributary watershed to the Scotts Creek watershed (Figure 4). The lower portion of the Little Creek drainage lies within Swanton Pacific Ranch, a 1,320-hectare educational and research facility owned by the California Polytechnic State University Corporation and managed by the College of Agriculture, Food, and Environmental Sciences. Topography within the Little Creek watershed is steep and rugged, with elevations ranging from $12 \mathrm{~m}$ to $488 \mathrm{~m}$ and an average ground surface slope of 45 percent. The watershed contains approximately $7 \mathrm{~km}$ of streams including first and second-order streams as defined by Strahler (1964), based on the USGS 1: 24,000 topographic quadrangle.

Overstory forest canopy in the Little Creek watershed is dominated by secondgrowth coast redwood Sequoia sempervirens (D. Don) Endl., but also includes components of Douglas-fir Pseudotsuga menziesii (Mirbel) Franco var. menziesii and Tanoak Lithocarpus densiflorus (Hook. \& Arn.) Rehder. Mature red alder Alnus rubra Bong. are a dominant component of the riparian vegetation community and are among the few deciduous overstory species in the watershed. Overstory canopy cover has been measured using a vertical densitometer at thirty forest inventory plots throughout Little Creek. Percentage canopy cover from these measurements ranged between forty to 
ninety six percent, with an average of eighty percent (Swanton Pacific Ranch, 2009 unpublished data).

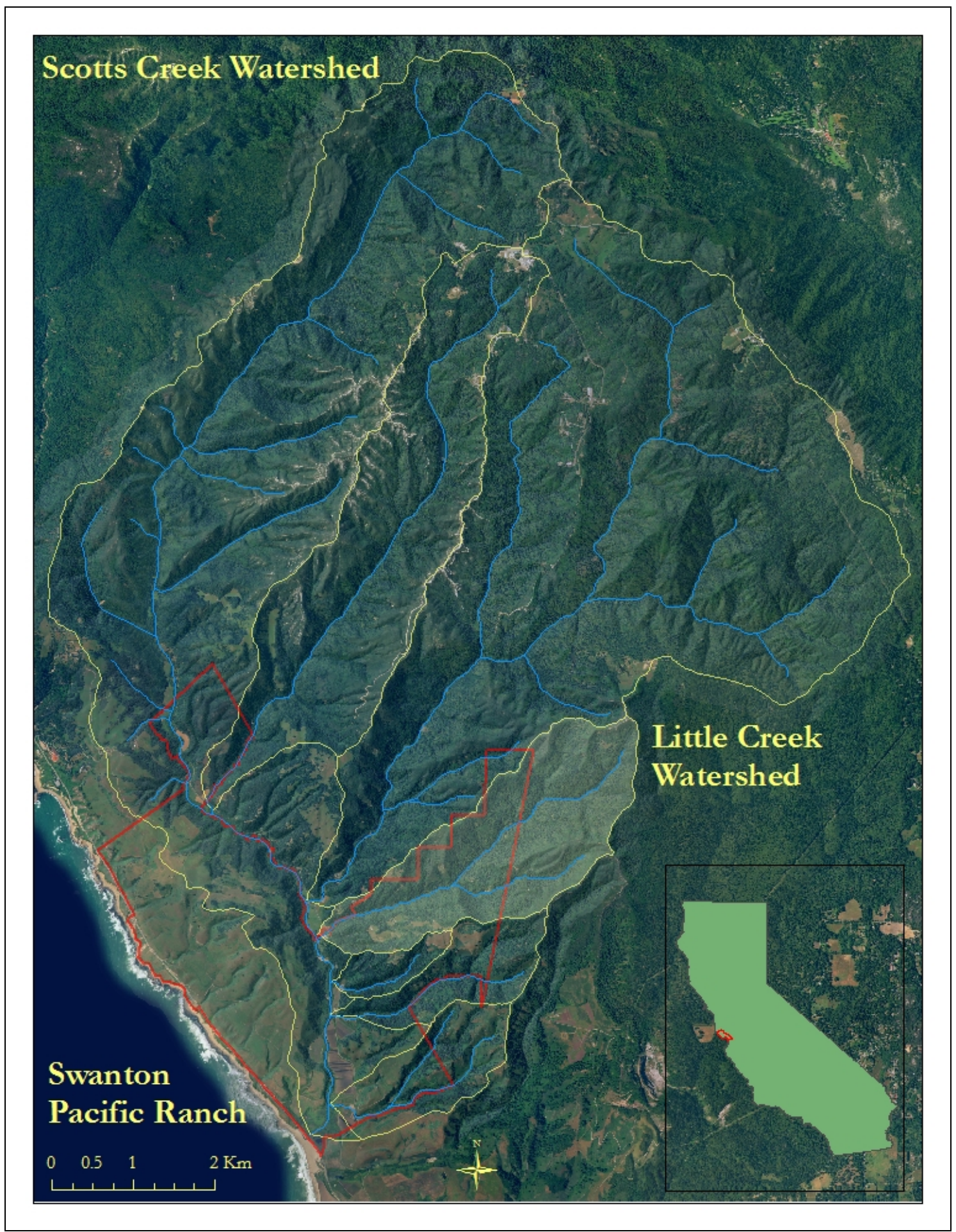

Figure 4. The Little Creek watershed, tributary to the Scotts Creek watershed. Swanton Pacific Ranch property boundary in red. 
Common trees and understory plant species associated with the redwood forest type is included in Table 2, though the local diversity of plant species is known to be much greater. An additional survey of plants native to the Scotts Creek Watershed indicates over 120 species can be identified along the Little Creek road (West, 2009). Understory species found in Little Creek include black berry Rubus ursinus, thimbleberry rubus parviflorus (Nutt.), elder berry Sambucus nigra (L.) ssp. canadesis (L.) R.Bolli , stinging nettle Urtica dioica (L.), elk clover Aralia californica S. Watson, and poison oak Toxicodendron diversilobum (Torr. \& A.Gray) Greene.

Table 2. Vegetation species associated with the Redwood vegetation series (Gaedeke, 2006; Hickman, 1993; Sawyer and Keeler-Wolf, 1995)

\begin{tabular}{|l|l|}
\hline Latin name and author & Common name \\
\hline \hline Acer macrophyllum Pursh & Bigleaf maple \\
\hline Arbutus menziesii Pursh & Madrone \\
\hline Berberis nervosa Pursh Little & Oregon-grape \\
\hline Blechnum spicant (L.) Sm. & Deer fern \\
\hline Carex globosa Boott & Round-fruited sedge \\
\hline Gaultheria shallon Pursh & Salal \\
\hline Iris douglasiana Herb. & Douglas iris \\
\hline Lithocarpus densiflora (Hook. \& Arn.) & Rehder Tanoak \\
\hline Marah fabaceus (Naudin) & Greene Man root \\
\hline Oxalis oregana Nutt. & Redwood oxalis \\
\hline Polypodium californicum Kaulf. & California polypody \\
\hline Polystichum munitum (Kaulf.) C. & Presl Sword fern \\
\hline Pseudotsuga menziesii (Mirbel) Franco var. menziesii & Douglas-fir \\
\hline Pteridium aquilinum (L.) & Kuhn Bracken \\
\hline Sequoia sempervirens (D. Don) Endl. & Redwood \\
\hline Trillium ovatum (Pursh) & Trillium \\
\hline Umbellularia californica (Hook \& Arn) Nutt. & California bay \\
\hline Vaccinium ovatum Pursh & Black huckleberry \\
\hline Woodwardia fimbriata Sm. & Chain fern \\
\hline
\end{tabular}




\section{The Little Creek Road}

The Little Creek road is a private forest haul road used to access the Little Creek watershed for forest management, field research, and educational activities. Nearly half of the $4 \mathrm{~km}$ haul road follows the alignment of a former railroad grade, which was constructed in the early 1900s. Remaining portions of the road were constructed using more modern practices in the late 1980's. Roads that follow former railroad grades typically have low, consistent slopes and are often located along the base of hillslopes, nearer to stream channels, or on sideslopes, rather than on upper ridges. The continued use and maintenance of old railroad grades is a common practice within the Santa Cruz Mountains due to the prevalence of these features. If these existing grades are stable, these locations are typically retained or reconstructed in favor of new road construction, which carries a high cost and high risk of erosion from newly disturbed soils (Weaver and Hagans, 1994).

Physical characteristics of the watershed and the Little Creek road are consistent with the broader Santa Cruz Mountains, and therefore, provide a suitable setting for this assessment. The typical road surface width varies from $2.5 \mathrm{~m}$ to $3.5 \mathrm{~m}$ (roughly 8 to 12 feet), while the width of the entire road bed may measure 5 meters or more (Figure 5). 


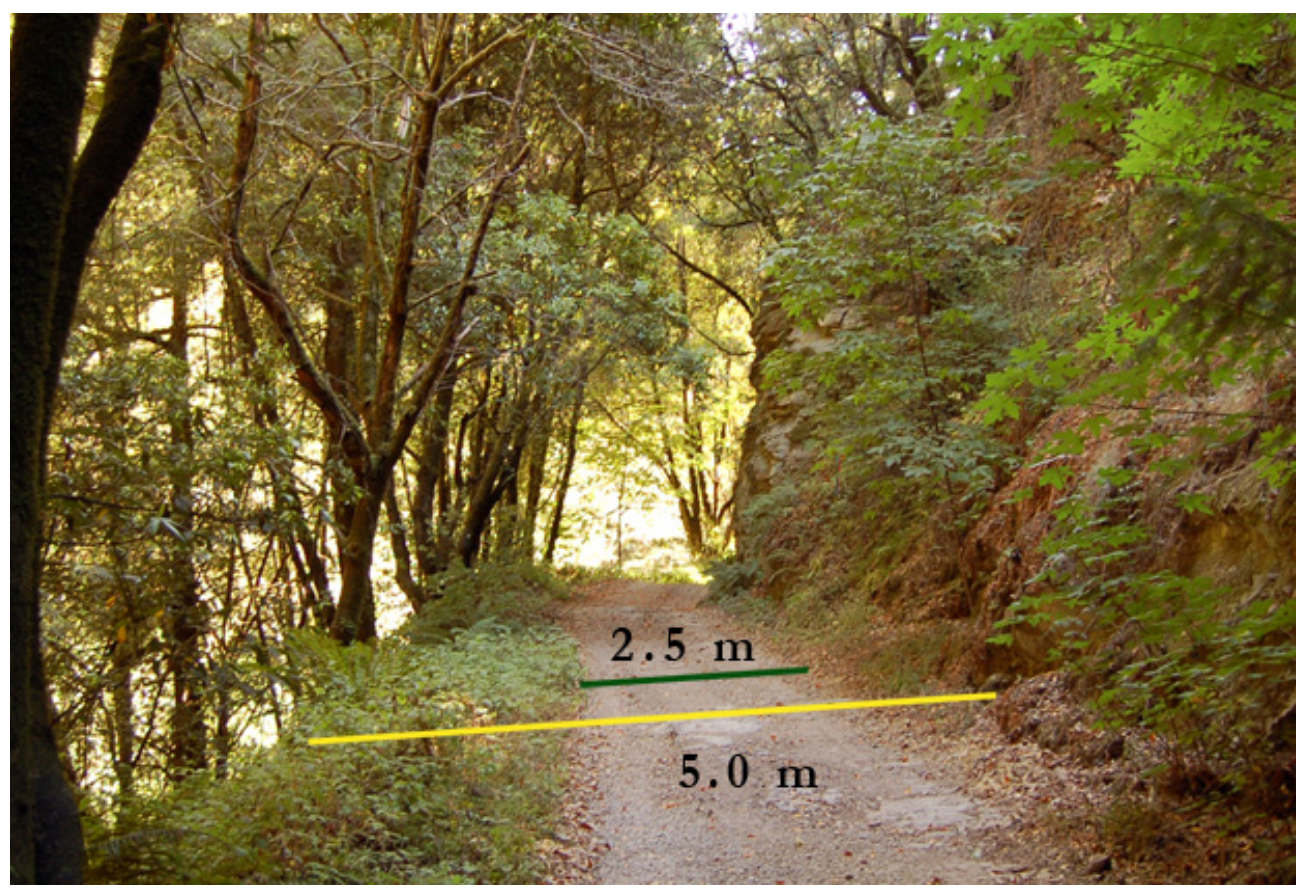

Figure 5. Little Creek road with a $2.5 \mathrm{~m}$ traveled surface width and $5.0 \mathrm{~m}$ road bed width. Note the tall, vertical cutslope in background.

The Little Creek road, as with other roads constructed on steep inner-gorge slopes often requires especially tall and steep cutslopes. Near-vertical cutslopes excavated from the mudstone bedrock (Figure 5), can measure up to 8 meters in height in some locations. As the haul road transitions from the historic railroad grade to more modern locations, a range of road cross-sectional designs are encountered including; full bench cut, balanced cut and fill, full-fill crossings, and throughcut. The last 400 meters of the haul road traverses over an area of low topographic relief where the road has no cut or fill slope and imposes very little disturbance to the surrounding topography (Figure 6). 


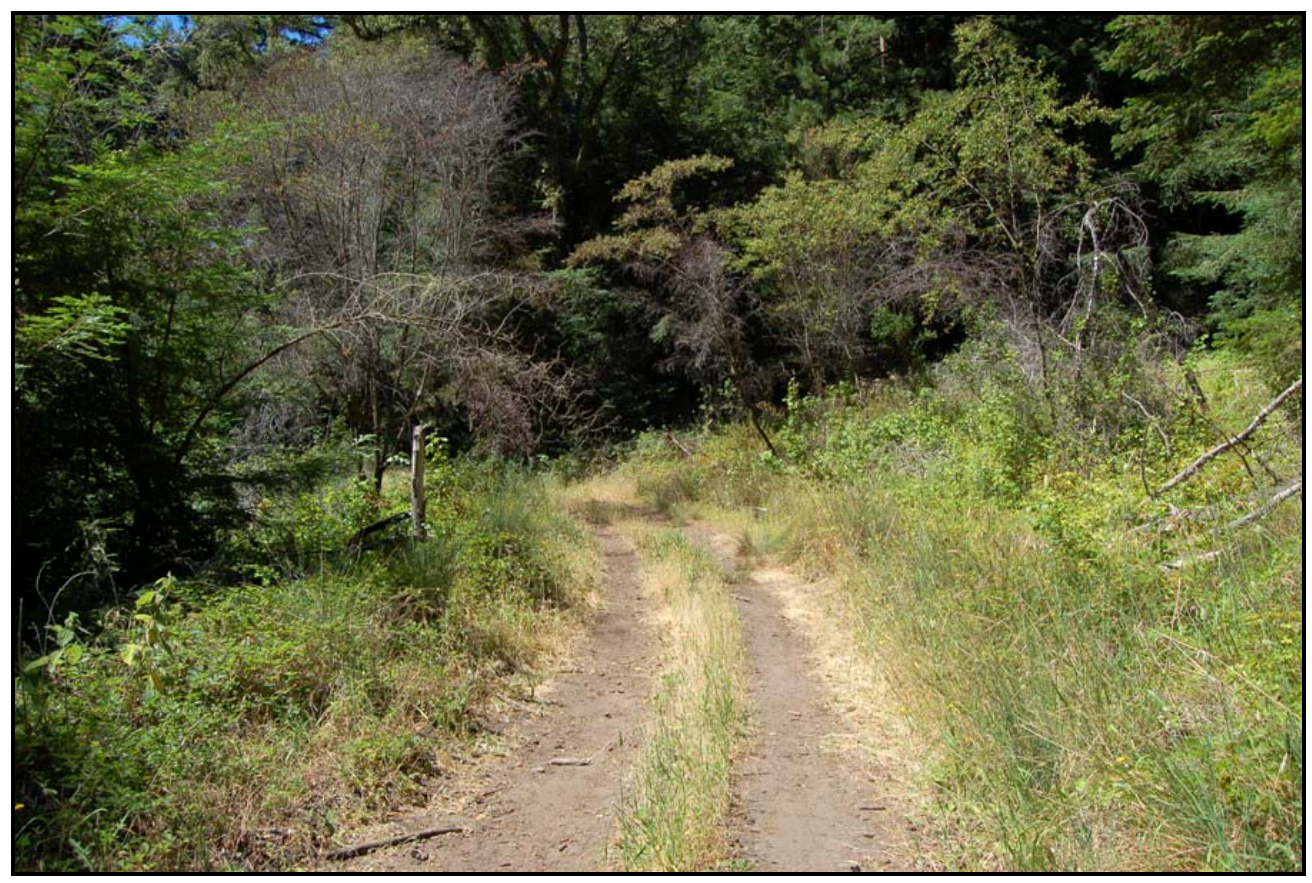

Figure 6. Little Creek haul road across low-relief topography

\section{Little Creek Stream Channel}

The Little Creek stream channel network (Figure 1) is composed of a secondorder main stem reach, which receives flow from the North Fork and South Fork, two first-order tributaries. These channels are characterized by steep longitudinal slopes and highly variable bed morphology. The bankfull channel typically measures 3.5 to 6 meters (12 to $20 \mathrm{ft}$.) in width, while bankfull depth is commonly $0.75 \mathrm{~m}(2.5 \mathrm{ft}$.) or less (Figure 7). The photo in Figure 8 illustrates the channel conditions on the Main Stem of Little Creek. 


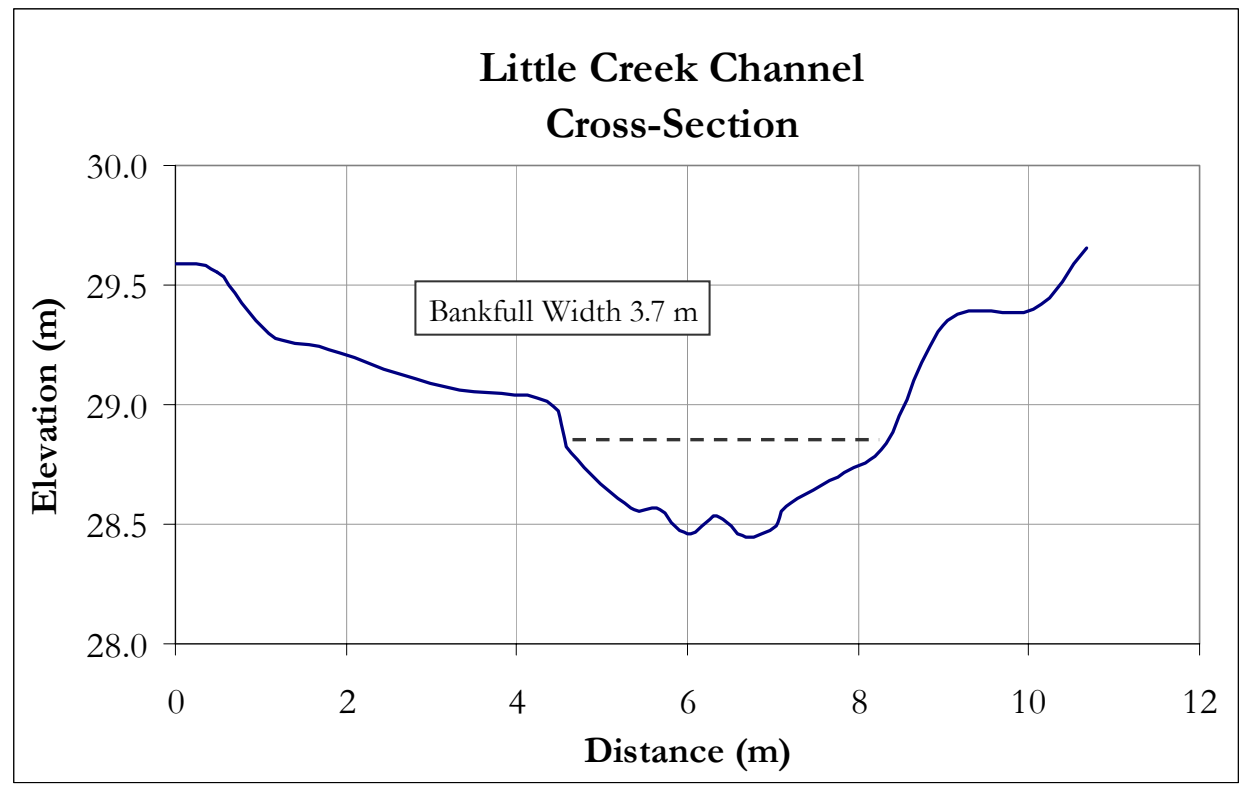

Figure 7. Sample cross section at the Main Stem of Little Creek

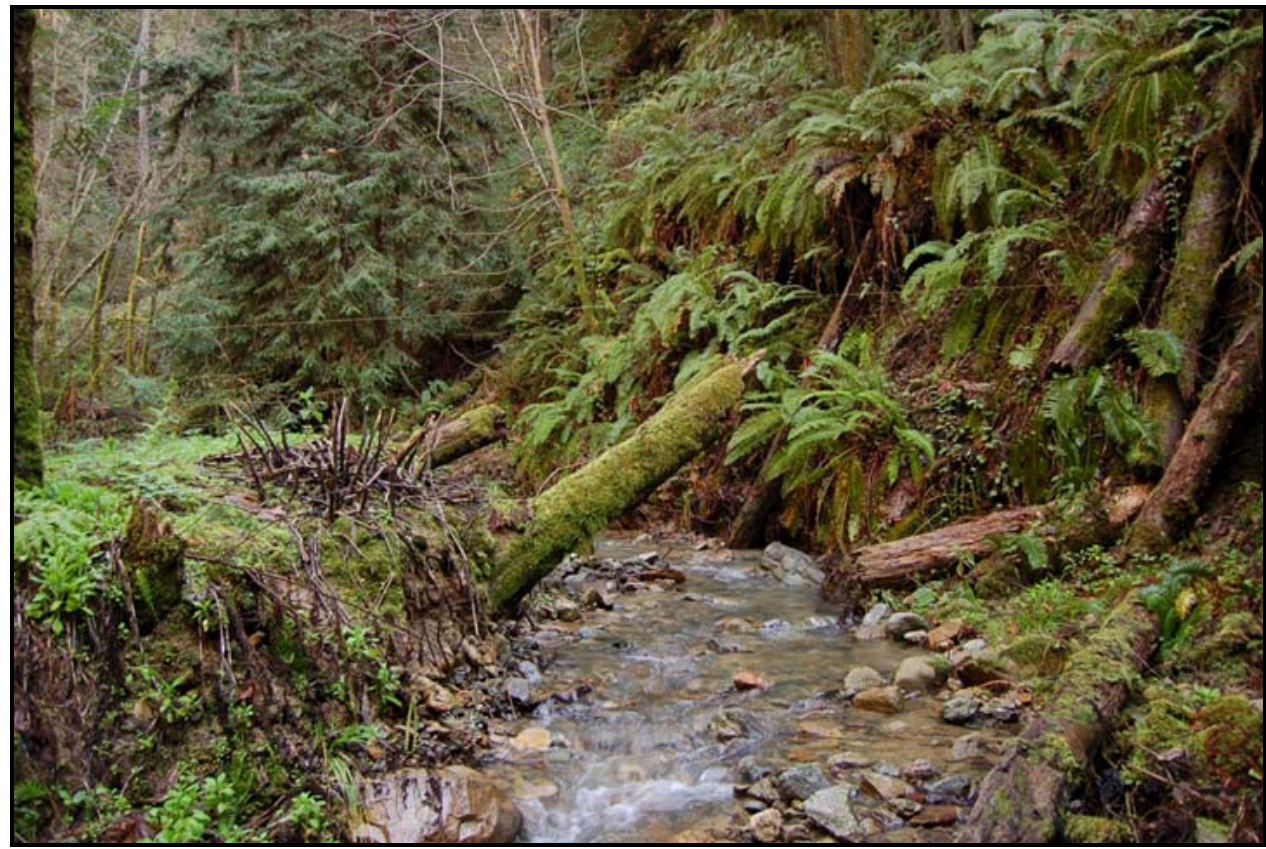

Figure 8. Channel conditions at the Main Stem of Little Creek. 


\section{CHAPTER 4}

\section{Data Collection Methods}

\section{General Approach}

Field-based measurements of the Little Creek road and Little Creek stream channel were compared to measurements obtained using LiDAR terrain data. Field survey control was provided by a licensed land surveying firm to allow for survey data of $3^{\text {rd }}$ order or better. Topographic coordinates and elevations were collected using a Leica total station to define the centerline position of the entire length of the Little Creek road. Similar topographic surveying was conducted to define the thalweg of the Little Creek channel at six pre-defined study reaches. Field survey data was collected in the spring of 2002 and later input into a GIS geodatabase for use with ESRI® ArcMap ${ }^{\mathrm{TM}}$ 9.2.

Airborne LiDAR data was also collected in the spring of 2002. Point data was filtered by the vendor to classify the ground and vegetation returns. The filtered groundreturn points were delivered as shapefiles for use in ArcMap. Further inspection and post-processing of this data was conducted in this study to produce a suitable digital elevation model. Visual interpretation of the shaded relief and slope grids were used to map the position of the target road feature, wile automated terrain analysis was used to map the stream channel location. Shapefiles produced from the ground-surveyed data were considered as the "true" feature position, and shapefiles produced from the LiDAR terrain data were measured relative to the true feature position, in order to assess the accuracy of the LiDAR derived products. 


\section{$\underline{\text { Road Centerline Survey }}$}

A field survey of Little Creek road centerline was completed by the licensed surveying firm, Dunbar and Craig in 2002. The surveyors used high-precision GPS to establish primary control on five nearby High Precision Geodetic Network (HPGN) control points referenced to the North American Datum 1983 with the 1991.35 adjustment. Conventional surveying with a total station was used to collect 222 points defining the road centerline location while an automatic level was used to determine the elevations of 126 centerline points. Based on survey control data, the accuracy of this survey was $0.07 \mathrm{~m}$ Northing, $0.06 \mathrm{~m}$ Easting and $0.07 \mathrm{~m}$ vertical. Coordinate data from the road centerline survey (Figure 9) was transferred from a computer-aided design (CAD) file into point and polyline features in a geodatabase for use in ArcMap.

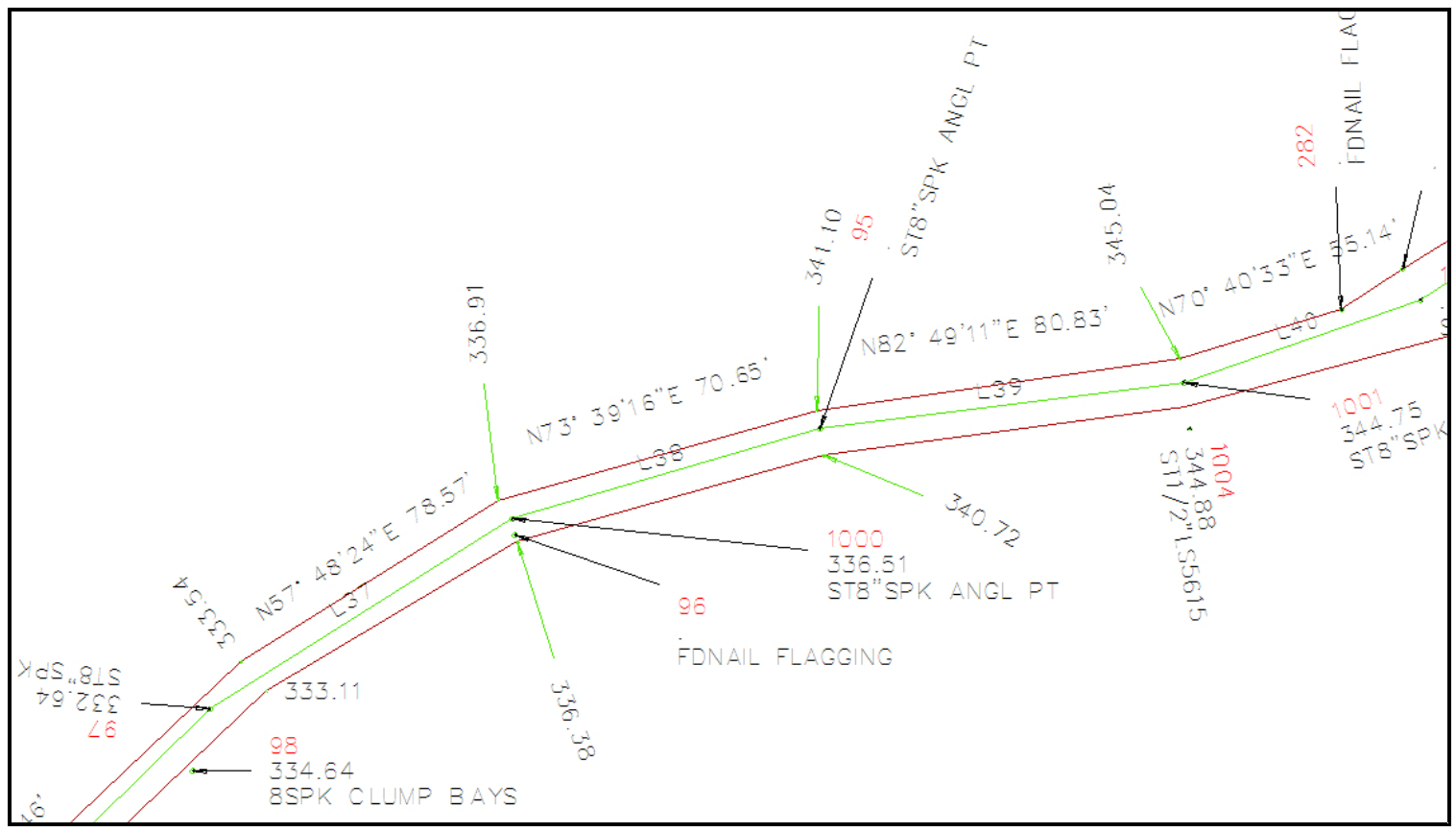

Figure 9. Example of road edges and centerline from ground-based survey represented in a geodatabase. 


\section{Stream Thalweg Survey}

Six study reaches in the Little Creek watershed have been established to monitor long-term changes in channel morphology. These study reaches were specifically located to represent conditions found throughout the watershed (Figure 10). Each reach is approximately $100 \mathrm{~m}$ in length and contains permanent benchmarks for ten cross-section

profile lines spaced $5 \mathrm{~m}$ apart. Annual cross-section surveys at these locations are used to characterize annual and long-term change. The $5 \mathrm{~m}$ spacing of these cross-sections helps to identify morphologic changes that occur at the reach level not just changes observed for a single cross-section.

In addition to annual cross-section measurements, a longitudinal profile is measured along the thalweg of each study reach. Profile data is collected with a total station, which provides horizontal and vertical position of the stream thalweg. Local permanent control points for each study reach have been surveyed into the control network established along the Little Creek road. Field survey data collected in the spring of 2002 was selected from this annual dataset for comparison with the LiDAR data, which was also collected in the spring of 2002. Coordinate and elevation data from the filed survey was converted into a geodatabase for use in ArcMap. 


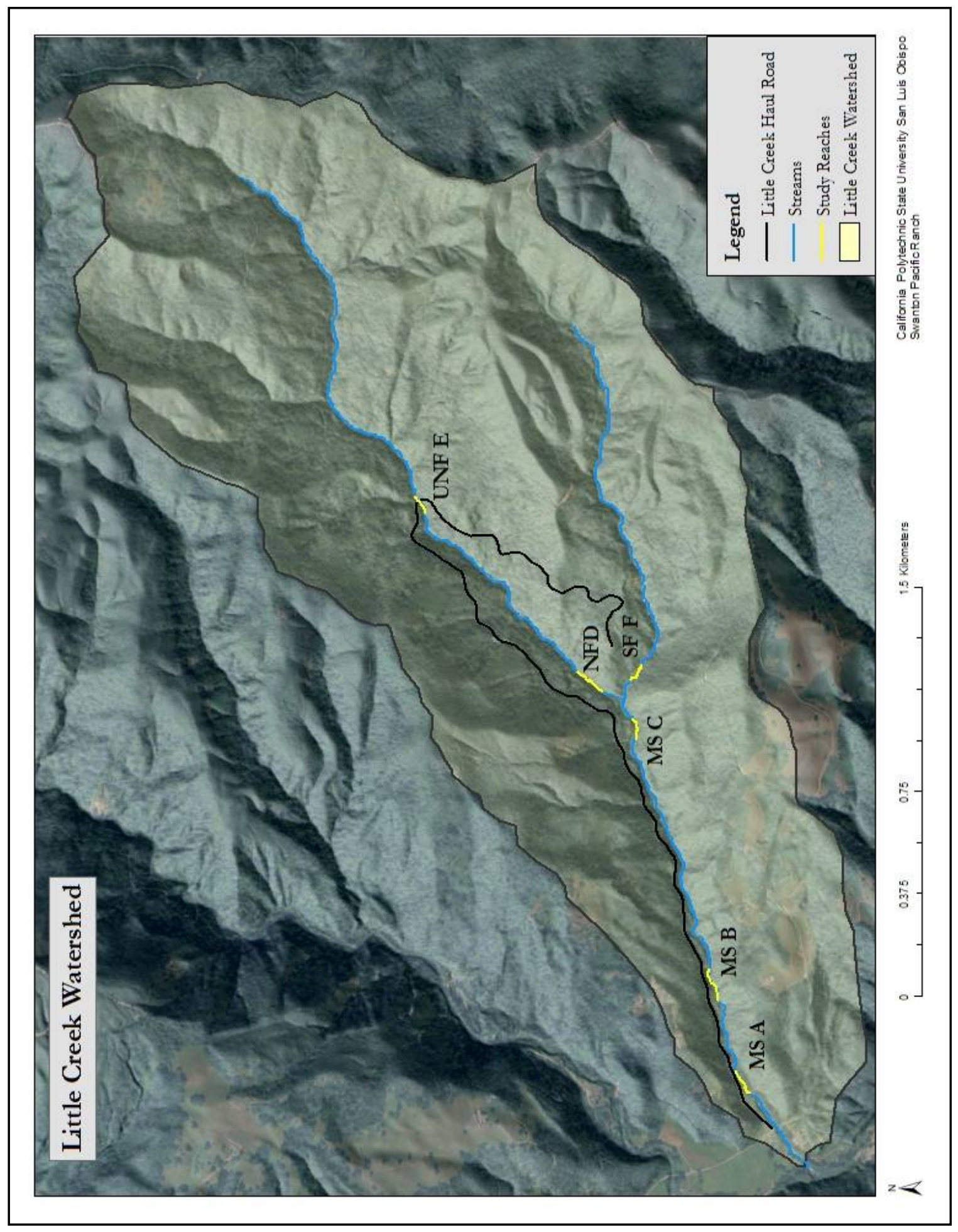

Figure 10. Study reaches in the Little Creek watershed. 


\section{$\underline{\text { LiDAR Data Collection }}$}

Airborne LiDAR was collected for Swanton Pacific Ranch on two separate flight dates by the surveying firm Spencer B. Gross, Inc., Portland, Oregon. The initial flight on March 30, 2002 was only partially completed due to a marine layer of clouds, which created poor survey conditions over the western side of the property. Only eight of thirteen planned flight lines were completed. A second flight occurred on April 28, 2002 where all 13 flight lines were completed. Vegetation conditions at the time of the second flight were considered marginal, as spring foliage for many deciduous species had begun to emerge. The final LiDAR dataset was compiled using suitable data from both flights. Specifications of the LiDAR sensor and flight parameters are summarized in Table 3.

Table 3. LiDAR flight and sensor specifications.

\begin{tabular}{|l|l|}
\hline \multicolumn{2}{|c|}{ LiDAR Survey Parameters } \\
\hline \hline Altitude: & $1,800 \mathrm{~m}$ \\
\hline Flight lines: & 13 \\
\hline Scan angle: & $25^{\circ}$ \\
\hline Swath width: & $567 \mathrm{~m}$ \\
\hline Swath overlap: & $30 \%$ \\
\hline Pulse rate: & 20,000 pulses/sec. \\
\hline Returns: & 3 per pulse (with intensity) \\
\hline
\end{tabular}

The raw data from the flight was post-processed by the vendor to classify points reflected from buildings and vegetation and to isolate the points that reflected from the ground surface. The vendor delivered separate point shapefiles containing classified vegetation returns, classified ground returns, and a $3 \mathrm{~m}$ DEM grid interpolated from the ground return data. 


\section{$\underline{\text { LiDAR Point Data }}$}

The distribution and density of LiDAR returns in the Little Creek watershed were carefully evaluated for the study area. A visual inspection of the LiDAR ground returns (Figure 11) illustrates the typical patterns that were observed. Areas of high ground-point density were achieved in areas with less overstory canopy, while low ground-point density was achieved in areas with dense canopy or ground foliage. In some cases, the outline of individual tree crowns created a distinct gap in the ground point coverage.

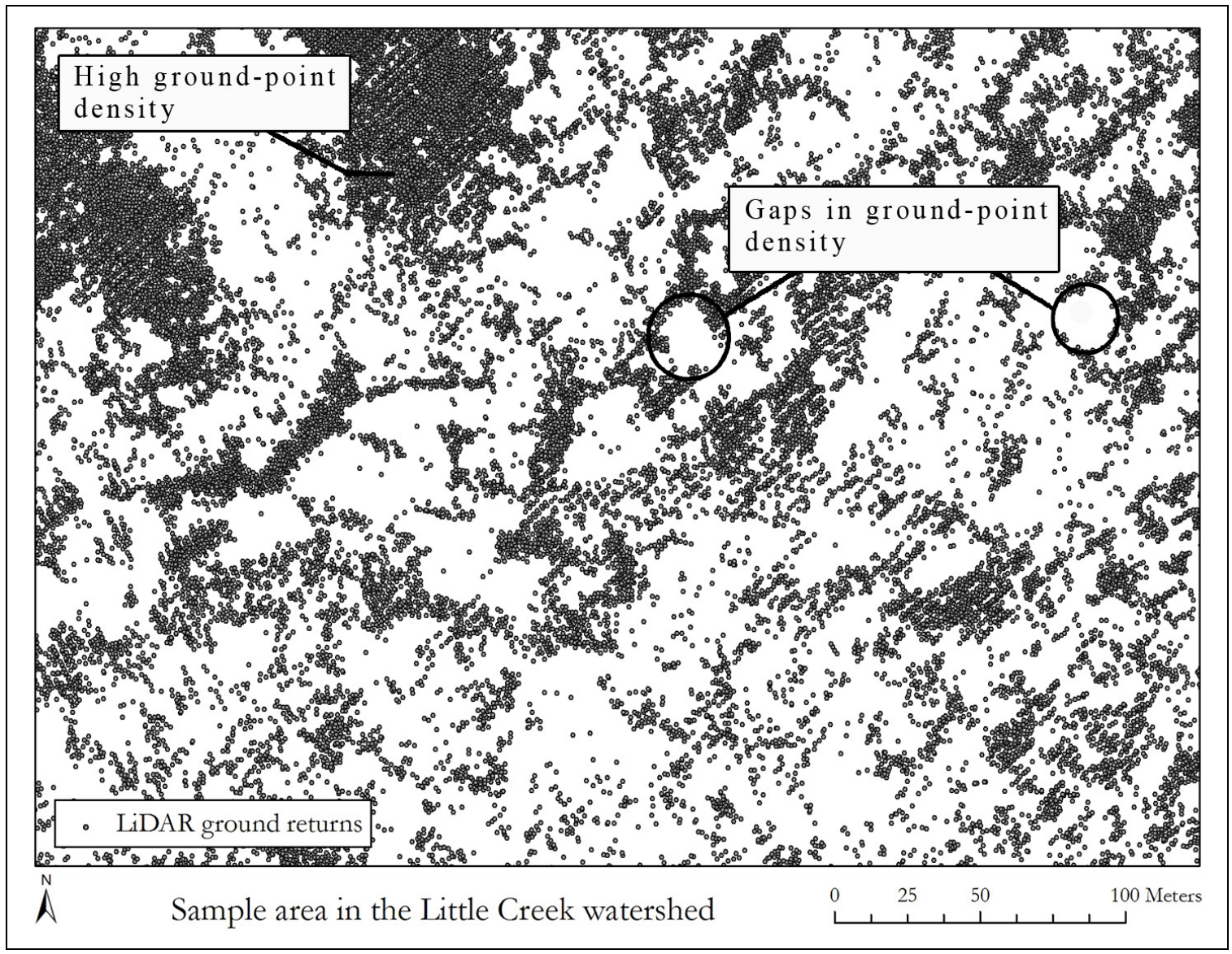

Figure 11. Density and distribution of LiDAR ground returns in a sample area of the Little Creek watershed. 
The summary data in Table 4 shows that $85 \%$ of all LiDAR pulses were classified as canopy or vegetation returns. In particular, the dense and clumped pattern of the coast redwood canopy in the study area reduced the number of pulses that reached the ground, causing gaps and a greater variability in ground point distribution.

Table 4. LiDAR data within the Little Creek watershed.

\begin{tabular}{|l|c|c|}
\hline LiDAR Data Summary & $\begin{array}{c}\text { Average Point } \\
\text { Density }\end{array}$ & $\begin{array}{c}\text { Average Point } \\
\text { Spacing }\end{array}$ \\
\hline \hline All Returns & $1.56 \mathrm{pts} . \mathrm{m}^{2}$ & $0.80 \mathrm{~m}$ \\
\hline Ground Returns & $0.24 \mathrm{pts} . \mathrm{m}^{2}$ & $2.06 \mathrm{~m}$ \\
\hline Percent Vegetation Returns & $85 \%$ & \\
\hline
\end{tabular}

The variation in point spacing for the canopy and ground returns was further quantified by computing the nearest distance from LiDAR returns to 618 sample locations. These sample locations were positioned across the study area in a $100 \mathrm{~m}$ grid with randomized start. From these locations, the average distance to the nearest canopy return was 0.43 meters while the average distance to the nearest ground point was 1.70 meters. Variation in point spacing is presented as a histogram and cumulative frequency in Figure 12. The high density and even spacing of canopy reflected LiDAR returns is illustrated by the histogram in green. The ground returns illustrated in brown, exhibit a much greater average spacing, which is also more variable across the sample locations. 


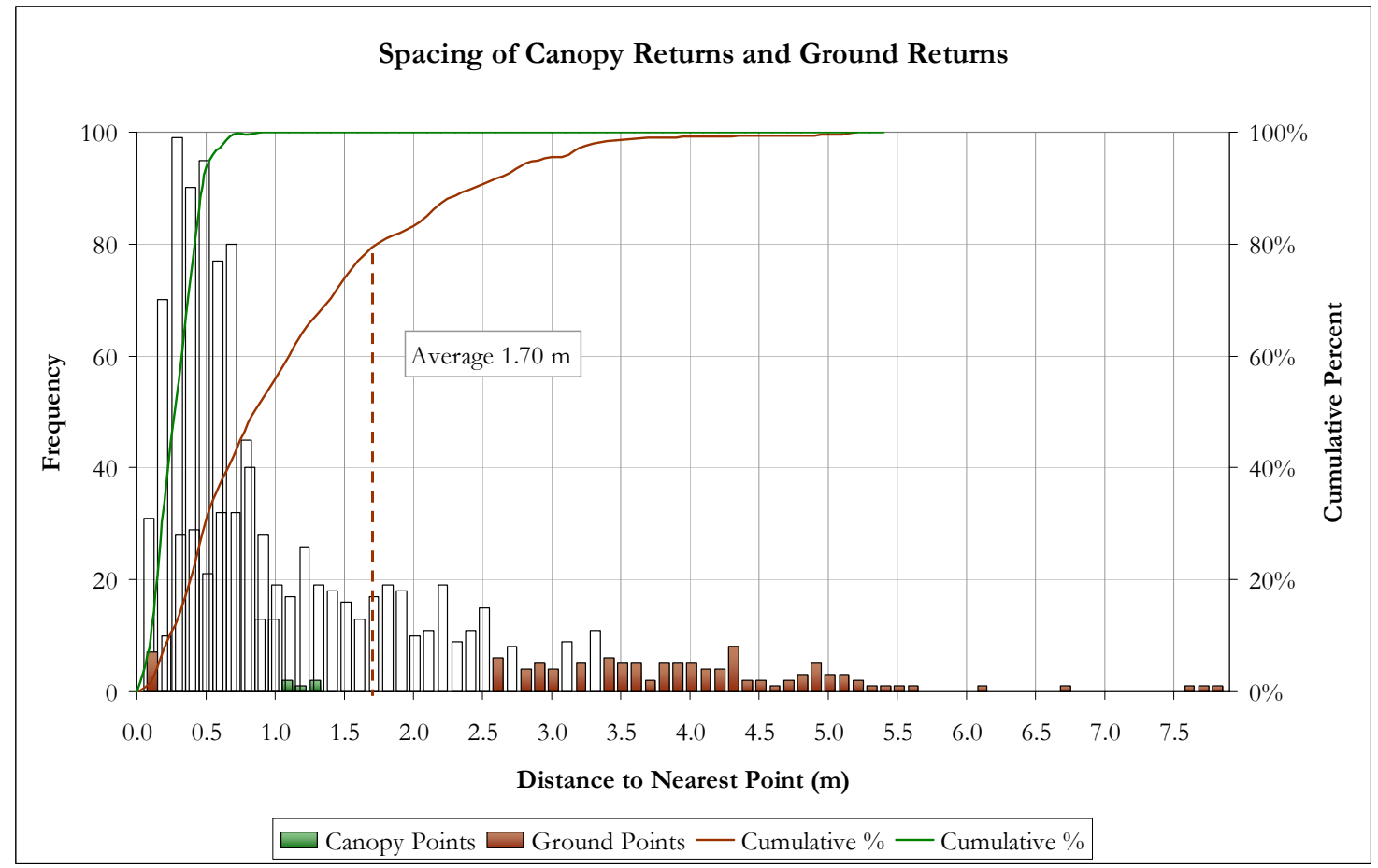

Figure 12. Distribution of LiDAR point spacing.

The density and pattern of LiDAR ground returns is a primary concern since it relates to the resolution or level of topographic detail that the data will provide. Portions of the study area where only sparse ground returns were achieved may reveal less topographic detail than areas of higher point density. The chart in Figure 13 illustrates the fraction of watershed area occupied by five point-distance classifications. For approximately two thirds of the watershed area, the distance to the nearest LiDAR ground point is $2 \mathrm{~m}$ or less. The remaining areas of the watershed received lower point density. Some large gaps were also observed where the distance to the nearest LiDAR ground point was 10 meters or more. 


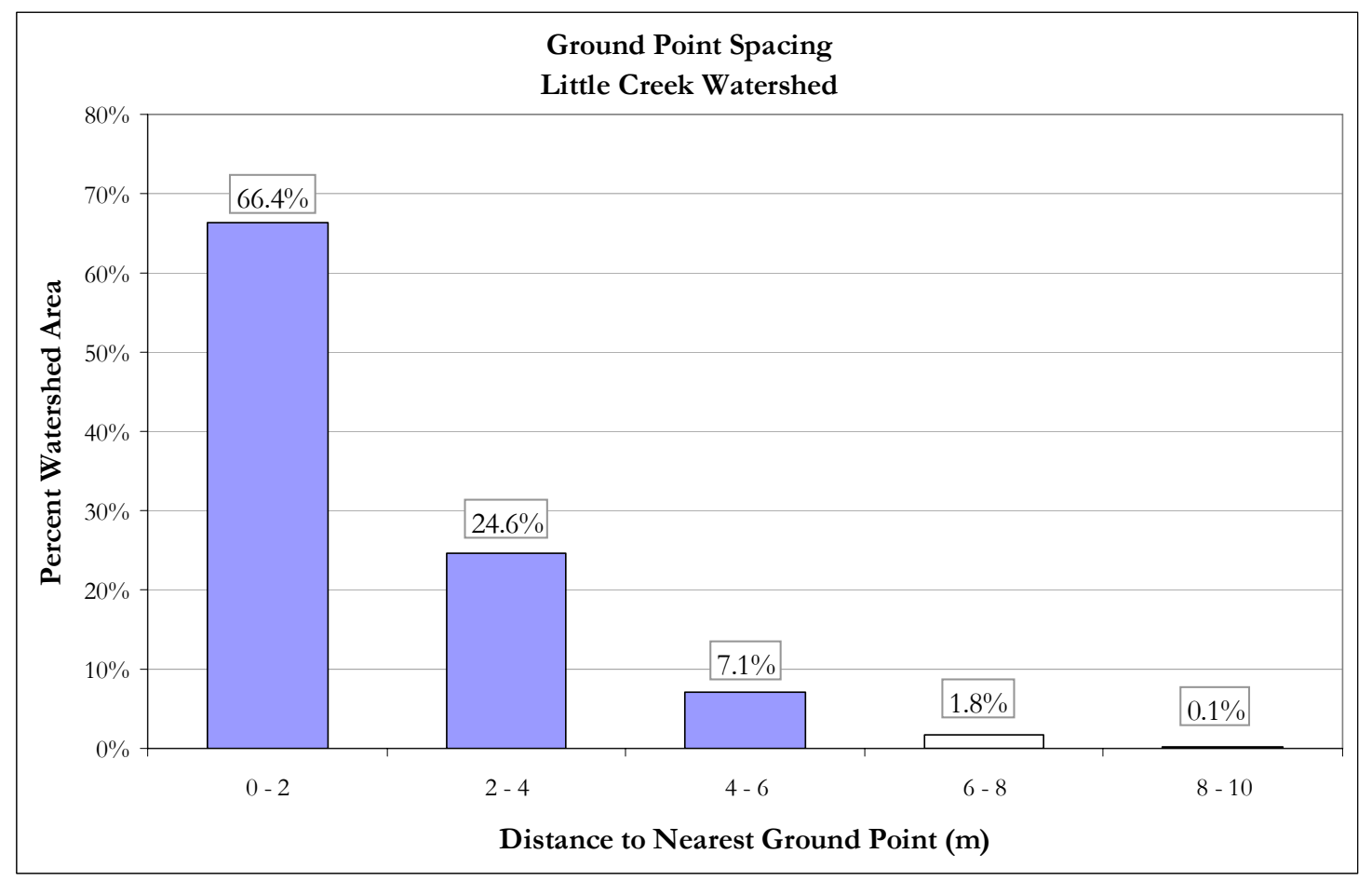

Figure 13. Watershed area classified by ground point spacing.

\section{$\underline{\text { LiDAR-Derived DEM }}$}

The irregular pattern of LiDAR ground returns and associated elevations were gridded into a digital elevation model through the process of interpolation. Producing a high-quality DEM that adequately represents the ground surface is important since the gridded DEM data will be used for both visual interpretation and topographic analysis in this study. The two primary considerations for creating a DEM are selecting an appropriate grid resolution and a suitable interpolation method. Several guidelines for determining a suitable grid resolution are presented in the literature. These guidelines suggest that an appropriate grid size is dependent on the density of input data (Hu, 2003), reflects the complexity of the terrain (Hengl, 2006), though should not contain more data or detail than is necessary for a particular application (Liu, 2008). Generating a highresolution DEM from sparse input data would not be appropriate since the resulting 
surface would be shaped more by the interpolation function rather than the input data. On the other hand, generating a coarse-resolution DEM from a high-density data source, such as LiDAR would not retain the topographic detail of the source data (Liu, 2008).

From the guidelines presented earlier, an appropriate resolution for a DEM generated from the LiDAR ground returns in the study area may range from 0.5 meters to 2 meters. The overall average ground point spacing of 2.06 meters was determined with the equation presented in $\mathrm{Hu}$, (2003). This method determines the simple average point spacing as if the ground points were distributed evenly across the study area.

$$
\bar{S}_{p}=\sqrt{\left(\frac{A}{N}\right)}
$$

Where:

$$
\begin{aligned}
& \bar{S}_{p}=\text { average spacing between ground points (meters) } \\
& \mathrm{N}=\text { total number of ground points } \\
& \mathrm{A}=\text { Area (square meters) }
\end{aligned}
$$

A grid size of $1.5 \mathrm{~m}$ was selected as a suitable DEM resolution to match the overall density of the input data while avoiding an unnecessary computational expense of a higher resolution grid. The interpolation was accomplished in ArcMap 9.2 using the Topo-to-Raster tool, which executes the TopoGrid command from ArcInfo. This interpolation is based on the ANUDEM method which is specifically designed to model terrain surfaces from irregularly spaced elevation point data (Hutchinson, 1989). The advantages of this method are a combination of computational efficiency, high overall surface quality, and improved modeling of drainage characteristics (ESRI, 2006b; 
Hutchinson, 1989). While this method also includes options for the automatic removal of spurious sinks, this option was not selected. The process of sink removal will take place using Arc Hydro, and will be described later.

The choice of DEM grid size is again important since it relates to the size of the smallest topographic features that can be represented and identified in the DEM. A general guideline suggests that a minimum of two pixels is required to identify and represent the width of elongated objects (Hengl, 2006). This guideline is related to the Nyquist frequency concept from the field of signal processing, which states that a signal can be reconstructed if the sampling frequency is two times the original frequency (Shannon, 1949). Given these guidelines, a grid resolution of 1.5 meters should support identification and mapping of road features that are at least 3 meters wide.

\section{Digitized Forest Road Location}

The general LiDAR processing workflow (Figure 14) illustrates the approach used to identify and digitize the Little Creek road feature using the LiDAR data. Classified LiDAR ground returns (Figure 14a) were interpolated into the 1.5 m DEM (Figure 14b). The DEM was used to produce a $1.5 \mathrm{~m}$ shaded relief (Figure 14c) and 1.5 m slope grid (Figure 14d). Finally, visual interpretation of both the shaded relief and slope grids was used to digitize the centerline of the Little Creek road (Figure 14e and 14f). Standardized methods for effective road digitizing were developed. These methods begin by specifying the display settings for the hillshade and slope layers. The shaded relief grid (Figure 14c) was created in ArcMap using the default sun azimuth of $315^{\circ}$ and altitude of $45^{\circ}$ and constructed without modeling shadows. This hillshade layer was 
displayed with a bilinear interpolation to smooth the coarse, pixilated appearance of the grid when viewed at larger map scales.

(a) LiDAR Ground Returns.

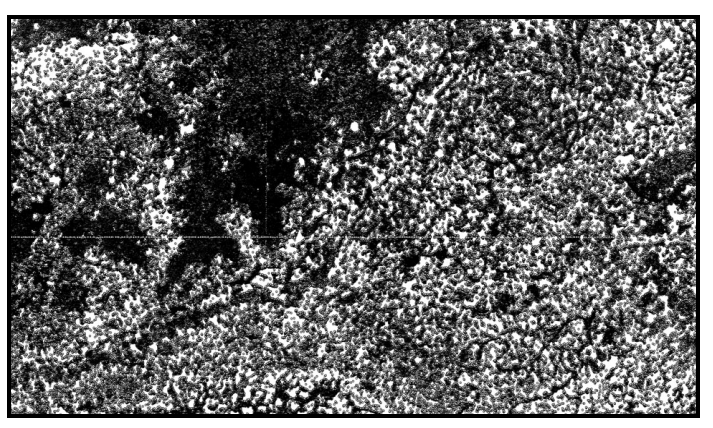

(c) Shaded relief grid.

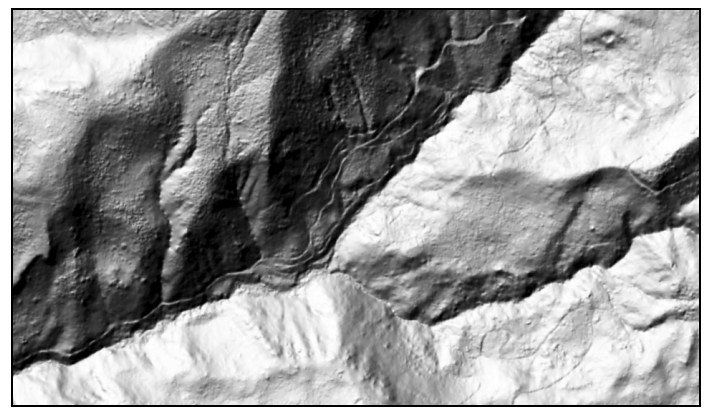

(e) Digitized road feature on shaded relief.

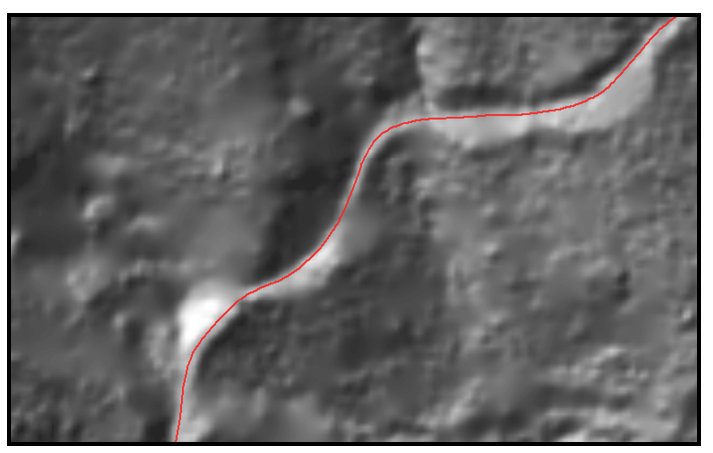

(b) Interpolation to DEM.

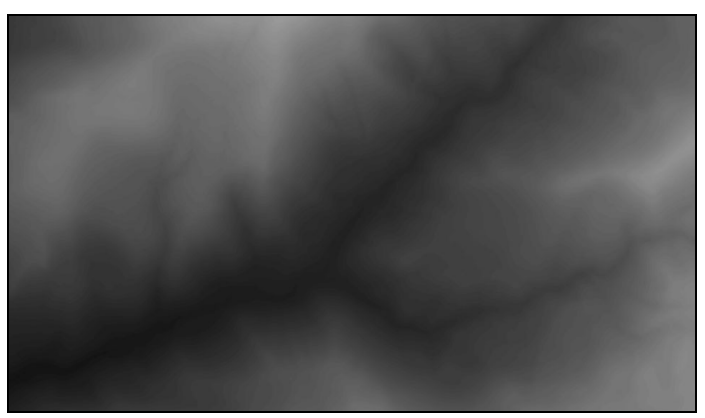

(d) Slope Grid.

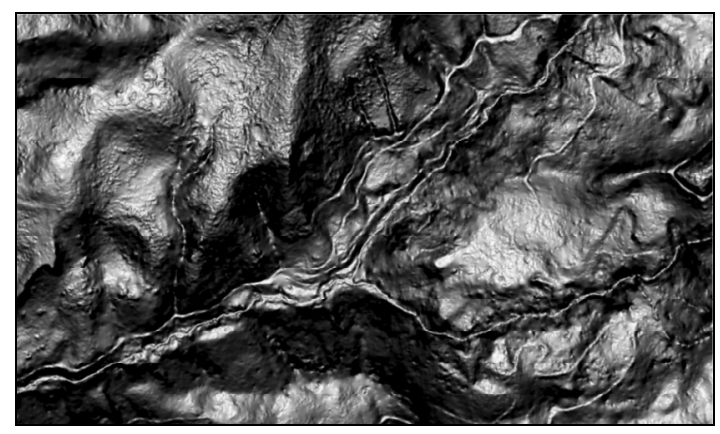

(f) Digitized road feature on slope grid.

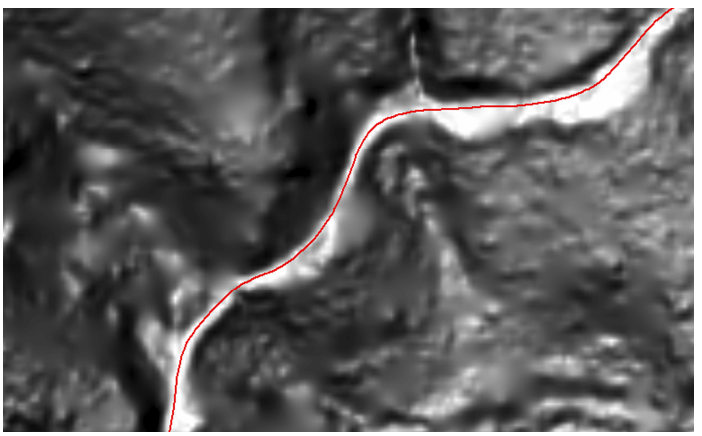

Figure 14. Steps toward digitizing forest road features from LiDAR data 
The contrast of the hillshade was set to adjust to the minimum and maximum values of the current display extent. This setting helps to present the image with a full range of contrast even when panning or zooming across areas of steep vertical relief. The slope grid (Figure 14d) was viewed as stretched grayscale image with low slopes displayed in white and high slope values in black. Displaying the slope layer in this way aided in detecting road features since the low slope of the road surface was highlighted in white, while often paralleled by steeper cut and fill slopes rendered in dark grey or black (Figure 14e) (Rieger et al., 1999).

General assumptions about the geometry of the haul road helped to digitize the

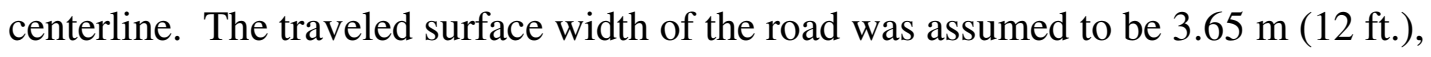
while the width of the entire road bed was expected to be greater. For road segments that appeared wider than $3.65 \mathrm{~m}$, the location of the centerline was digitized to fall within half of the assumed road width, about $1.8 \mathrm{~m}$, from the inside edge of the road, or the cutslope. One useful aid for digitizing was recognizing that the standard digitizing cursor in ArcMap (Figure 15) is two millimeters wide, which equates to two meters in ground units at a map scale of 1: 1,000. By aligning the edge of the cursor to the inside edge of the road at the cutslope it was possible to quickly approximate a $2 \mathrm{~m}$ offset to the road centerline (Figure 15). 


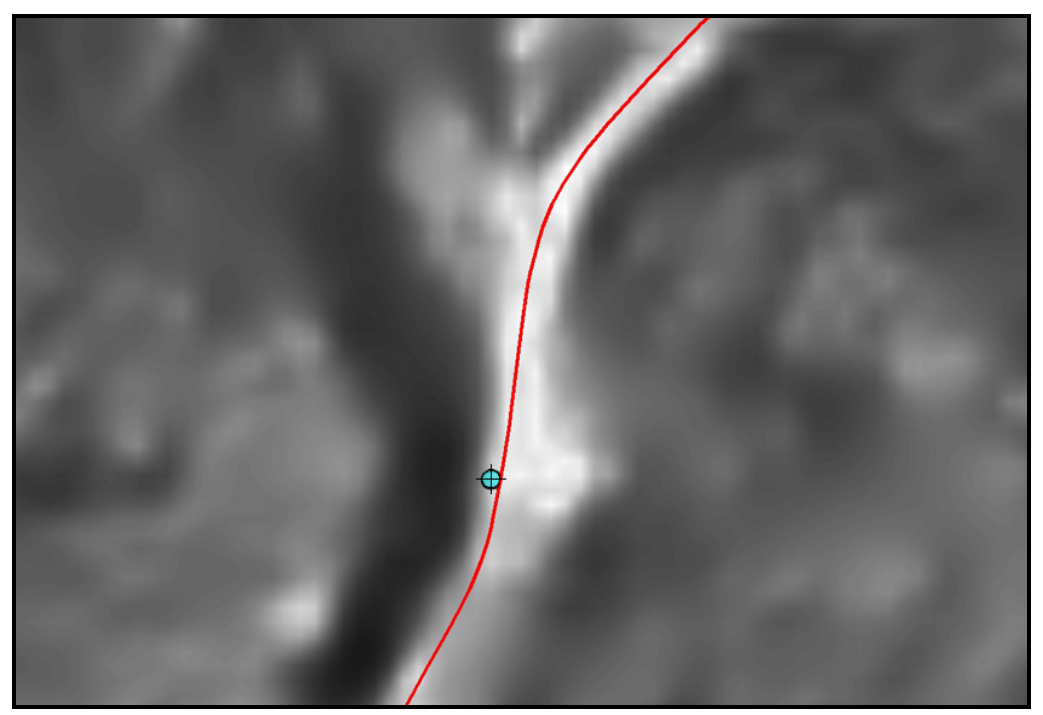

Figure 15. Example of the digitized road centerline and digitizing cursor, with the slope layer in background.

The haul road was digitized at a map scale ranging from 1: 500 to 1: 1,000 and was completed within one hour. Upon completion, the digitized line was smoothed using the polynomial approximation with exponential kernel (PAEK) method in ArcMap's Smooth Line tool. This method uses a moving average technique to smooth the angular appearance of manually digitized lines (ESRI, 2006b). The smoothing parameter was set to use a $15 \mathrm{~m}$ length of road to compute a moving average. This smoothing imparts a minor effect on the horizontal position of the line, though helps the digitized line to match the assumptions of smoothly curving roads (Jenks, 1981). An extensive network of current roads, former railroad grades, and smaller trails were visible within the Little Creek watershed (Figure 16). A total of 27 kilometers (17 miles) of road and trail features were digitized, though only the Little Creek road was used to test the accuracy of the digitized road features. 


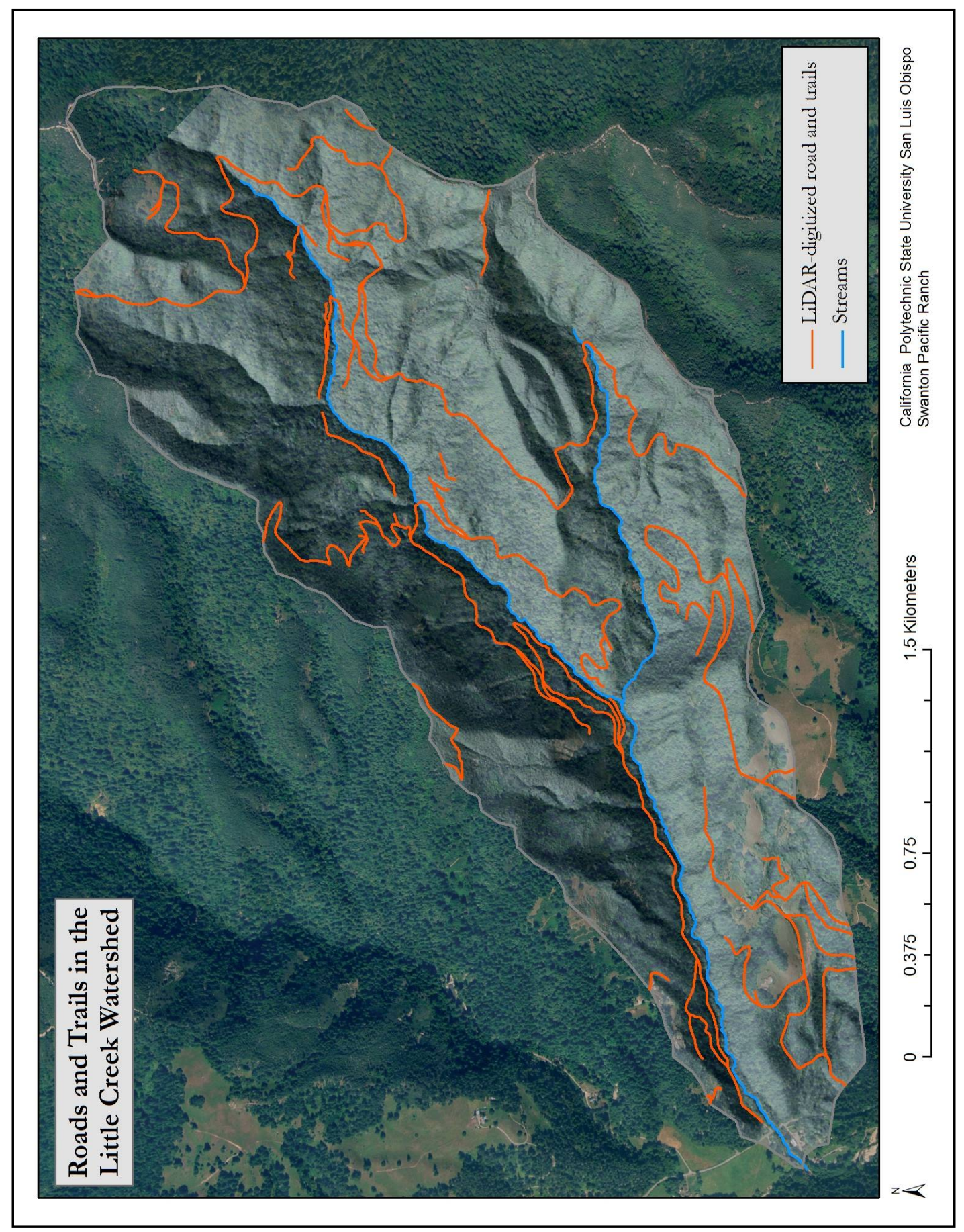

Figure 16. Roads and trail features digitized from LiDAR data within the Little Creek watershed. 


\section{Derived Stream Channel Location}

While the location of the haul road was mapped through a visual interpretation of LiDAR-derived layers, the automated Arc Hydro process (ESRI, 2006a) was used to derive the location of the Little Creek stream channel. Terrain processing with Arc Hydro begins with the option to recondition the DEM to incorporate the location of a preexisting stream channel network. This reconditioning can be used to modify the elevation values of the DEM to ensure that derived channel locations conform to a preexisting layer of stream channel locations (Murphy et al., 2007). This option may be suitable when a highly-accurate stream channel network data source is already available. For the purposes here, the Arc Hydro process is expected to produce a stream network that is more accurate than any existing sources, therefore DEM reconditioning will not be performed.

In the next phase of terrain processing, portions of the DEM are modified in order to fill small spurious sinks, or low points in the DEM, that may have resulted from the original LiDAR data collection or from the interpolation process. Removal of sinks is necessary to ensure that subsequent grid-based calculations of flow direction and flow accumulation will produce a continuous flow path across the landscape. Next, the D8 single flow direction algorithm determined the direction of surface water flow by finding the steepest path from each cell to one of its eight neighbors. Finally, the flow accumulation process determined the number of cells that would flow into or contribute to each cell in the grid, identifying areas of convergent flow (ESRI, 2006a).

At this point, the processing steps that define the stream channel location have generally been completed, though an important step remains. In the Arc Hydro process, a 
drainage area threshold defines the headward initiation point of each channel. If the goal were to define the extent of perennial stream channel flow, then the drainage area threshold would need to approximate the drainage area that coincides with perennial flow for headwater reaches. For the purpose of this study, the drainage area threshold was selected as one percent of the drainage area, the Arc Hydro default setting. The Arc Hydro process results in a raster representation of the stream location. This raster was converted into a polyline using the Spatial Analyst convert raster-to-feature command executed without generalizing the polyline. The final result is a polyline shapefile representing the location of the Little Creek stream channel as derived from the $1.5 \mathrm{~m}$ LiDAR DEM. 


\section{CHAPTER 5}

\section{$\underline{\text { Analysis Methods }}$}

Analyses of LiDAR elevations and LiDAR-derived products were conducted in three steps. First, the LiDAR elevations were compared to field-measured elevation checkpoints. Second, since LiDAR ground returns were interpolated into a DEM, the magnitude of DEM interpolation error was also evaluated. Finally, the LiDAR-derived measurements of the forest road and stream channel features were compared to fieldbased survey measurements.

\section{$\underline{\text { LiDAR Elevation Error }}$}

LiDAR ground-return elevations were compared to 117 field-surveyed checkpoints along the center of the Little Creek road. Only checkpoints that had a LiDAR ground return within a $1.5 \mathrm{~m}$ horizontal distance were included in the analysis.

The single nearest LiDAR ground return for each of these checkpoints was used to assess vertical accuracy. The differences in elevation between the LiDAR and field-surveyed elevations were used to compute the vertical root mean square error for the evaluated LiDAR points.

$$
\mathrm{RMSE}=\sqrt{\frac{\sum_{i=1}^{n} e_{i}^{2}}{n}}
$$

Where:

$$
\begin{aligned}
e_{i}= & \text { difference between survey elevation at point } i \text { and LiDAR elevation at } \\
& \text { point } i . \\
n= & \text { number of elevation checkpoints }
\end{aligned}
$$




\section{$\underline{\text { DEM Interpolation Error }}$}

Vertical error is also introduced through the process of DEM interpolation. A jackknife validation procedure was used to assess the magnitude of these errors, a similar approach is used in other studies (Bater, 2008; Evans and Hudak, 2007; Liu et al., 2007). Ground returns within the Little Creek watershed were partitioned into two groups. A $3 \%$ sample totaling 36,947 randomly selected points was designated as a verification dataset, while the remaining $97 \%$ of the ground-returns were designated as the test dataset. A $1.5 \mathrm{~m}$ resolution DEM was generated from the test dataset using four different interpolation methods; inverse distance weighted, natural neighbor, TopoGrid and the triangular irregular network (TIN to Raster) with linear interpolation. One additional $3 \mathrm{~m}$ DEM was generated using the TopoGrid method to determine the interpolation errors of a lower grid resolution. Once these DEMs were created, the 3\% verification dataset, which was withheld from the interpolation process, was overlaid upon each of the DEMs. The vertical difference between the verification dataset points and the three test DEM surfaces was computed. These vertical differences measure the ability of the three DEM interpolation methods to predict or match the elevation values when LiDAR ground returns are not present.

\section{$\underline{\text { Feature Positional Error }}$}

The positional accuracy of the digitized road and stream channel were compared to the field-surveyed data sources. A simple method for measuring the positional accuracy of digitized linear features is presented by Goodchild and Hunter, (1997). This approach compares a linear feature of high accuracy to a feature of lower accuracy, and 
determines the percentage of the low accuracy line that falls within a specified horizontal distance normal to the high accuracy line. In the case of the Little Creek road, the method was used to answer the following question. What percentage $(y)$ of the digitized haul road falls within $x$ meters normal to the surveyed road centerline? An example of this method is shown in Figure 17 with the surveyed centerline in black, the LiDAR digitized road line in red, and the buffer containing 95\% of the LiDAR-digitized road shown in light green. A small segment of the digitized line that falls outside of the buffer is blue.

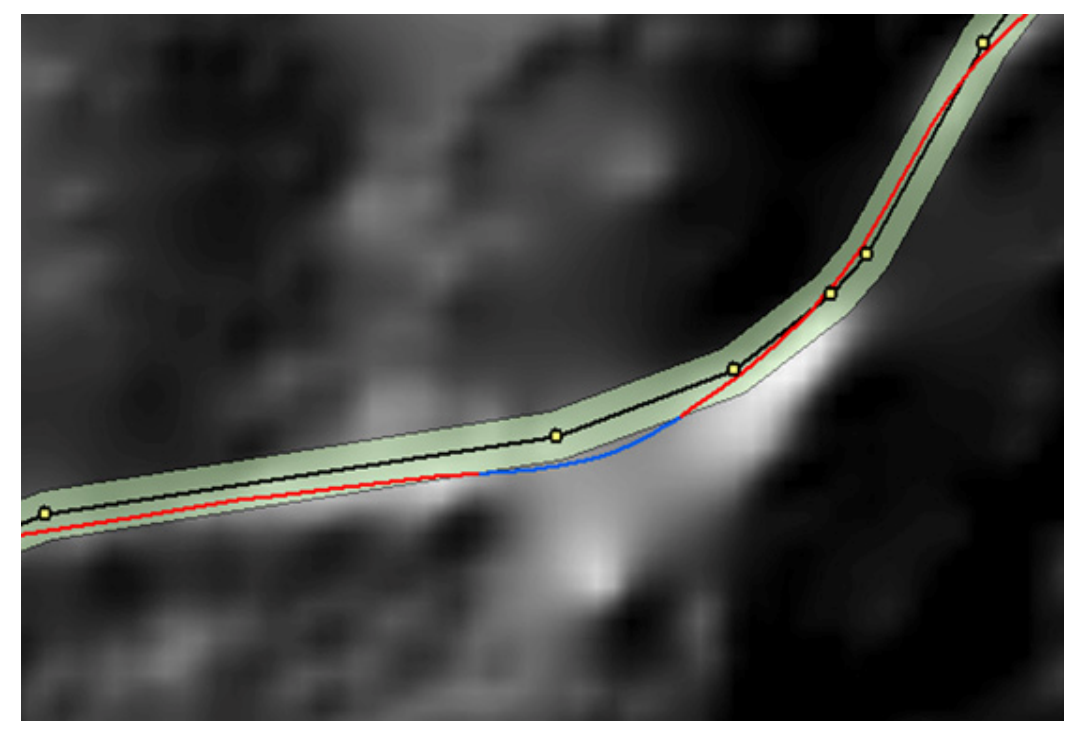

Figure 17. Little Creek road segment with buffer analysis.

The following method is presented by Goodchild and Hunter, (1997) for determining the distance $x$ such that $\mathrm{p}(x)=y$

Where:

$\mathrm{p}=\mathrm{a}$ function which outputs the proportion of a test line (red) lying within a buffer x (green).

$\mathrm{x}=\mathrm{a}$ two-sided buffer distance (green) around the reference centerline (in black)

$\mathrm{y}=$ the desired proportion of test line to be enclosed by buffer $\mathrm{x}$ 
1. Set $\mathrm{x}_{0}=0$ and $\mathrm{p}_{0}=0$ and select a target percentile $y$. Select an initial value $x_{i}$ based on an initial estimate of positional accuracy. Set $\mathrm{i}=1$

2. Construct a buffer of width $x_{i}$ around the reference feature; intersect this buffer with the test feature, and compute the proportion $p_{i}$ of the test feature which falls within the buffer $x_{i}$. If $p_{i}-y<0.001$, then stop.

3. Compute a new estimate of $x$ based on the linear approximation to the function $p$ :

$$
x_{i+1}=\frac{\left(y-p_{i-1}\right)\left(x_{i}-x_{i-1}\right)}{\left(p_{i}-p_{i-1}\right)}+x_{i-1}
$$

4. Set $\mathrm{i}=\mathrm{i}+1$ and go to step 2 .

This iterative search will build an increasingly wide buffer around the reference feature until a sufficient proportion of the test line length is enclosed. A Python script was written to automate this procedure for use in ArcMap (Appendix A). This analysis method was applied to both the road and stream centerlines and determined the buffer width that enclosed $95 \%$ of the LiDAR-derived feature length.

\section{Feature Slope Error}

Longitudinal profiles for LiDAR-derived road and channel features were compared to field-surveyed profiles to evaluate the accuracy of slope measurements. During the field survey of the Little Creek road, survey elevations were collected at significant breaks in road slope. The change in elevation between survey points divided by the horizontal distance between points was used to compute a series of 116 road slope measurements. The average distance between ground survey points was slightly greater than 30 meters. Road slope varied from zero to twenty percent with an average slope of 
eight percent. For the LiDAR-derived profile, the elevation of LiDAR ground returns that fell within a $1.5 \mathrm{~m}$ radius of the ground-surveyed breaks in slope were used to compute a matching series of road slope measurements. A paired t-test was used to determine if there was a statistically-significant difference between the surveyed road slope and LiDAR-derived road slope.

The slopes of LiDAR-derived channel profiles were compared to those of fieldmeasured longitudinal profiles for each of the six study reaches. LiDAR-derived profiles were generated by selecting the LiDAR ground returns that fell within a $0.3 \mathrm{~m}$ distance normal to the Arc Hydro-defined channel position. Horizontal distance from the start of the reach was measured, as if these points fell exactly on the profile line. The slope for these profiles was determined with a simple linear regression line. Regression lines provided slopes for both the field-measured and LiDAR-derived profile for each of the six study reaches. The differences in slope obtained by the two methods were evaluated and summarized though no statistical test was performed due to the low sample size of only six reach comparisons. 


\section{CHAPTER 6}

Data Analysis and Results

\section{$\underline{\text { LiDAR Elevation Error }}$}

The vertical accuracy of 117 LiDAR elevation points was tested through a comparison to 117 ground-surveyed checkpoints along the Little Creek road. Differences in elevation ranged from $-0.74 \mathrm{~m}$ to $0.35 \mathrm{~m}(-2.24 \mathrm{ft}$. to $1.15 \mathrm{ft}$.) The average difference between LiDAR and survey elevations was $-0.19 \mathrm{~m}(-0.6 \mathrm{ft}$.) indicating a potential bias in the LiDAR data. The negative value indicates that the LiDAR elevations were lower on average than the surveyed ground elevation. The RMSE for points along the Little Creek road was $0.29 \mathrm{~m}(0.94 \mathrm{ft}$.$) and falls within the range of vertical accuracies reported for$ forested areas. Reporting the RMSE provides an indication of the unsigned error and variance. If the measured errors are normally distributed and centered on zero, then the RMSE can be considered similar to the standard deviation. Here, the $-0.19 \mathrm{~m}$ bias in LiDAR elevation is incorporated into the RMSE and inflates the apparent variance. A plot of LiDAR elevation errors (Figure 18) illustrates the range in elevation errors with a slight negative bias. One outlier with an elevation error of $-1.49 \mathrm{~m}$ was over five standard deviations away than the mean error. The horizontal position of this point suggested that it may have been a LiDAR return representing the steep downhill fill slope, not the elevation of the road bed so this point was removed from the analysis. 


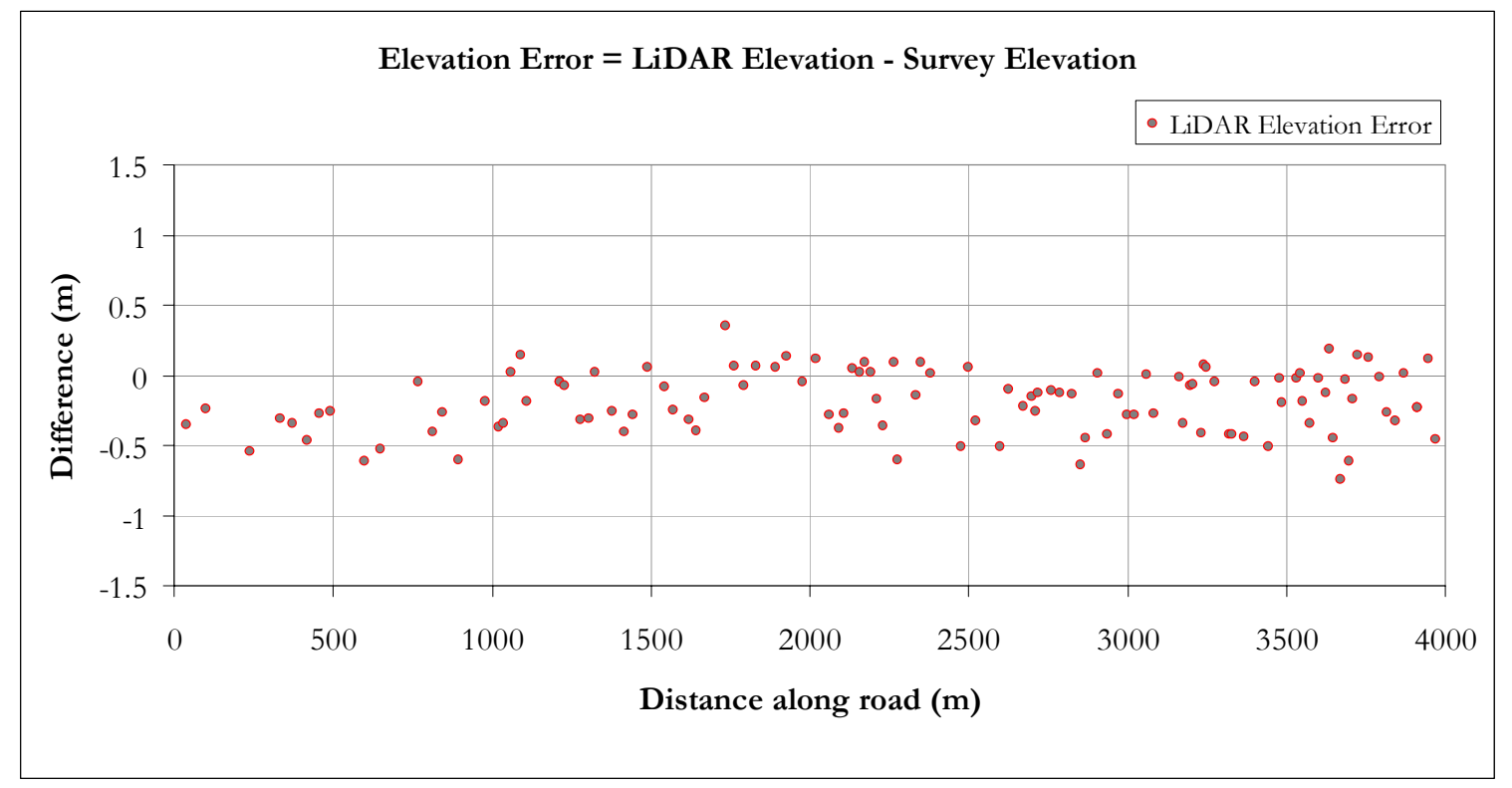

Figure 18. LiDAR elevation errors.

Point elevations from the DEM were also compared to ground-survey elevations. Mean difference in elevation at the same 117 checkpoints was $-0.09 \mathrm{~m}$ while the RMSE was $0.27 \mathrm{~m}$. As seen in Figure 19, these errors may have a slight negative bias, but also contain a greater number of larger positive errors.

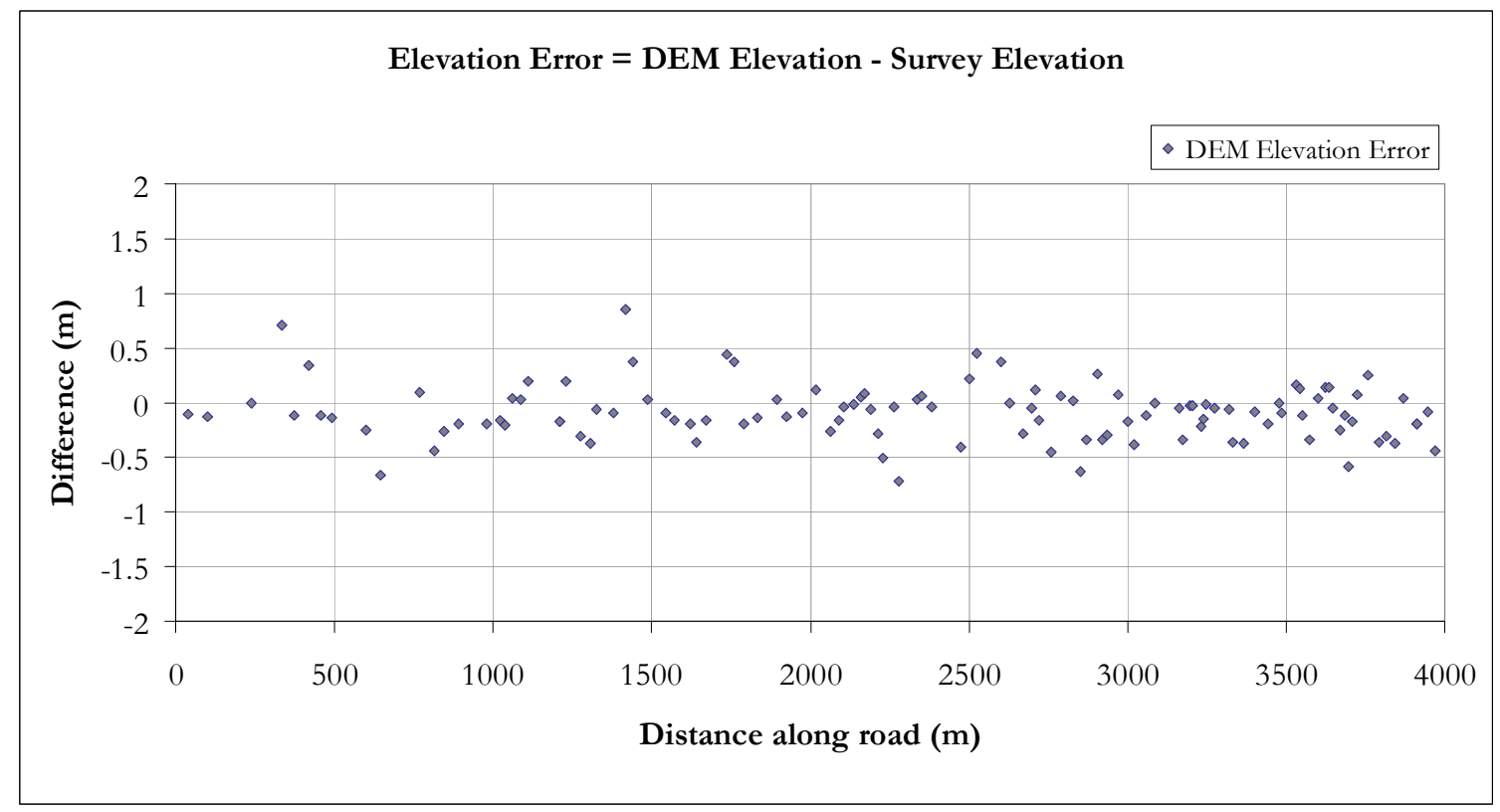

Figure 19. DEM elevation errors. 


\section{$\underline{\text { DEM Interpolation Error }}$}

Elevation errors introduced through the process of DEM interpolation were evaluated for $1.5 \mathrm{~m}$ resolution DEMs produced using four different interpolation methods. A summary of DEM interpolation errors from the TIN, natural neighbor, TopoGrid, and inverse distance weighted (IDW) methods are presented in Table 5. The elevation error from $3 \mathrm{~m}$ resolution DEM produced by the TopoGrid method was also evaluated.

Table 5. DEM interpolation errors.

\begin{tabular}{|l|c|}
\hline Interpolation Method & RMSE (m) \\
\hline \hline TIN to Raster (linear) & 0.46 \\
\hline Natural Neighbor & 0.46 \\
\hline TopoGrid $1.5 \mathrm{~m}$ & 0.50 \\
\hline IDW & 0.56 \\
\hline TopoGrid $3 \mathrm{~m}$ & 0.76 \\
\hline
\end{tabular}

These results indicate a range of interpolation errors introduced among different methods. Constructing a TIN from the original LiDAR ground returns and then converting the TIN to a $1.5 \mathrm{~m}$ resolution grid produced the smallest interpolation errors. Similar results were obtained with the natural neighbor interpolation, which also had a smoother and more appealing visual quality than the TIN method. The TopoGrid method at $1.5 \mathrm{~m}$ resolution resulted in a slightly larger RMSE, while the error introduced by inverse distance weighted method was slightly grater. A larger error was observed for interpolation of the $3 \mathrm{~m}$ DEM. The histogram of interpolation errors for the $1.5 \mathrm{~m}$ TopoGRID DEM (Figure 20), displays a symmetric, nearly unbiased distribution of errors. Given the standard deviation of 0.5 meters, approximately $95 \%$ of the interpolation error is contained within the range of -1.0 to 1.0 meters. 


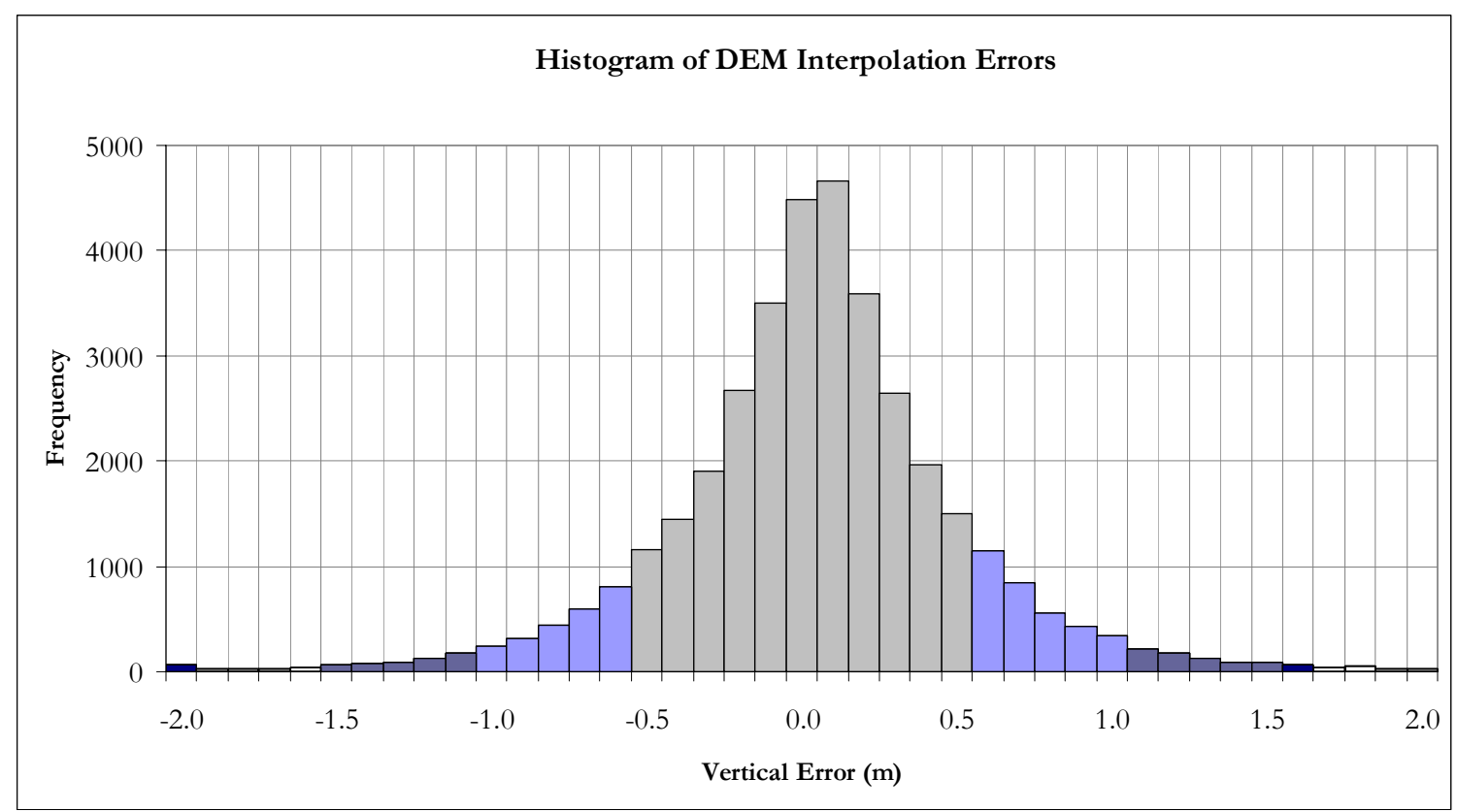

Figure 20. Histogram of DEM interpolation errors. The range of vertical error encountered within one standard deviation of the mean are shaded in grey, while errors occurring at two, three and four standard deviations from the mean are indicated in darker shades of blue.

The map in Figure 21 depicts the spatial pattern of interpolation errors from the TopoGrid method as assessed through the jackknife validation. Contiguous areas of higher interpolation error (areas in red) may result from having a low LiDAR ground point density over areas of complex terrain or sharp breaks in slope. It is important to note that the interpolation error was verified with respect to the LiDAR elevation points, not the true ground elevation. Both the DEM and LiDAR points have been measured to have nearly $0.3 \mathrm{~m}$ vertical error relative to the true ground surface as presented in the previous analysis. 


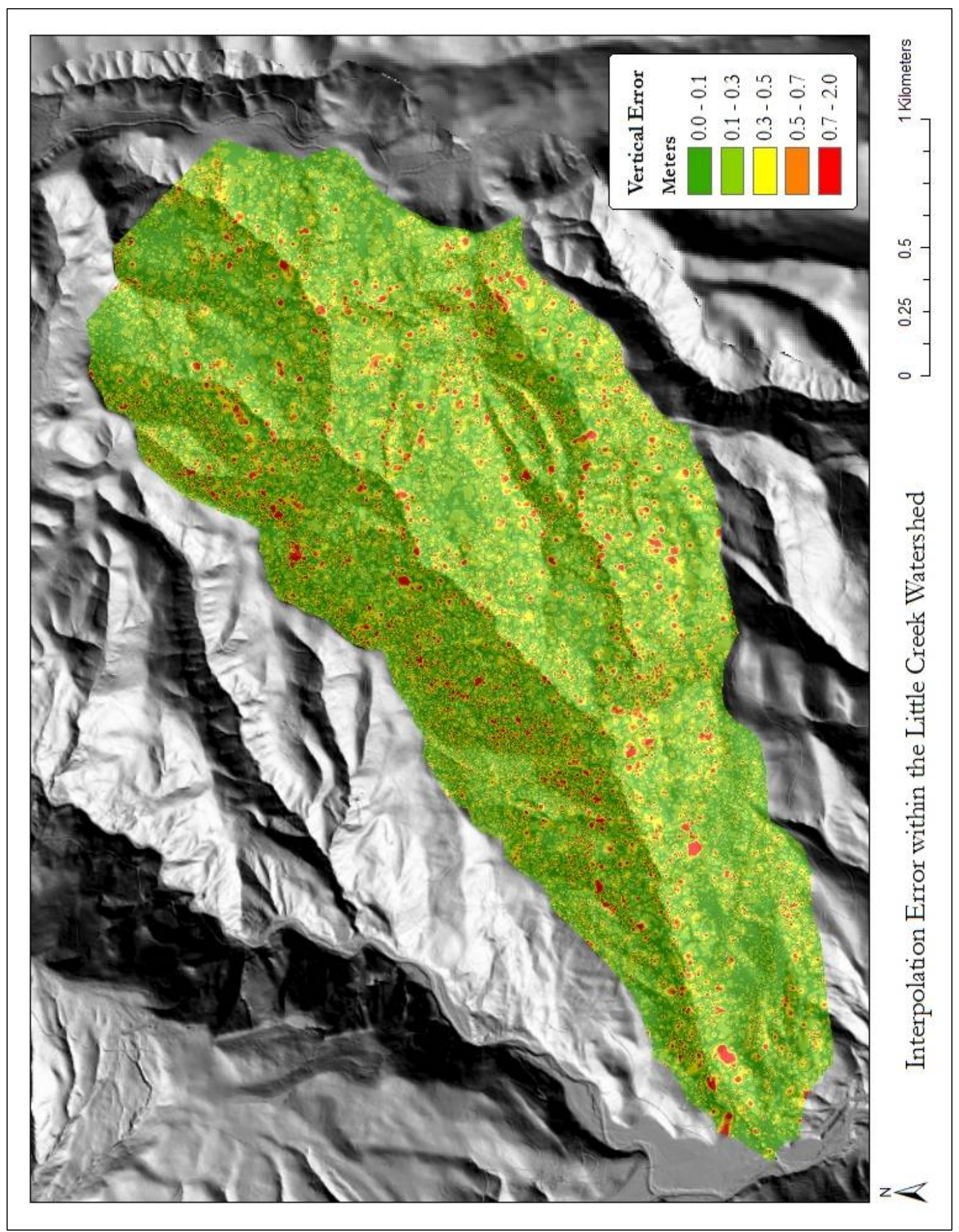

Figure 21. Interpolation errors within the Little Creek watershed. 
A hillshade layer from the DEMs produced by each of the interpolation method were visually examined to determine their suitability for digitizing the Little Creek road feature. The DEM and hillshade produced by the TopoGrid interpolation method was selected due to the combination of a relatively low RMSE and for the visual quality of the hillshade that was produced.

\section{$\underline{\text { Evaluating LiDAR-Derived Features }}$}

\section{Completeness}

Using the $1.5 \mathrm{~m}$ LiDAR-derived DEM a total of $27.2 \mathrm{~km}$ of roads, skidtrails, and other recreational trails were identified and mapped throughout the Little Creek watershed. For comparison, only $4.9 \mathrm{~km}$ or $18 \%$ of that total road length could be identified using a $1 \mathrm{~m}$ color orthophoto. Even for the prominent Little Creek road, only $18 \%$ of its length could be directly mapped with the orthophoto. In contrast, $100 \%$ of the Little Creek road length was mapped through visual interpretation of both the LiDAR hillshade and slope layers.

While most of the Little Creek road was easily identified from the LiDAR-derived layers, two types of problems were encountered. First, portions of the study area that received few or no LiDAR ground returns resulted in areas of poorly defined topography. Gaps in LiDAR point coverage along the Little Creek road caused gaps in the DEM surface detail, and the road was not clearly defined in those areas (Figure 22). 


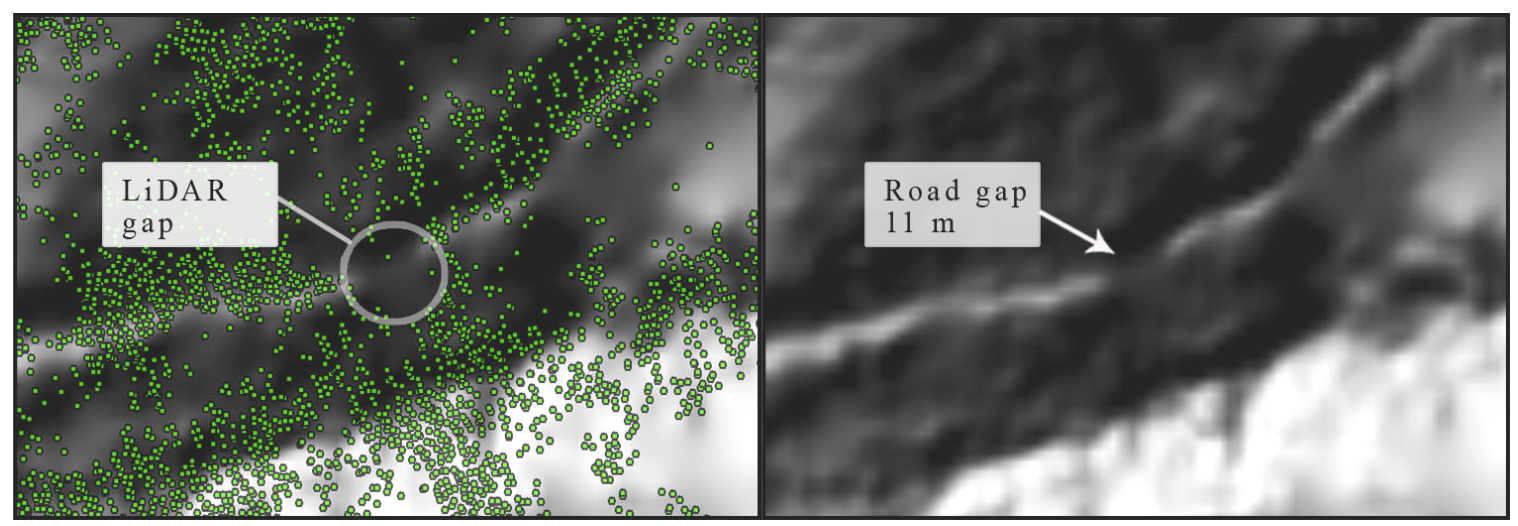

Figure 22. A gap in LiDAR ground returns (left) resulted in a gap in road topography (right).

Gaps in LiDAR ground point coverage resulted in 63 gaps in road topogaphy, amounting to $400 \mathrm{~m}$ in length, about $10 \%$ of the total haul road length. These gaps were relatively short, averaging 14 meters and with a maximum length of 21 meters. While these gaps did not display the expected road topography, it was still possible to approximate the location of the road centerline. Generally, these gaps were not large enough to cause confusion over the correct road location.

The second problem encountered when digitizing the haul road occurred where the road traversed areas with low topographic relief. In these areas, the haul road is not defined by the steep road cuts of fill slopes, as with roads built on steeper hillslopes (Figure 23). These segments accounted for an additional $100 \mathrm{~m}$ of poorly-defined road length. These areas received sufficient LiDAR ground point coverage, but it was the lack of vertical relief in the road feature that caused these road segment to be more difficult to identify. 


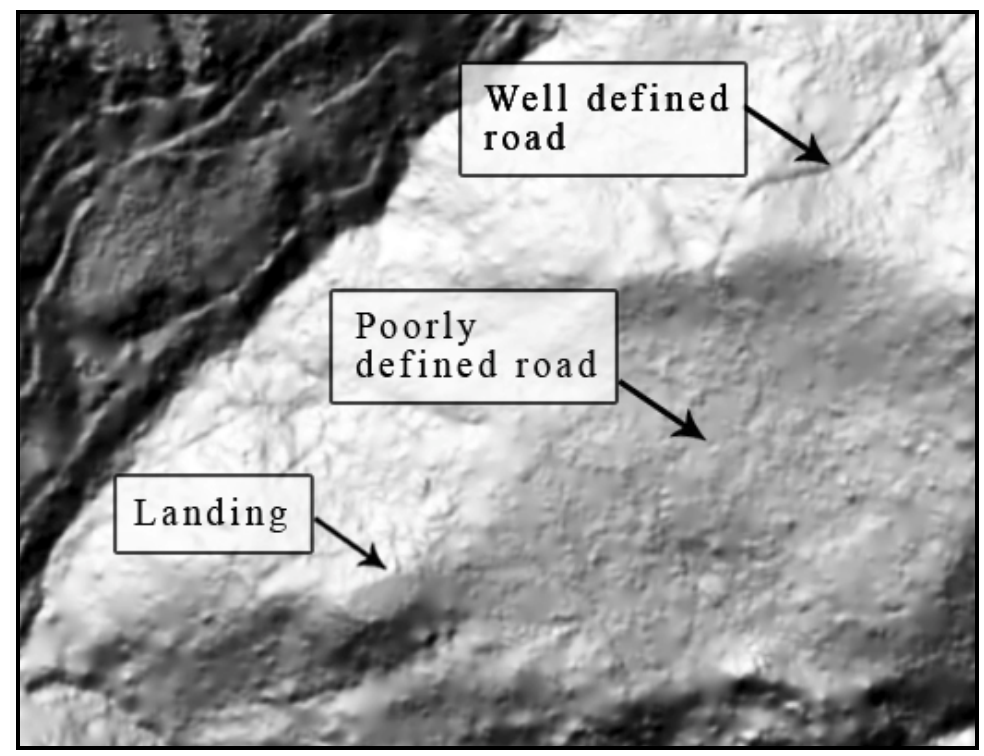

Figure 23. Examples of well defined and poorly defined road segments.

While it was more difficult to recognize road characteristics on segments of flat topography, it was still possible to identify the correct road location. Viewing the original LiDAR ground returns for this area provided an additional visual cue for identifying the road. The lack of vegetation along the road typically results in a high density of LiDAR ground returns along the road bed (Figure 24) (Lee et al., 2005).

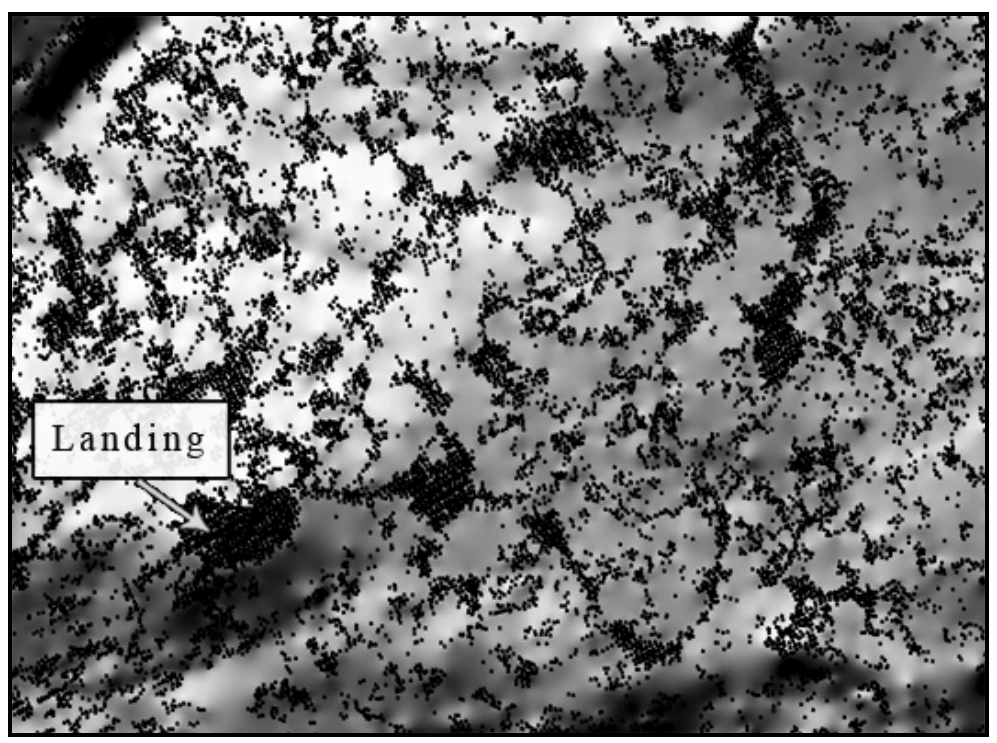

Figure 24. High density of LiDAR points along the road. 
Roads in areas of low relief demonstrated much more subtle topographic characteristics compared to roads in steeper terrain. To illustrate this further, two road cross-sections were generated for sample locations on well-defined and poorly-defined road segments. Figure 25 illustrates the distinct topographic characteristics of the road cross section including the road bench and cutslope. Vertical relief from the road surface to the top of the cutslope is about $20 \mathrm{~m}$ while the overall width of the feature is about 10 meters.

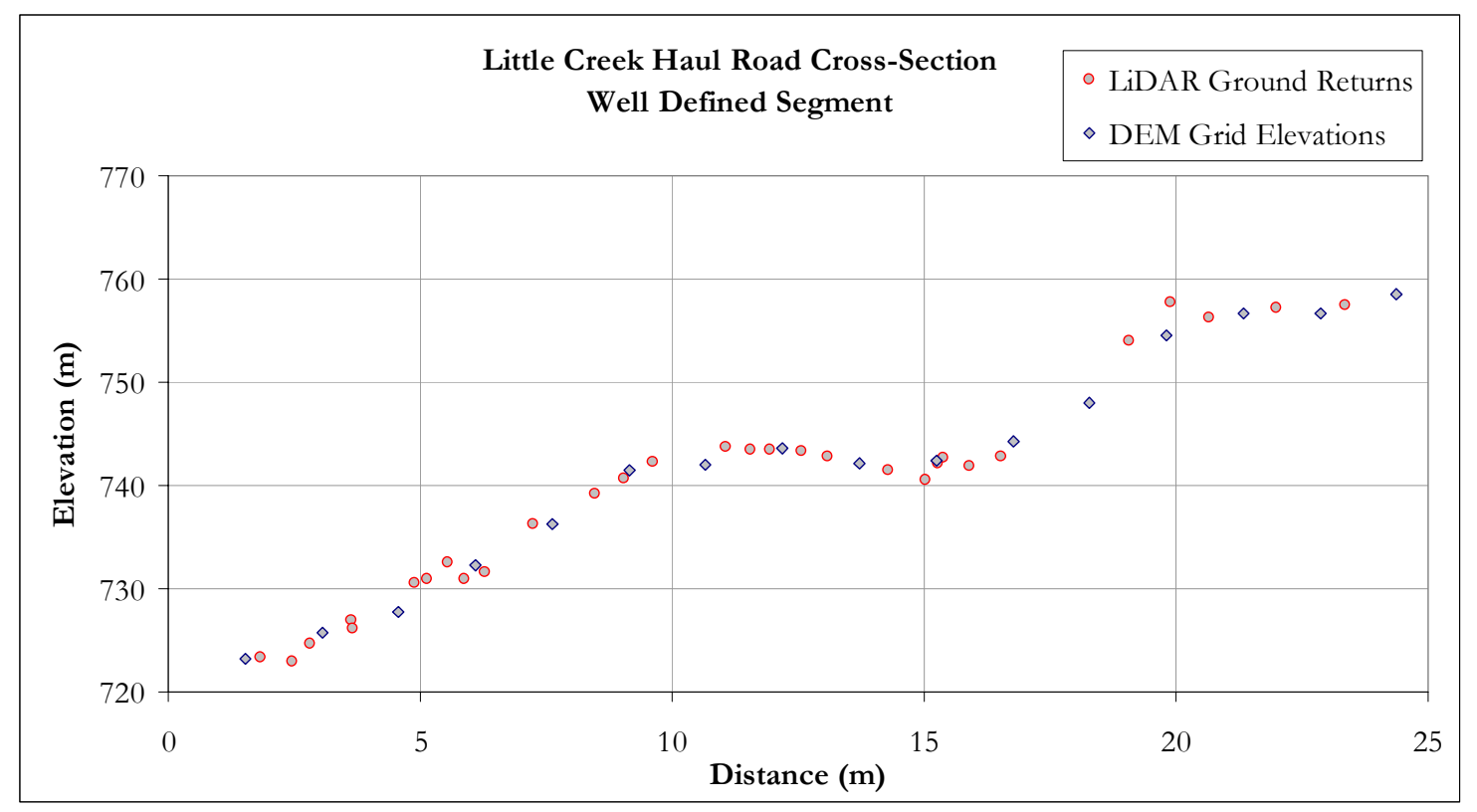

Figure 25. Cross section for a well-defined road segment.

The vertical relief for the poorly-defined road segment (Figure 26) is much more subtle than that of the well defined road. In this case, vertical relief less than $2 \mathrm{~m}$, and only this slight depression marks the road bed and outer berms. The road cross section is less than 10 meters wide and lacks significant breaks in slope. 


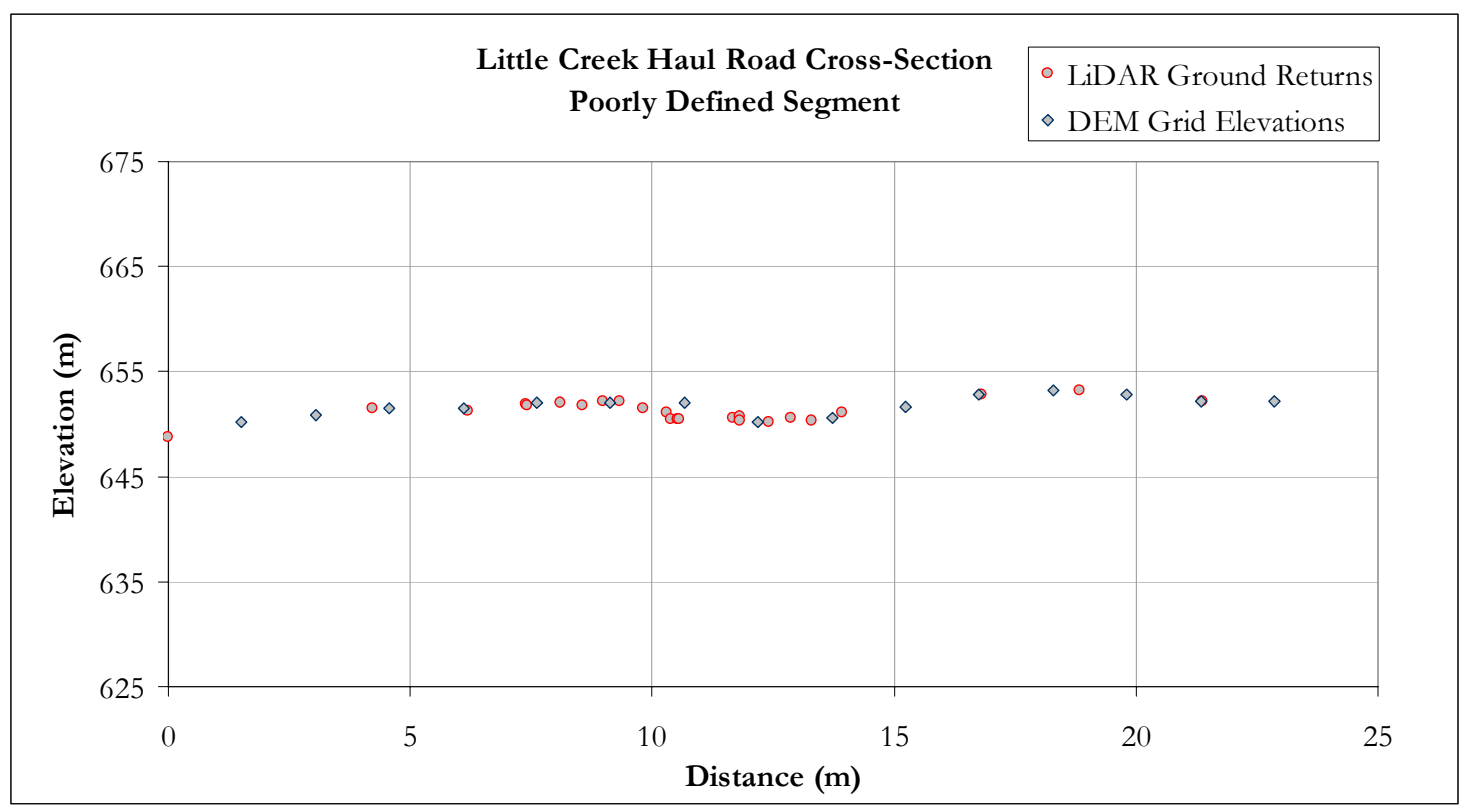

Figure 26. Cross-section for a poorly-defined road segment.

\section{$\underline{\text { Road positional error }}$}

The positional accuracy for the LiDAR-derived road was compared to the fieldsurveyed centerline through buffer overlay analysis. Result show that $95 \%$ of the digitized line length fell within $2.2 \mathrm{~m}$ or 7.2 feet normal to the true survey centerline. The results plotted in Figure 27 can be used to determine the percentage of the digitized road that is located within a certain distance of the survey centerline. For example, $90 \%$ of the digitized line falls within $1.9 \mathrm{~m}$ of the survey centerline, or a $1 \mathrm{~m}$ buffer contains $55 \%$ of the digitized road length. Positional error was also evaluated for road segments with low topographic relief, which were more difficult to identify and map. A slightly greater error occurred with the poorly defined road segment. Ninety five percent of the poorly-defined road length was contained with a $2.7 \mathrm{~m}$ buffer while ninety five percent of the well-defined road length was contained within a $2.1 \mathrm{~m}$ buffer. 


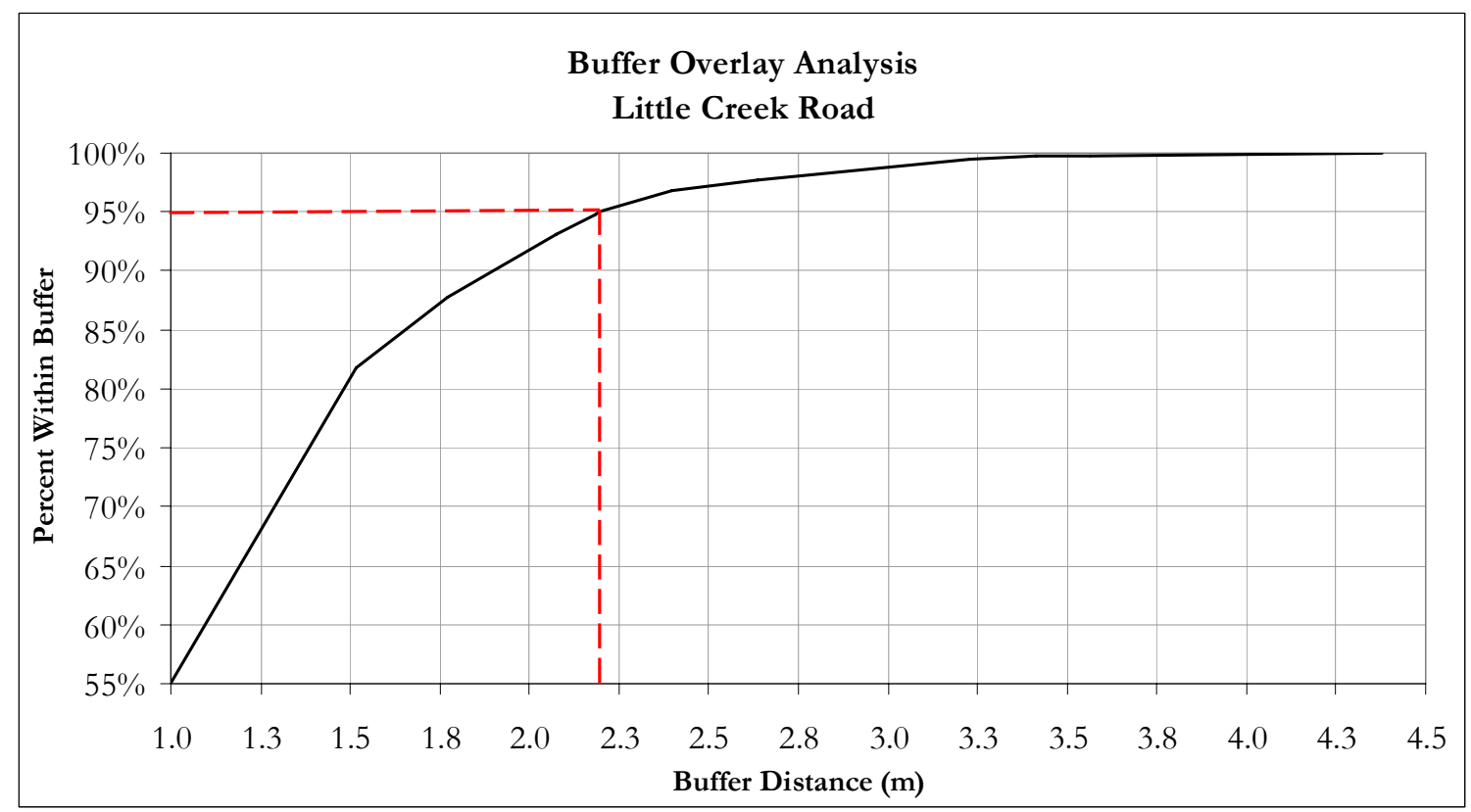

Figure 27. Positional accuracy assessment for the Little Creek road.

\section{$\underline{\text { Road slope error }}$}

LiDAR-derived road slope measurements were compared to conventional fieldsurveyed slopes. LiDAR elevations and field-surveyed elevations from 117 points along the road centerline were used to calculate 116 slope measurements. A paired t-test of matching slopes for these road segments was used to determine if the mean differences in road slope were different from zero. A p-value of 0.685 suggests failure to reject the null hypothesis, a finding that indicates that the mean difference in these paired samples is not different from zero. The $95 \%$ confidence interval for the mean difference in slope ranges from $-0.39 \%$ to $0.59 \%$. This is a very narrow range for a difference in road grade and this range includes zero, indicating that the differences between these paired slope measurements are not statistically significant. The accuracy of these matching road segments can is also evident in the longitudinal profile presented in Figure 28. 


\section{$\underline{\text { Road length }}$}

The total road length from the field-survey data was 4,006.4 meters while length from the digitized road was slightly longer at 4,020.8 meters. A difference of 14.4 meters can be expressed as a $0.4 \%$ difference from the field-measured road length or as a ratio of error of 1: 277. That is, one meter of error is encountered for every 277 meters of digitized road length. Matching road lengths can be viewed in the longitudinal profiles in Figure 28, with the road profile obtained from the ground survey in black and the profile from the digitized road feature in red.

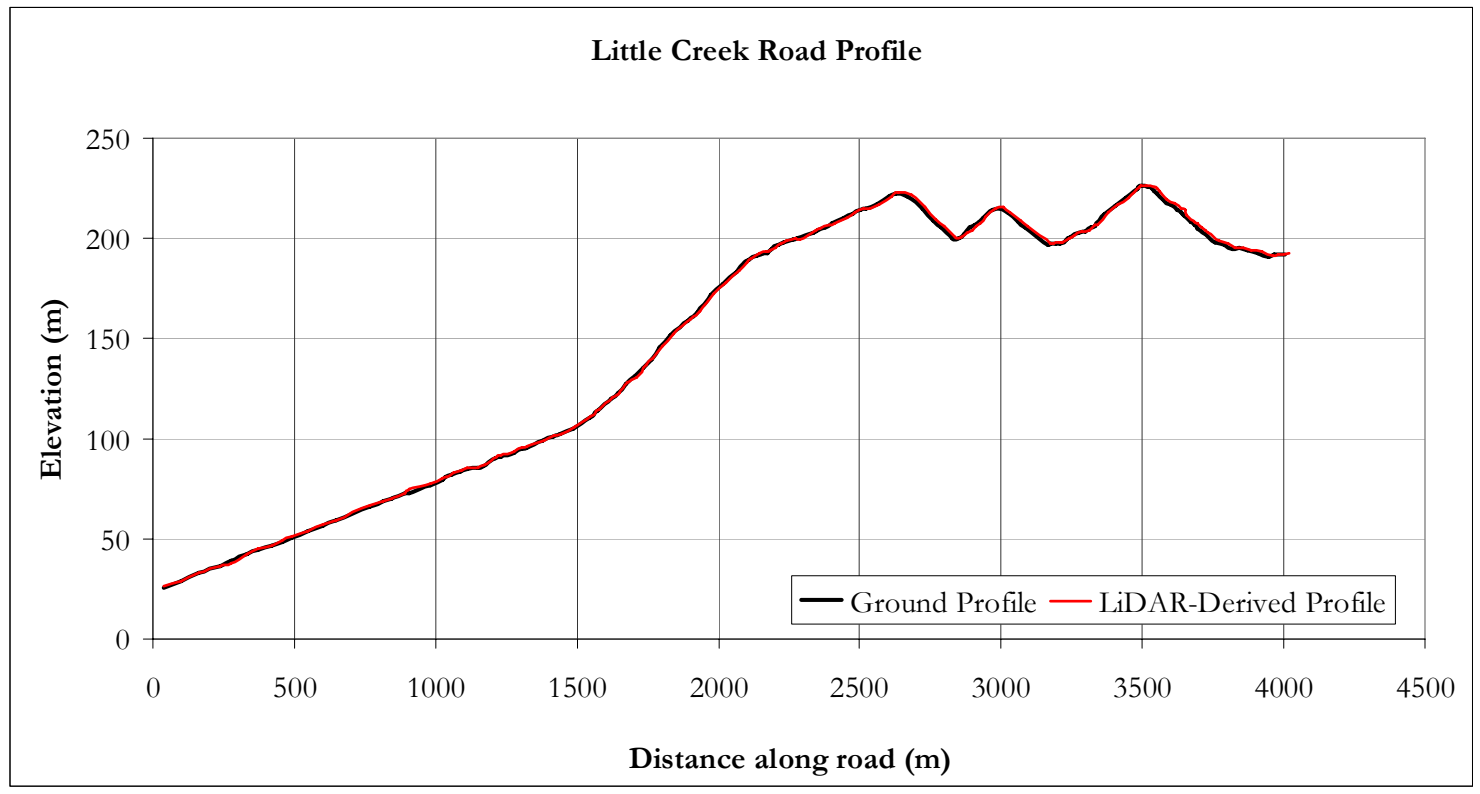

Figure 28. Visual agreement of ground survey and LiDAR-derived road profiles.

The graph illustrates the excellent agreement between the ground surveyed profile and the digitized profile. However, the discrepancy in road length more apparent in Figure 29, which illustrates a horizontal shift in the LiDAR-derived profile over the last 1,500 $\mathrm{m}$ of road length. This shift indicates the difference in road length between the two profiles, which results from the fact that the ground survey centerline and the digitized centerline follow slightly different paths along the road bed. 


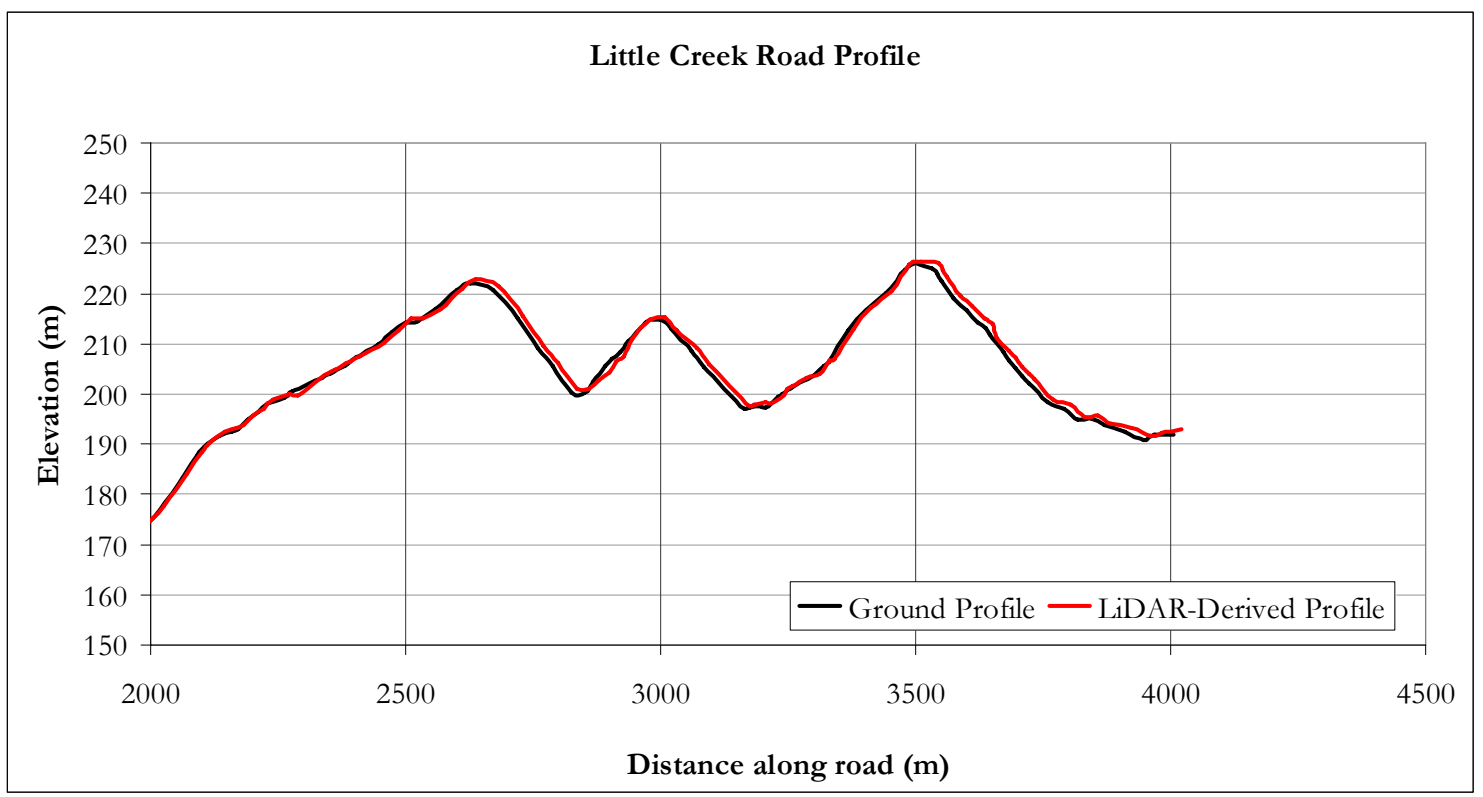

Figure 29. Detail of Little Creek road longitudinal profile and apparent horizontal shift between LiDAR-derived and ground profiles. 


\section{$\underline{\text { Stream channel positional error }}$}

Stream channel locations for Little Creek were derived with the 1.5 m LiDAR

DEM and Arc Hydro and were compared to field-surveyed channel locations at six study reaches (Figure 30).

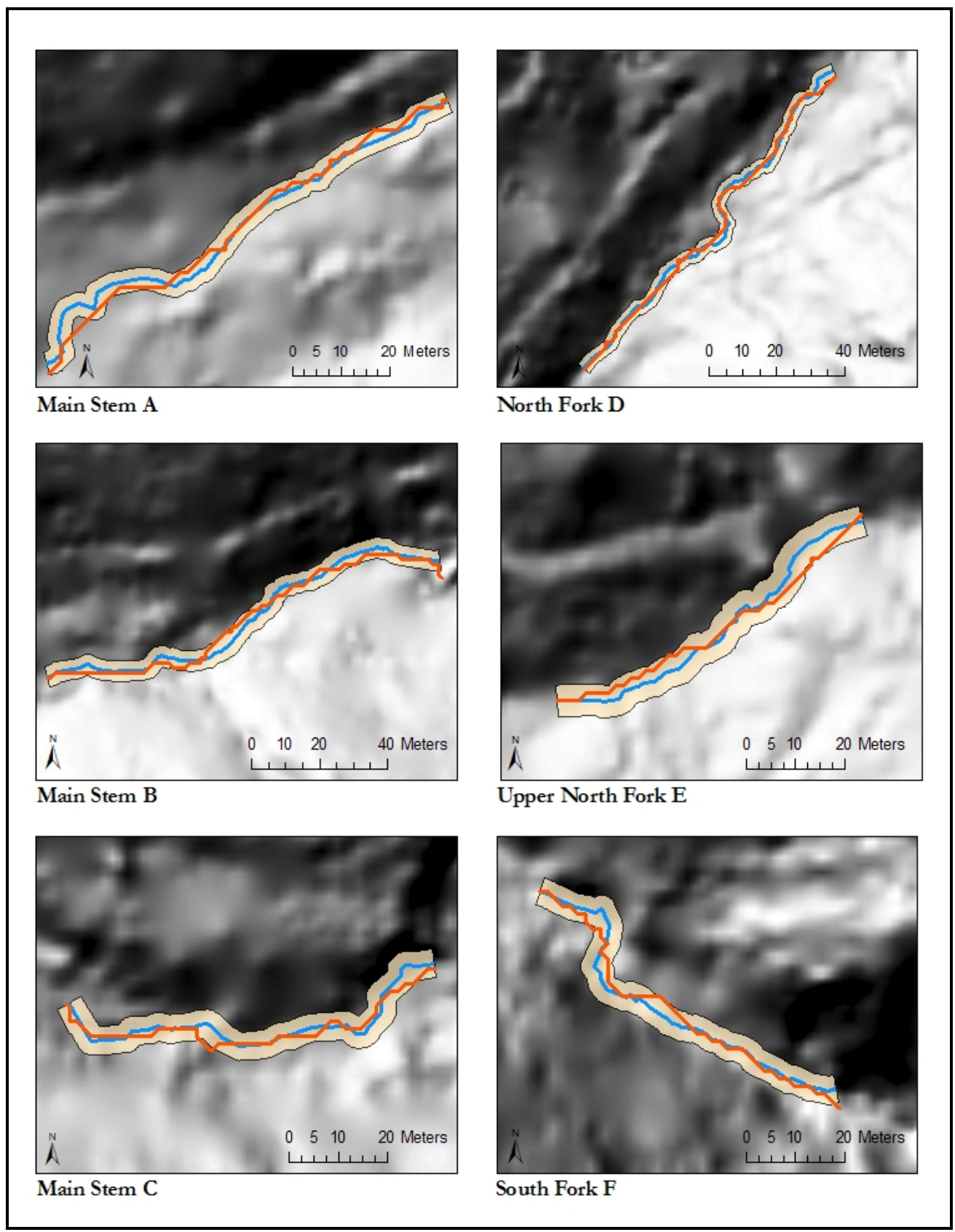

Figure 30. Field-surveyed channel position in blue, Arc Hydro defined channel in orange, and the buffer containing 95\% of the Arc Hydro channel length in beige. Note the difference in scale for North Fork D and Main Stem B. 
The average positional accuracy for the Arc Hydro-defined channel location was 2.73 meters normal to the field-surveyed thalweg. That is, $95 \%$ of the channel length delineated by Arc Hydro was located within an average $2.73 \mathrm{~m}$ to the left or right of the field-surveyed thalweg.

Table 6. Buffer distances for the six study reaches on Little Creek.

\begin{tabular}{|c|c|}
\hline $\begin{array}{l}\text { Stream } \\
\text { Reach }\end{array}$ & $\begin{array}{l}\text { Buffer } \\
\text { Distance }(\mathrm{m})\end{array}$ \\
\hline \hline A & 2.36 \\
\hline B & 3.01 \\
\hline C & 2.89 \\
\hline D & 2.05 \\
\hline E & 3.22 \\
\hline F & 2.85 \\
\hline Average & $\mathbf{2 . 7 3}$ \\
\hline
\end{tabular}

\section{$\underline{\text { Stream channel slope error }}$}

Ground-surveyed and LiDAR-derived longitudinal profiles were plotted and visually compared for each of the study reaches (Appendix B.). The profile in Figure 31 illustrates the level of agreement between the two data sources.

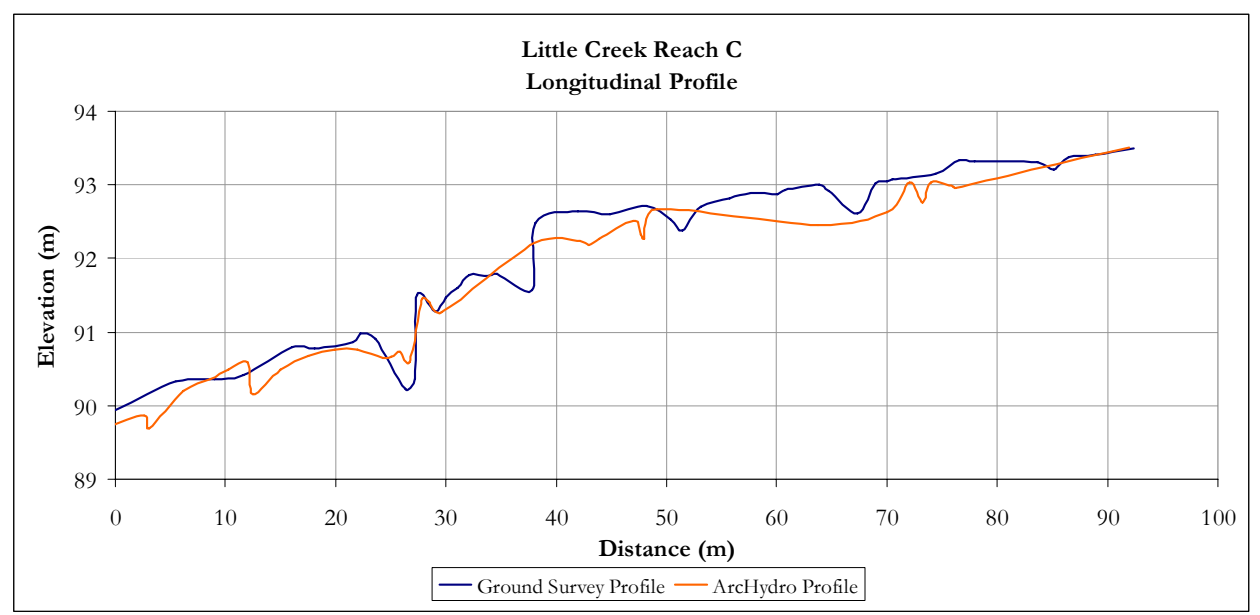

Figure 31. Longitudinal profile for Little Creek Reach C. 
While the Arc Hydro-derived profile may not capture the fine-scale undulations in bed morphology, indicating individual pool and riffle features, the LiDAR data may be capable of matching larger breaks in slope. Channel slope was calculated from each longitudinal profile on the six study reaches. The mean absolute difference in reach slope between field-survey and LiDAR-derived measurements was 0.49 percent (Table 7).

\section{$\underline{\text { Stream channel length error }}$}

Finally, stream channel length was compared to further evaluate the quality of the Arc Hydro-generated stream channel features. The Arc Hydro process resulted in stream channels that underestimated channel length on four of the six reaches while the length of two reaches was overestimated (Table 8). The discrepancy in stream channel length can be expressed as a mean absolute difference of two percent, or as an average ratio of error of 1: 110 .

Table 7. Stream longitudinal profile slope.

\begin{tabular}{|c|c|c|c|}
\hline Reach & Survey slope & LiDAR-derived slope & Difference in slope \\
\hline \hline A & $1.83 \%$ & $2.47 \%$ & $0.64 \%$ \\
\hline B & $2.21 \%$ & $2.39 \%$ & $0.18 \%$ \\
\hline C & $4.08 \%$ & $4.26 \%$ & $0.18 \%$ \\
\hline D & $5.07 \%$ & $4.93 \%$ & $-0.14 \%$ \\
\hline E & $4.81 \%$ & $3.26 \%$ & $-1.55 \%$ \\
\hline F & $4.17 \%$ & $4.42 \%$ & $0.25 \%$ \\
\hline \multirow{2}{*yy}{} & \multicolumn{3}{|c|}{ mean absolute difference } \\
\cline { 2 - 4 }
\end{tabular}

Table 8. Length of the stream channel study reaches.

\begin{tabular}{|c|c|c|c|}
\hline Reach & Survey length $(\mathrm{m})$ & LiDAR-derived length $(\mathrm{m})$ & Difference $(\mathrm{m})$ \\
\hline A & 109.28 & 108.68 & 0.60 \\
\hline B & 134.20 & 139.83 & -5.62 \\
\hline C & 92.32 & 91.98 & 0.34 \\
\hline D & 128.66 & 125.40 & 3.26 \\
\hline E & 79.98 & 78.56 & 1.42 \\
\hline F & 85.97 & 87.03 & -1.06 \\
\hline
\end{tabular}




\section{CHAPTER 7}

\section{Interpretation and Discussion}

A number of factors influence the quality and accuracy of LiDAR-derived products for mapping and describing watershed characteristics. Factors such as LiDAR system specifications, terrain and vegetation conditions, post-processing, and end-user

interpretation all contribute to the quality of end products. Findings from this study help to indicate the suitability and potential for LiDAR terrain data in resource management and assessment. Given the wide variations in vegetation conditions and LiDAR data specifications, it remains important that LiDAR data be carefully evaluated to ensure that the potential limitations of the data are fully recognized.

\section{$\underline{\text { LiDAR Ground Returns }}$}

The density of LiDAR ground returns determines the horizontal resolution of the data. This resolution indicates the size of the smallest features that can be distinguished, and also indicates the precision with which these features can be measured. For these reasons, point density is often used to characterize the quality of a LiDAR dataset. In the Little Creek watershed the density and distribution of ground returns was significantly influenced by vegetation and overstory canopy. Eighty-five percent of the LiDAR points were reflected by the canopy. The obstruction of tree canopies, particularly dense clumps of second-growth coast redwood, created gaps in the LiDAR ground return data and increased the variability in ground return spacing. Identifying the size and location of data gaps was important since these areas did not contain the same level of topographic detail as other areas. Producing a raster grid which displays the density of LiDAR ground returns is one efficient means to display these variations across the landscape. 
As was briefly outlined in Table 1, a LiDAR survey may be designed with specific flight and sensor parameters to maximize LiDAR ground returns in areas of dense vegetation. Completing the LiDAR survey during winter months when deciduous vegetation is in full leaf-off condition may increase the percentage of LiDAR pulses that reach the ground. In this study, the flight occurred on April 28, 2002 and vegetation conditions were described by the LiDAR vendor as having marginal. The emergence of red alder foliage within the study area may have resulted in reduced ground return densities especially within the riparian corridor.

Once the flight has taken place, the LiDAR filtering algorithm must correctly distinguish vegetation returns from ground returns. Oftentimes this filtering occurs within highly specialized, proprietary, algorithm used by the LiDAR vendor (Evans and Hudak, 2007). Specific details about the methods, assumptions or tolerances of the filtering are typically unknown to the end users. It is possible that improvements in the density and distribution of ground returns could be gained by applying different filtering methods. Effective filtering methods that are designed to work specifically for data in steep forested environments continue to be developed (Evans and Hudak, 2007; Kobler et al., 2007; Zaksek and Pfeifer, 2006).

\section{$\underline{\text { LiDAR Elevation Error }}$}

The vertical accuracy of LiDAR ground returns is the most commonly reported measure of LiDAR data quality. Data with high vertical accuracy can provide reliable measurements and can distinguish topographic features that have subtle vertical relief. The vertical accuracy assessment for LiDAR ground returns resulted in a RMSE of 0.29 
meters, which falls within the range of commonly reported vertical accuracy for LiDAR in mountainous conditions (Kraus and Pfeifer, 1998; Reutebuch et al., 2003). This assessment included a sufficient number of field-measured checkpoints to determine the accuracy of LiDAR elevations along the Little Creek road. An average error of $-0.19 \mathrm{~m}$ was observed among the test points. It is possible that this represents a systematic bias across the entire LiDAR dataset, though further survey work would be needed to resolve that possibility. Ground control surveys for both the LiDAR flight data and ground-based survey were conducted at different times, and using different control points. It is possible that the ground-based survey of elevations along the road centerline elevations was not accurately referenced to the stated vertical datum. These errors are typically identified with survey control data from several permanent benchmarks. For this study, control data from the road centerline survey was limited. It is possible that the surveyed elevations contain an elevation offset greater than the $0.07 \mathrm{~m}$ error identified in the control data.

Two limitations of the vertical accuracy assessment were that only a small number of sample points could be directly evaluated, and these points were not well distributed across the study area. The study area contains a wide range of terrain slope and vegetative conditions, each of which affects the accuracy of the LiDAR point data. The vertical accuracy for LiDAR points located throughout the Little Creek watershed, across the steep slopes and under various vegetation conditions remains unmeasured. Assessing the accuracy of ground returns throughout the study area would require a large number of well distributed elevation checkpoints. Such an assessment would require a substantial ground survey effort and remains outside the scope of this study. Without 
having a direct measurement, these errors could be inferred from other field-based studies in similar settings (Bater, 2008; Hodgson et al., 2004; Kraus and Pfeifer, 1998;

Reutebuch et al., 2003), or estimated from theoretical relationships (Baltsavias, 1999a).

\section{The LiDAR-Derived DEM}

The bare-earth digital elevation model is likely the most widely used form of LiDAR data. The importance of understanding uncertainty and error in DEMs is extensively documented in scientific literature (Wechsler, 2007). However, the methods used to measure or account for these uncertainties remains poorly understood by most DEM users (Wechsler, 2003).

Vertical accuracy of the DEM elevation values was evaluated with groundsurveyed elevations along the Little Creek road. Interestingly these errors were slightly smaller than the elevation errors observed with the original LiDAR points. In general, it is expected that DEM-derived elevations will have a greater error than that of the original LiDAR points. This is expected because the DEM elevations are located at a fixed horizontal spacing that is often greater than the spacing of the original LiDAR ground returns. Larger discrepancies with the DEM elevations are expected in areas of complex topography or along steep brakes in slope such as steep road cuts, where the elevation of the ground surface may change dramatically across distances smaller than the DEM resolution. This result was not observed since the ground checkpoints were located along the consistent slope of the road surface.

A jackknife validation procedure was used to quantify DEM interpolation errors relative to the LiDAR ground returns. This method was especially helpful in evaluating the differences between different interpolation methods and grid resolutions. This test 
indicated that reducing grid cell size from $3 \mathrm{~m}$ spacing to $1.5 \mathrm{~m}$ resulted in a 0.3 meter improvement in RMSE. At the $1.5 \mathrm{~m}$ resolution, the RMSE generated by different interpolation methods was also used as a criteria for selecting an appropriate interpolation method.

A more comprehensive assessment of DEM uncertainty would be a valuable addition for this study. This could be accomplished through a systematic evaluation of different grid resolutions and interpolation methods as in Bater (2008). Such an assessment would provide a more empirical basis for selecting appropriate grid size and interpolation method. Expanding the jackknife validation procedure to utilize a greater number of test and validation datasets would increase the statistical strength for evaluating RMSE. For example, a jackknife validation with 100 iterations is presented in Evans and Hudak (2007), where each iteration randomly withholds $10 \%$ of LiDAR ground returns for verification of the interpolated surface. In addition, the distribution of elevation errors introduced with LiDAR point elevations was modeled across the DEM using one thousand Monte Carlo simulations. Given the random uncertainty introduced by both LiDAR elevation error and DEM interpolation error, it was recognized that a single DEM can be considered as just one realization of the ground surface that lies within an error range of the true ground surface (Evans and Hudak, 2007). Another extensive and valuable analysis of DEM interpolation error is presented in Bater (2008), which used classification and regression tree analysis (CART) to predict and visualize DEM error based on point density, terrain slope, vegetation cover and vegetation class. This type of analysis can yield a prediction of DEM uncertainty that varies across the landscape with respect to these different ground conditions. 


\section{$\underline{\text { LiDAR-Derived Road Measurements }}$}

A surprising number of forest road and trail features were identified and mapped through visual interpretation of the $1.5 \mathrm{~m}$ DEM. The surprise was not necessarily from the fact that these features were visible, but from viewing for the first time, a thorough and detailed map of road features within the Little Creek watershed. If mapped using the aerial photo alone, the length of road features within Little Creek would be underestimated by eighty-five percent. Results from digitizing the Little Creek haul road indicate the complete road length could be mapped. However, areas of low LiDAR point density created gaps where the road was not clearly identifiable. For the purpose of manually digitizing the road position, these gaps did not present a major limitation.

If it were necessary for the DEM to depict road topography more realistically through these data gaps, then incorporating three-dimensional breaklines into the interpolation process could provide some benefits. Breaklines can be used to supplement LiDAR point data, especially for representing areas with sharp breaks in slope, such as road edges. An example of the original DEM and a DEM constructed with a road edge breakline is presented in Figure 32. In this example, the break in slope along the outside road edge was manually digitized. The elevations along this line were extracted from adjacent LiDAR ground returns. Portions of the line which did not have nearby LiDAR returns were assigned elevations according to a linear interpolation of the known elevations along the breakline. Using breakline data during the interpolation process helps to create a DEM surface that appears more realistic to the expected road topography. 


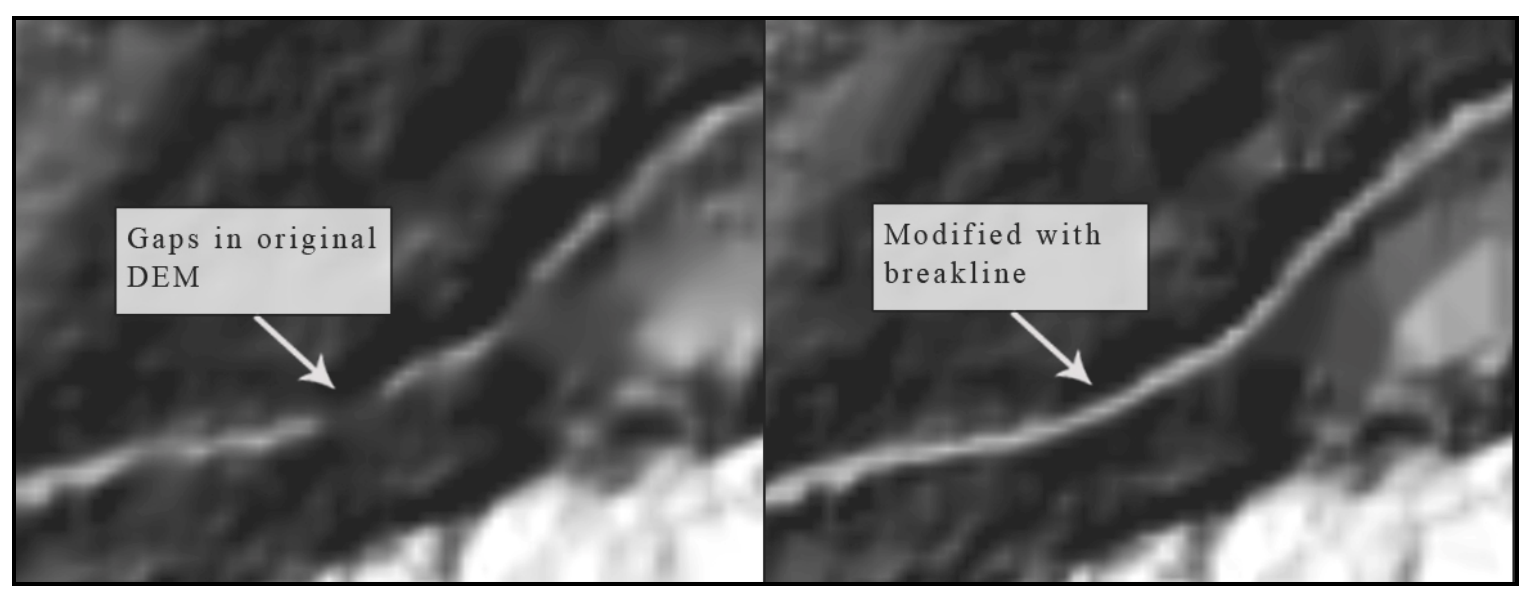

Figure 32. DEM constructed without breakline (left) and DEM constructed with breakline (right)

To a greater extent, ability to clearly identify the road was dependent on the width and vertical relief of the road cut, not necessarily the density of the LiDAR returns. In general, the Little Creek road was large enough to be readily identified on the $1.5 \mathrm{~m}$ DEM. As was presented previously in Figure 26, road features with low topographic relief were more difficult to identify.

If topographic features such as small forest roads are expected to be identified from a LiDAR DEM, then both the DEM resolution and topographic feature size should be considered. This information could also provide some useful guidelines for the initial planning of a LiDAR survey. The minimum detectable feature size may be estimated with respect to the horizontal resolution and vertical accuracy of the DEM being used. The vertical accuracy for a DEM can be expressed with the following relationship (Evans et al., 2007).

$$
R M S E_{\text {Total }}=\sqrt{\left(R M S E_{L i D A R}\right)^{2}+\left(R M S E_{D E M}\right)^{2}}
$$

$\mathrm{RMSE}_{\text {Total }}$ is a combined error that includes the uncertainty of LiDAR elevation points and the uncertainty introduced through DEM interpolation. Results for the study 
area indicate a $\mathrm{RMSE}_{\text {Total }}$ of 0.57 meters. This value could be interpreted as the vertical resolution of the DEM. As with the horizontal resolution, perhaps two times 0.57 meters or 1.14 meters serves as a minimum vertical relief for feature identification. Using this information, it is estimated that the smallest road or trail feature that can be identified using the $1.5 \mathrm{~m} \mathrm{DEM}$ is at least $3 \mathrm{~m}$ wide, with a vertical relief of 1.14 meters. A hypothetical road or trail cross section with these dimensions is illustrated in Figure 33. Additional field-based measurements of smaller roads and trail features could also help to define the minimum detectable feature size for the LiDAR DEM.

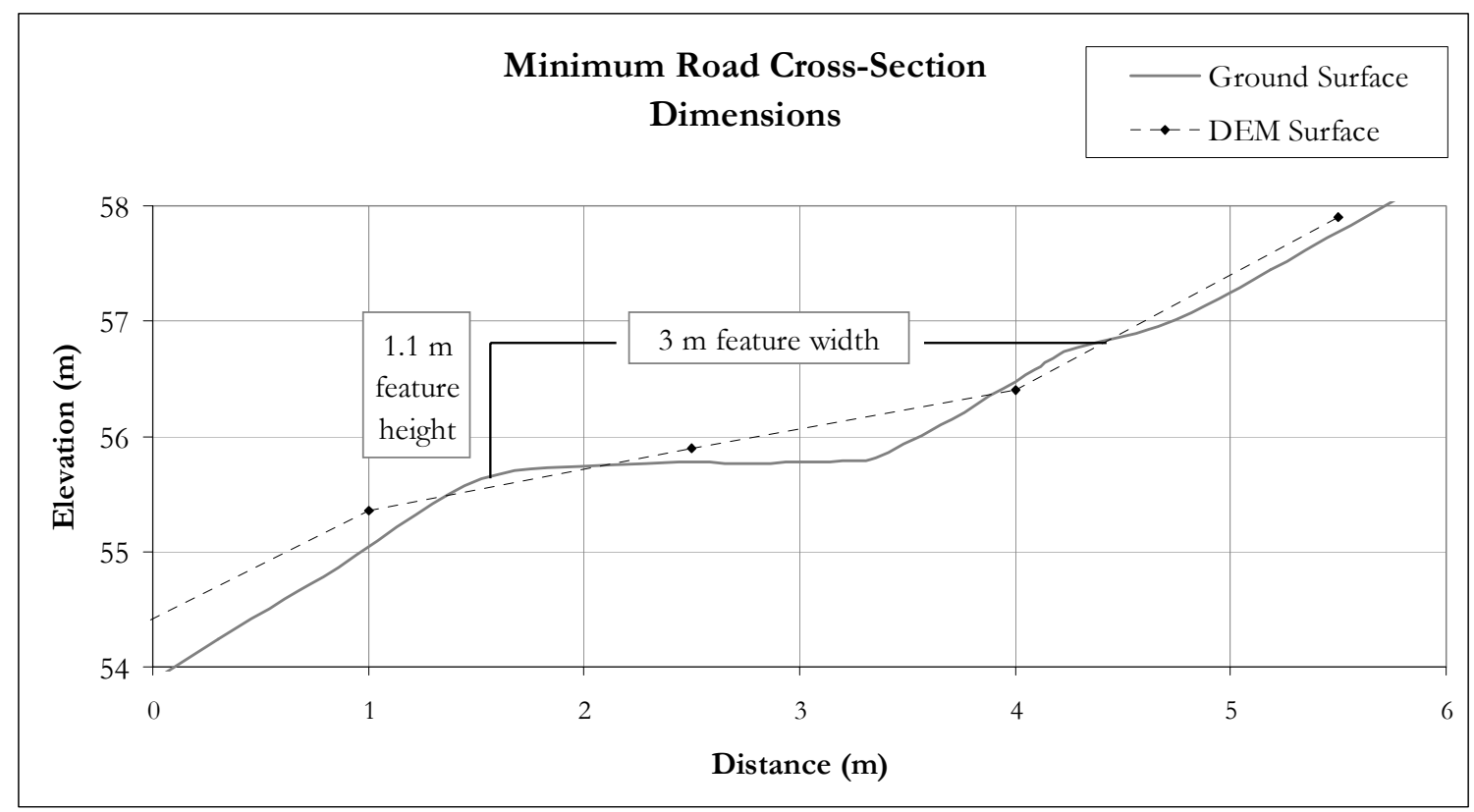

Figure 33. Hypothetical cross-section of the smallest detectable road feature.

There are few objective guidelines to judge the suitability of digitized road data for particular applications. The accuracy and completeness of road data for use in GIS is often expressed by the map scale at which the data was produced (i.e. 1: 24,000), or the map accuracy standards which it meets. For example, roads that meet the National Map 
Accuracy Standards for the 1: 24,000 scale have a horizontal accuracy of \pm 12.2 meters (Aitken and Hayes, 2006).

An objective definition for road accuracy may require that the digitized road centerline lie within the width of the true road surface. The typical driving surface width of the Little Creek haul road is designed at $3.65 \mathrm{~m}$ (12 feet). To meet this accuracy criteria, the digitized feature could deviate by one-half of the road width or $1.8 \mathrm{~m}$ on either side of the centerline to remain within the true road width. Based on the buffer analysis results presented in Figure 27 on page 62, eighty seven percent of the digitized road length would satisfy this target accuracy. This strict definition of accuracy is useful when the digitized road line will also be used to determine road slope.

Slope of the digitized road line was determined by selecting LiDAR elevations located along the digitized line. If the digitized road line were to deviate from the true road surface, these elevations would be drawn from the road cut or fill slopes. Given the steep hillslopes adjacent to the roadway, these elevations could introduce additional uncertainty into the slope calculation. In this assessment, road slope was the parameter that compared most accurately with the field-measured values. These results were achieved due to the horizontal accuracy of the digitized centerline and the relatively low vertical error of LiDAR elevations.

Finally, the length of the digitized road was compared to the ground-surveyed road length. The overestimate in road length as presented in Figure 29 on page 65 can be explained by the fact that the digitized road line and the surveyed centerline traverse slightly different paths across the road surface. Part of this discrepancy is due to the positional error incurred by visual interpretation and manual digitizing on the LiDAR- 
derived layers. In addition, however, the survey centerline is composed of straight-line segments which average 30 meters in length. In contrast, the digitized road centerline follows a smooth path across the road bed as was visible from the LiDAR derived layers. The discrepancy between these road centerlines becomes more apparent along curves in the road (Figure 34). It is possible that the straight-line segments of the ground-surveyed centerline are spaced too far apart to capture the true road sinuosity. In this case, the surveyed road length is an underestimate of true road length, which would indicate that LiDAR-derived road length might be closer to the true road length than previously presented.

As presented in the inset in Figure 30, the straight-line simplification of the ground-surveyed centerline could also have introduced an element of positional error to the "true" centerline position. The vertex points in this survey can be considered the true centerline position, but the straight-line segments that connect each vertex may deviate from the true road centerline if the road is curved. A separate analysis of positional accuracy between the centerline vertex points, and the nearest location of the LiDARderived centerline resulted in the same level of positional accuracy $(2.2 \mathrm{~m})$ at the $95^{\text {th }}$ percentile. This provides assurance that the linear segments of the ground-survey centerline do not introduce horizontal error into the assessment of LiDAR-derived positional accuracy. 


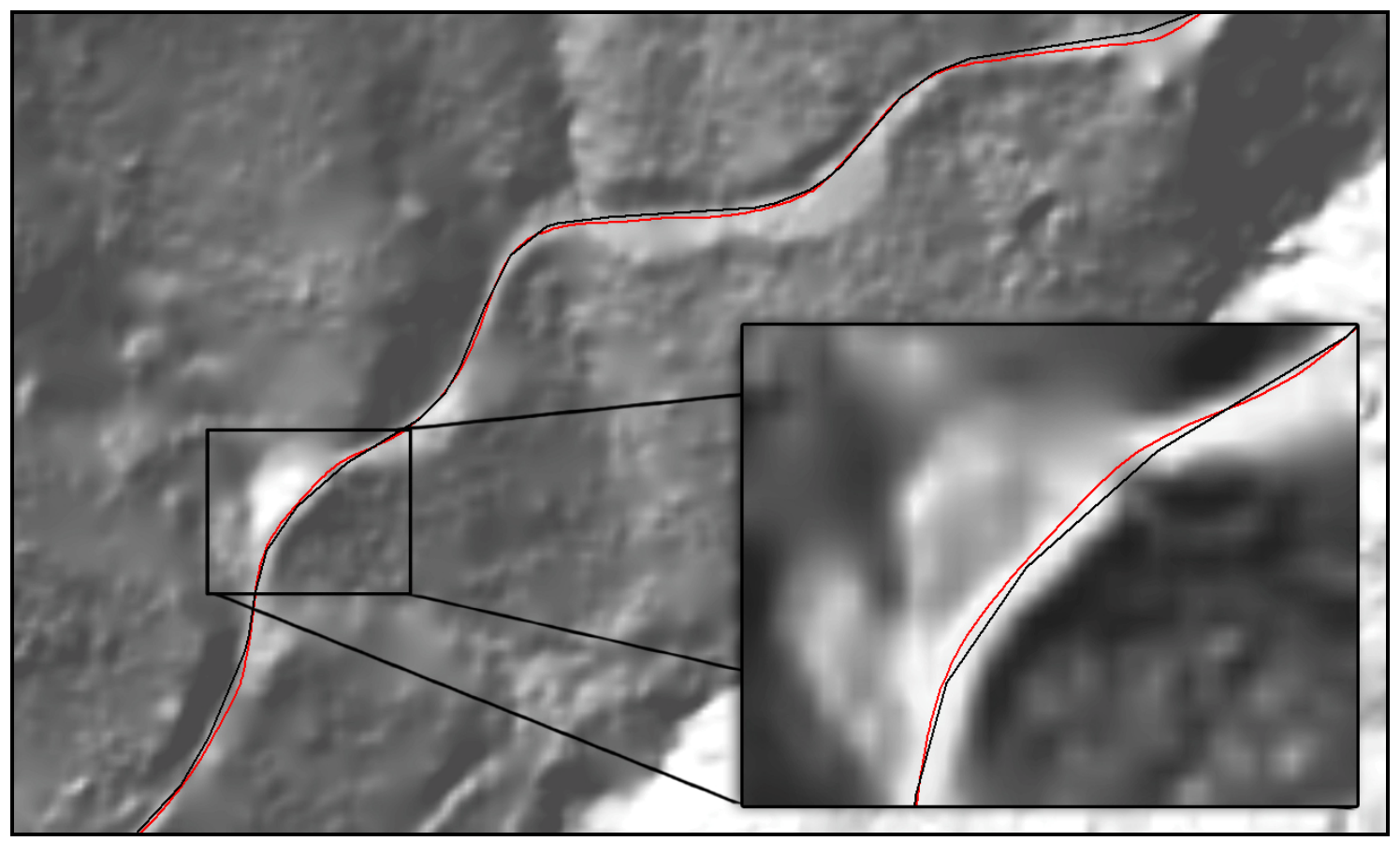

Figure 34. Road centerline from field survey (black) and digitized from DEM (red). Inset shows the angular, straight-line segments of the field survey line, compared to the smooth curve of the DEM-digitized line.

\section{$\underline{\text { LiDAR-Derived Stream Channel Measurements }}$}

The 1.5 meter DEM was used in conjunction with the Arc Hydro process to generate an accurate depiction of the Little Creek stream channel network. The average positional accuracy determined for the six study reaches was 2.7 meters normal to the surveyed thalweg. A similar level of accuracy was achieved across each of the study reaches, though the variations in accuracy could be investigated further. It is possible that LiDAR ground point density, terrain complexity and the vertical relief of the channel banks could help explain the differences in positional accuracy.

As a comparison, stream positions digitized from the USGS 1: 24,000 topographic map for the study area indicated a positional discrepancy of 15 meters from the surveyed thalweg. While the 1:24,000 map scale is not intended for detailed channel 
mapping, it is a widely used data source for many applications. The accuracy achieved in this study was higher than the accuracy achieved in similar assessments in other regions (Colson, 2006). This high level of accuracy may result from the steepness of the terrain and the use of the high-resolution DEM. The steep inner-gorge characteristics of the Little Creek watershed provide a well-defined solution for the flow direction and flow accumulation processes in Arc Hydro. When these grid-based processes are applied in areas of low topographic relief, there may is less topographic distinction of the true channel location. The precision of Arc Hydro-delineated channel is also highly dependent on grid resolution. A high-resolution DEM that accurately represents the ground surface will provide more realistic drainage characteristics. In addition, since the channel can only be positioned to the nearest grid cell, a low-resolution grid often results in an oversimplified channel pattern. As grid size decreases, the channel can be reproduced with a higher fidelity to the true channel pattern.

In terms of grid size, ninety-five percent of the channel length within the study reaches was delineated to within two grid cells on either side of the surveyed thalweg. This may again relate to the guideline that at least two grid cells is the effective minimum width for depicting linear features. In terms of ground units, the ninety-five percent of the delineated channel length was within one-half of the bankfull width of the channel. That is, the digitized line falls within the true active channel along a majority of its length. The horizontal accuracy of this derived channel is important since this centerline was used to extract the LiDAR elevations, which were in turn used to determine channel slope. 
Evaluating the slope and longitudinal profiles from each study reach provided a practical evaluation of LiDAR-derived elevation measurements. The longitudinal profiles generated from the combination of the Arc Hydro-derived channel location and LiDAR point elevations provided a reasonable agreement with the field-surveyed profile. However, from this data, it was not possible to replicate the fine-scale topography of the channel bed. This is primarily due to the lack of density and uncertainty of LiDAR elevations along the center of the channel. Extracting LiDAR elevation points that represent the channel bed and not the steep channel banks requires careful verification. Visual inspection of the extracted LiDAR elevations is recommended since it is possible for erroneously high elevation points to be extracted along the channel longitudinal profile line. Given the uncertainty in reach-scale channel slope, a LiDAR-derived profile may not be suitable for hydrologic applications that require a precise measurement of water surface slope. However, LiDAR-derived measurements may be suitable for a number of resource assessment applications. The suitability LiDAR-derived measurements depend on level of accuracy that is needed for a given application. 


\section{CHAPTER 8}

\section{Applications}

Forest road and stream channels features were accurately and efficiently mapped using the LiDAR data. The accuracy of these features was verified with respect to the field survey data, and may now aid the selection of appropriate applications of LiDAR measurements. In general, the data produced using LiDAR exhibited four valuable characteristics. First, features could be mapped thoroughly and efficiently across the entire study area. Second, features had a consistently high level of positional accuracy. Third, because of positional accuracy, these linear features were also accurate in terms of length. Finally, the horizontal and vertical accuracy of this data resulted in accurate measurements of longitudinal slope. These unique aspects can be further explored to determine suitable applications, and potential limitations of LiDAR-based mapping.

\section{$\underline{\text { High Quality Maps }}$}

The completeness and positional accuracy of LiDAR-derived GIS can be used to produce reliable, high-quality field maps for purposes ranging from recreation to timber harvest planning. In terms of traditional map accuracy standards (NMAS, 1947), the LiDAR-derived features produced in this study can be reproduced at map scales ranging up to $1: 3,000$, or $1 "=250$ feet. That is, the horizontal error of the road or stream channel features, about 2.7 meters, does not exceed 1/30" when printed at a map scale of 1: 3,000. Such maps can provide more detailed and reliable positional data than traditional sources. In addition, LiDAR-derived layers such as shaded relief or slope grids can be used as a backdrop to GIS vector data. The combination of accurate GIS 
data and high-resolution terrain data can yield clear and informative maps, and is particularly valuable for maps produced at scales larger than the traditional 1:24,000 USGS topographic quad maps (Figure 35). LiDAR-based maps can improve the efficiency of field activities by reducing positional uncertainty and can also improve the communication of mapped information among different users, including the public. The two map images in Figure 35 illustrate the difference in map detail and clarity between a traditional map and LiDAR-derived map data. 


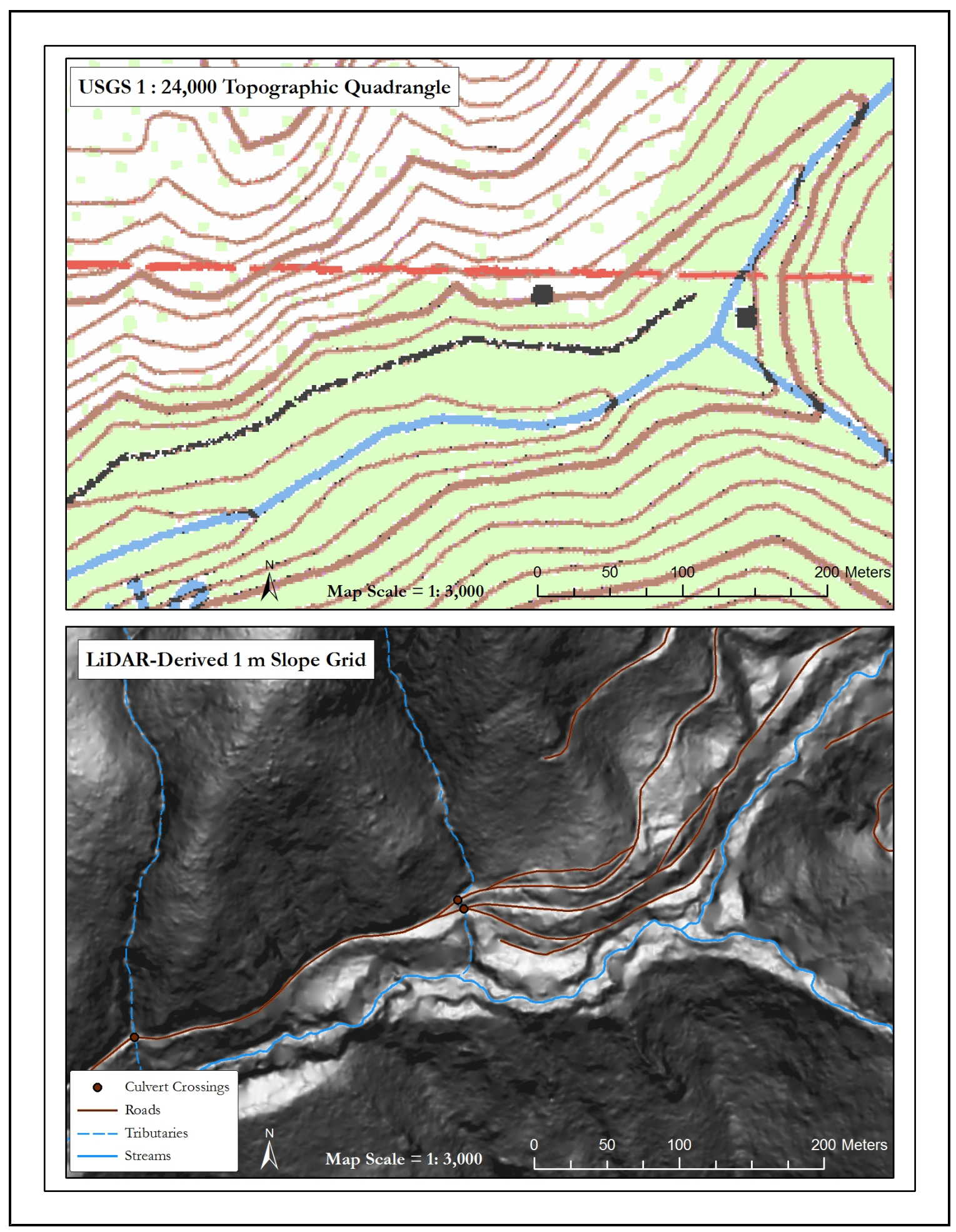

Figure 35. Map data from a traditional USGS 1: 24000 topo quad (above) and GIS data derived from a $1 \mathrm{~m} \mathrm{LiDAR} \mathrm{DEM} \mathrm{(below).}$ 


\section{$\underline{\text { Accurate Road Measurements }}$}

The ability to obtain accurate measurements of road length and slope, without the need for field surveying, can save time and improve the efficiency of project planning. Road length measurements may be required for activities such as road grading or gravel surfacing. Estimating the amount of gravel needed for a particular road requires a relatively accurate estimate of road length. In this study, the total length of the Little Creek road was measured to within $14 \mathrm{~m}$ or $0.4 \%$ of the total road length, which is well within the accuracy requirement for these planning purposes. In addition, length measurements can be obtained for any segment of the mapped road, rapidly and consistently. While LiDAR-derived measurements would be sufficiently accurate in terms of horizontal length, it may also be necessary to identify road segments with characteristics such as shallow depth to bedrock, or saturated soil conditions. Sitespecific conditions such as these cannot be readily identified from LiDAR data, and in this case may warrant field verification.

Accurate measurements of road slope are also useful for planning and road maintenance activities. For example, planning the spacing of road drainage features such as cross drains, waterbars or rolling dips is often determined by slope-class designations. The California Forest Practice Rules (CDF, 2007) indicates waterbar spacing ranging from 50 to 300 feet as determined by soil erodibility and the following slope classes; slopes less than $10 \%$, slopes from $11 \%$ to $25 \%$, slopes from $26 \%$ to $50 \%$ and road slopes greater than 50\%. LiDAR-derived slope measurements from the Little Creek road were accurate to within $0.6 \%$ of the field-surveyed slope. These measurements would be sufficient to classify road segments into these pre-defined slope classifications. Together, 
accurate measurements of road length and slope could be used to estimate the total number of drainage features that must be constructed along a typical haul road, and estimate the amount of equipment time that would be required for this activity.

\section{$\underline{\text { Accurate Stream Channel Measurements }}$}

Measurements of the stream channel network often require field-based survey methods, though some attributes can be accurately obtained from LiDAR terrain data. Channel attributes such as stream pattern, sinuosity and longitudinal slope can be useful for broad-level channel classifications, such as the Rosgen Level I stream type (Rosgen, 1994). Level I stream types provide a framework for relating the landscape with channel form and hydrologic function. For example, channels in the "A" stream type are typically located in the upland or mountainous reaches of a watershed, have steep slopes, low sinuosity, and exhibit a step-pool or cascading bed morphology. The hydrologic processes of a given reach can be better anticipated and understood by applying channel mapping and classification.

Broad Level I classification can be accomplished with the use of LiDAR terrain data. First, high-resolution terrain data can provide a detailed visualization of landscape topography, a starting point for classification. For example, different landforms or valley types are often associated with specific channel types, where broad alluvial valleys with well developed floodplains generally contain " $\mathrm{C}$ " type channels, and narrow, confined canyons of low relief typically contain "F" type channels (Rosgen, 1996). Rosgen describes such delineations using standard 1: 24,000 topographic maps and aerial photos. 
The use of LiDAR however, allows a much more detailed visualization of landscape topography, and provides accurate quantitative data to describe reach characteristics.

Comparisons from the Little Creek study reaches indicate that channel slope measured to within $0.49 \%$ of field-surveyed slope. These measurements would provide one of the necessary measurements to define the channel type. For example, many characteristics of "B" and "C" type channels are similar, but the differences in slope can distinguish between the tow channel types. Type "B" channels have a higher gradient, classified in the range of $2 \%$ to $3.9 \%$, where type "C" channels have lower slopes, generally less than $2 \%$. Here, LiDAR derived slope measurements with a precision of $0.49 \%$ could be used to distinguish " $\mathrm{B}$ " type and " $\mathrm{C}$ " type channels. Note that a single stream channel may transition from a steeper "B" type channel into a lower-relief valley to become a " $C$ " type channel downstream. Identifying the location of stream type transitions can be improved with the continuous, accurate profile measurements obtained using LiDAR.

Streams in low-relief areas are typically distinguished based on channel pattern, sinuosity and meander-width ratio (Rosgen, 1996). For stream channels in open areas, this information is extracted from aerial photo interpretation, however LiDAR terrain data can also be used, particularly for channels located under dense riparian vegetation. While the study reaches in Little Creek exhibited a low sinuosity overall, other examples are found within the surrounding Scotts Creek watershed. As presented in Figure 36, neither the $1 \mathrm{~m}$ color orthophoto or the 1: 24,000 USGS topo quad are suitable for mapping the channel pattern for Scotts Creek and Mill Creek, two channels located under 
dense overstory canopy. In contrast, the LiDAR-derived channels (blue) are sufficient to determine sinuosity and stream pattern for the Level I stream classification.

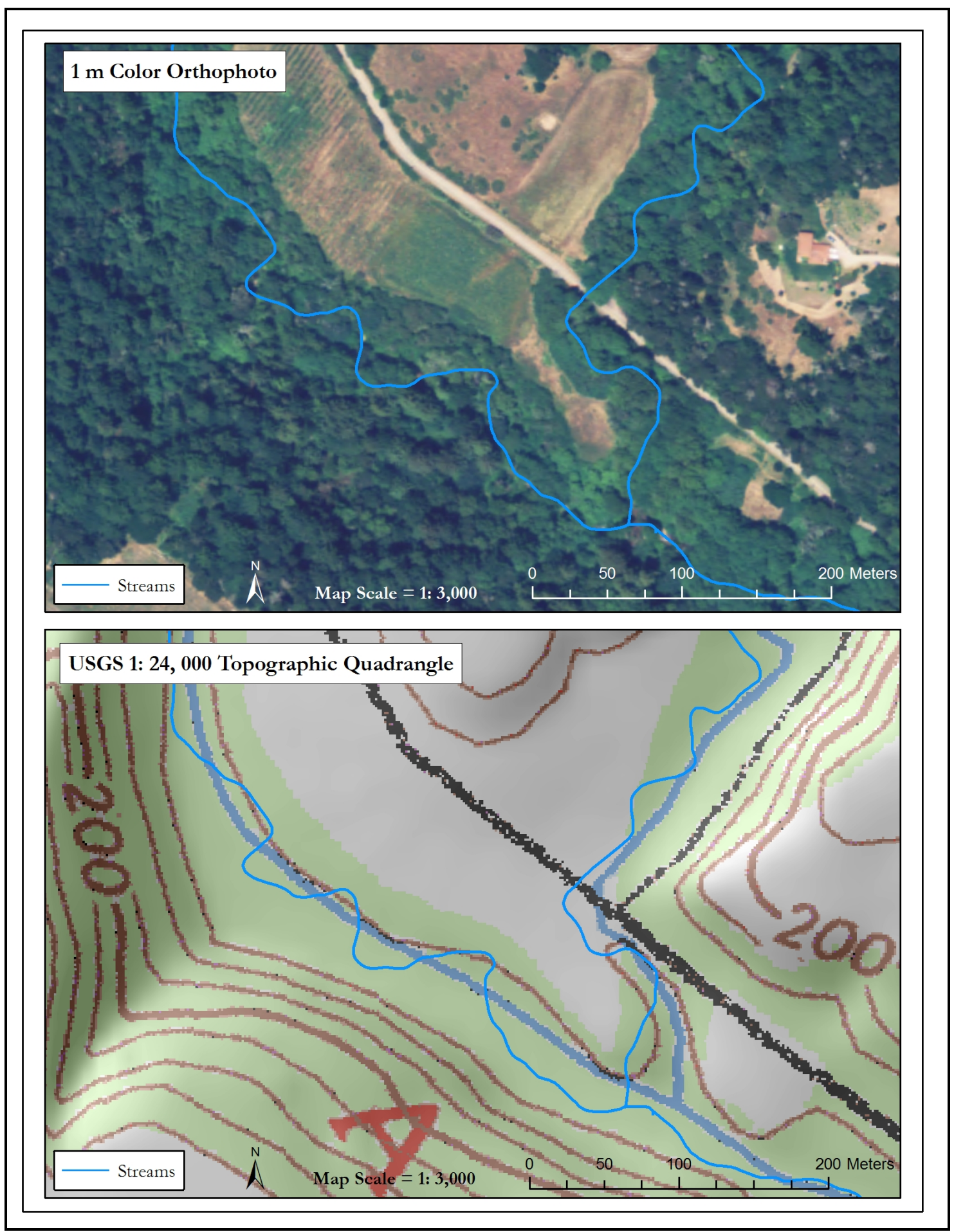

Figure 36. LiDAR-derived stream channel pattern over a $1 \mathrm{~m}$ color orthophoto and 1: 24,000 USGS topo quad, confluence of Mill Creek and Scotts Creek near Davenport, CA. 
While LiDAR-derived data provides benefits for preliminary project-level planning and reach-level classification, LiDAR datasets that are collected at larger scales, tens to hundreds of square kilometers, can also be used for watershed-wide assessments. At larger scales, LiDAR data can serve as a comprehensive and accurate dataset serving many aspects of watershed analysis.

\section{Forest Road Inventory and Assessment}

In the Little Creek watershed, analysis of the LiDAR terrain data provided a more complete inventory of forest road features compared to aerial photo interpretation. A thorough inventory of forest road locations is a valuable dataset. This data is a common prerequisite for many Cumulative Watershed Effects (CWE) assessment methods. The Equivalent Roaded Area method as modified by McGurk and Fong (1995), for example, incorporates the length of roads within $100 \mathrm{~m}$ of stream channels as one indicator of the disturbances within a watershed. Failure to identify and map these road features would yield an incomplete analysis of these road effects.

While the ERA method has often been applied to assess watershed condition, the method has two primary shortcomings (Macdonald et al., 2004). First, as an index-based approach, this method simplifies a great variety of management impacts into a single measure of disturbance (Macdonald, 2000). Second, this method is not spatially explicit, and yields little information about the locations or processes that are responsible for causing watershed impacts. For example, the effects of roads and harvesting activities are lumped into a common "equivalent" disturbance measure, regardless of the different effects that these conditions may cause (sediment impacts from roads, versus hydrologic impacts of recently-harvested areas). In addition, characteristics that vary across the 
landscape such as road slope, condition, or hydrologic connectivity to the stream channel are not adequately represented. Relatively little data is required to implement the ERA method, which contributes to its ease-of-use and to its shortcomings.

The topographic detail and measurement accuracy of LiDAR data presents an opportunity to conduct more complete, quantitative, and spatially explicit analyses of watershed impacts. For example, a number of process-based and empirically based road erosion models are used to quantify sediment production from forest roads. Such models include: WEPP:Road (Elliot and Hall, 1997), the Geomorphic Road Analysis and Inventory Package (GRAIP) (Prasad et al., 2005), Sediment Erosion and Deposition Model (SEDMODL2) (NCASI, 2003), the Washington Road Surface Erosion Model (WARSEM) (Dube et al., 2004) and others (Coe, 2006). These models produce sitespecific estimates of road erosion, though require detailed data to describe road slope, segment length, cross-sectional geometry, road surface material, vehicle traffic and underlying soil properties (USFS, 2007).

Incorporating LiDAR-based road measurements and high-resolution terrain data to model the erosion from existing road networks has not been widely explored in the literature, though this approach has been applied to evaluate new forest road designs (Aruga et al., 2005). Measurement results from the Little Creek road indicate that road parameters such as total road length, road slope, and cross-sectional geometry (Appendix C.), can be accurately obtained from LiDAR data analysis. However, other important information, such as road surface condition, evidence of rill erosion, or the locations of specific drainage features (waterbars or rolling dips) cannot be currently extracted using LiDAR data. Depending on the modeling objectives, characteristics such as waterbar 
spacing may require field identification and mapping. Nevertheless, the potential for LiDAR to provide a full inventory of road length, slope and cross sectional geometry may provide a valuable opportunity to identify and address road conditions that impose potentially adverse impacts within a given watershed.

\section{Decision Support for Road Management}

Road inventory information is also used to prioritize the decommissioning of forest roads. Decision support methods described by Madej et al., (2006) and Allison et al., (2004) incorporate road location, terrain stability and estimates of the road sediment volume that is at risk of erosion and transport. These input data are used along with complex decision-support algorithms to prioritize treatment activities. Such analyses often require field surveys in order to estimate the volume of fill material in crossings or quantify unstable road fill slopes (Madej et al., 2006). Delivery of road sediment to the stream channel must also be estimated to determine the cost or risk associated with road failure at given locations (Allison et al., 2004).

LiDAR-derived topographic profiles can be used to identify road fills that are located on steep slopes and adjacent to stream channels (Figure 37). In this example, the dashed line represents a continuation of the natural hillslope, and both the road cut and fill locations are evident. If a series of these cross-sections are extracted at intervals along a road, then it is possible to estimate the fillslope sediment volume along a road segment. A similar method of terrain analysis, visual interpretation and mapping of road cut and fill slopes from LiDAR data is presented in Haneberg, (2008). 


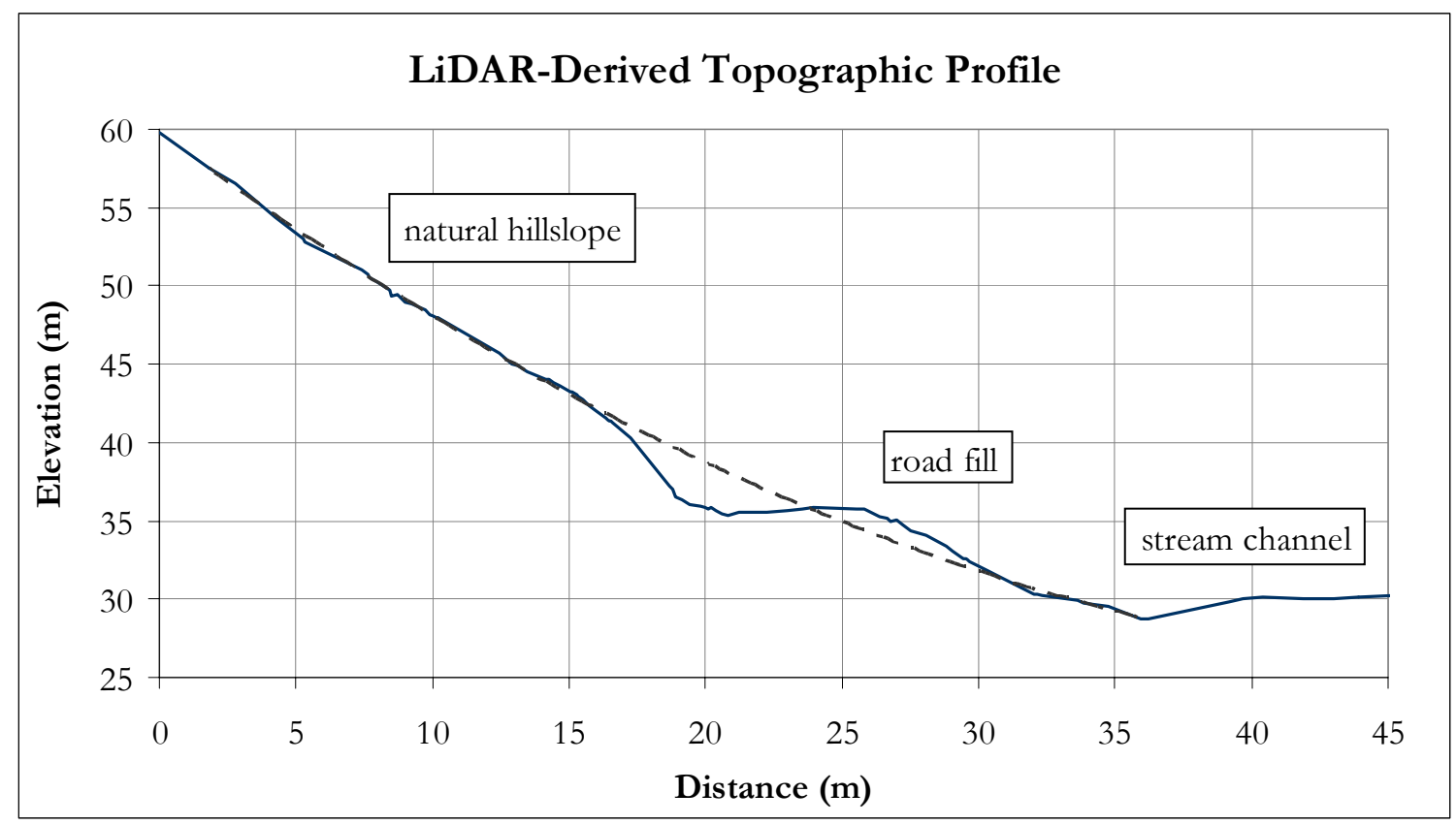

Figure 37. Hillslope, road cross-section and stream channel along a topographic profile.

LiDAR terrain data can also be used to estimate the volume of fill material that is located at road stream crossings. Fill volumes are commonly estimated based on field measurements of the channel profile, and cross-section measurements (Weaver et al., 1994). In this example, a longitudinal profile for a tributary stream (Figure 38) was extracted from the LiDAR DEM and used to depict the existing road fill conditions. This profile data was also used to simulate excavation of the fill material, which would restore the channel to its original gradient. 


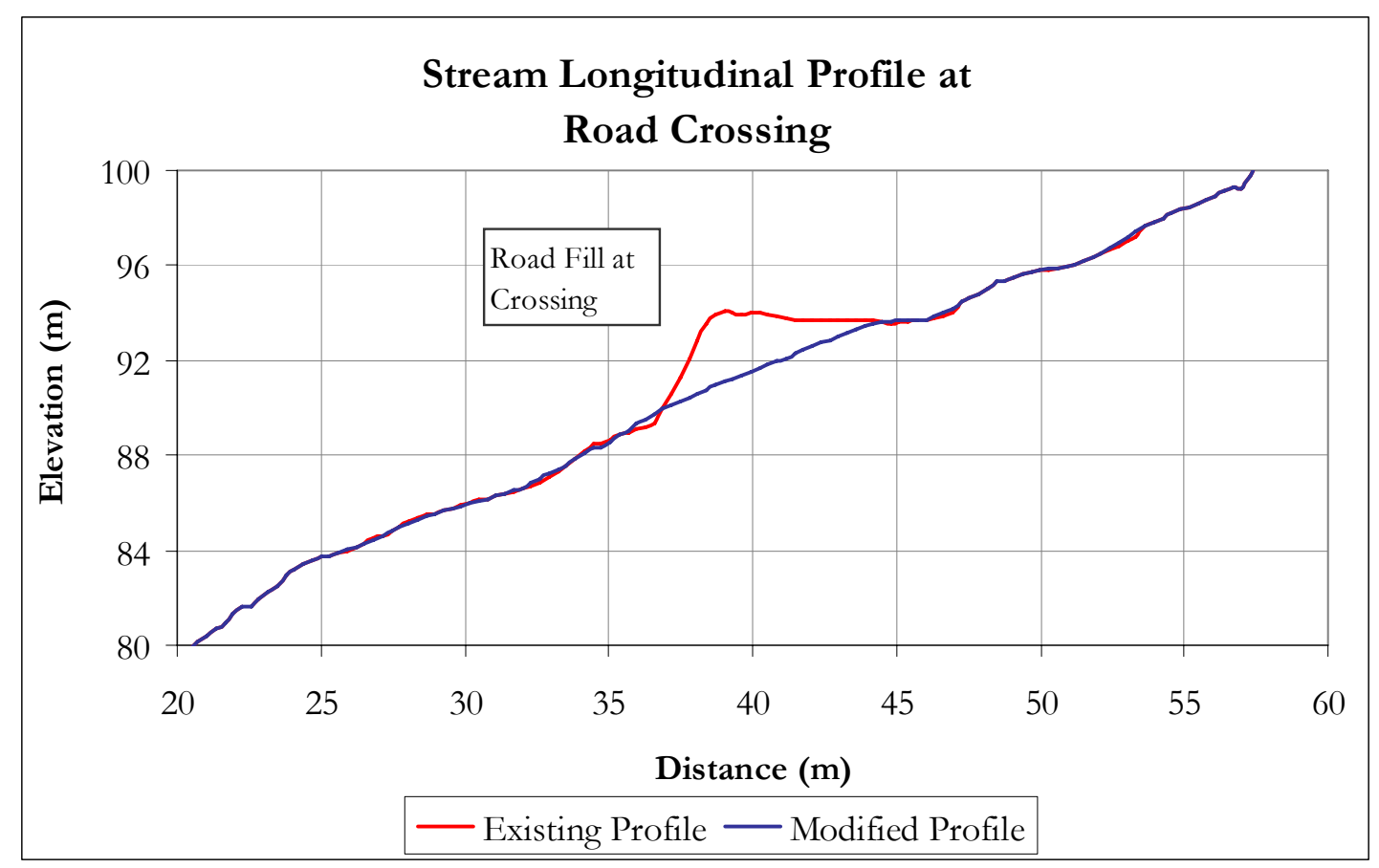

Figure 38. Longitudinal profile indicating fill material at road crossing.

Again, by extracting a series of cross-sections within the stream crossing, it is possible to estimate the volume of fill material. Longitudinal profile and cross-section data were used to create a new DEM to represent the stream channel at its natural grade, without fill material. Subtracting the actual DEM elevations from the "excavated" DEM produced a difference grid, which represents the depth of fill material. The map in Figure 39 illustrates the estimated volume of road fill at a stream crossing along the Little Creek road.

The following example application of extracting sediment volumes from LiDAR terrain data extend beyond the accuracy assessment of this study, and would require a field verification of their own. Ideally, DEM resolution and vertical accuracy could be used to determine the accuracy of volume measurements, as obtained in these examples. For now, this remains a potential application of LiDAR data, though would be a valuable focus for further study. 


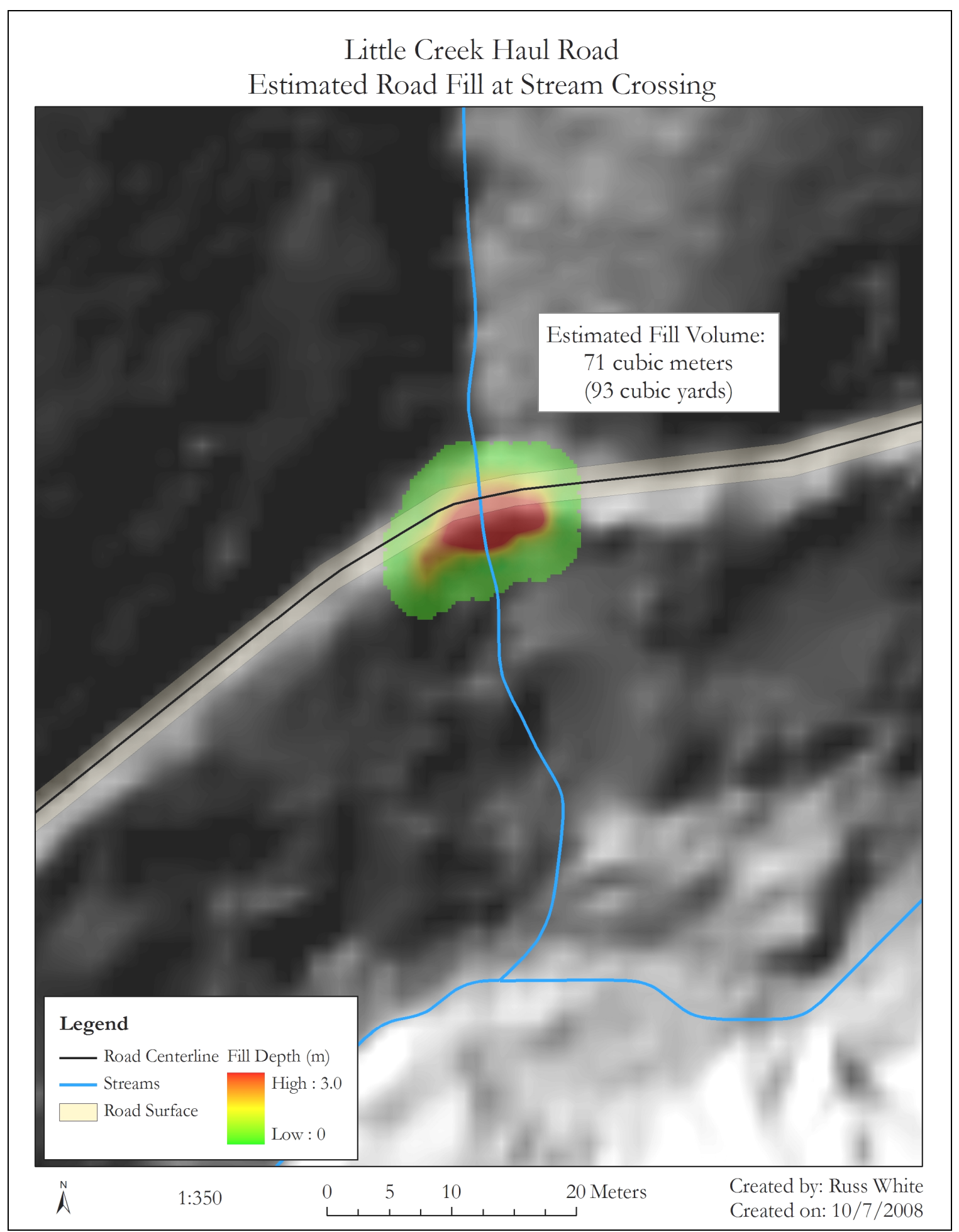

Figure 39. Road fill volume estimated from stream profile and cross section data. 


\section{Current Technology}

Over the past ten years LiDAR terrain mapping has continued to advance in capability and cost efficiency. The hardware and software components of LiDAR data production such as LiDAR sensors with higher pulse repetition frequencies, and efficient filtering algorithms are examples of continued technological improvements. Commercial software tools that can manage vast LiDAR datasets have also reduced the time and costs associated with LiDAR surveying (Maune, 2007). Compared to LiDAR surveys conducted ten or even five years ago, today, LiDAR users are able to acquire higherquality data, collected over larger areas, at a lower cost than previously possible. As presented in Table 9 these findings are supported by the on-going LiDAR research conducted at Swanton Pacific Ranch.

Table 9. LiDAR survey parameters for Swanton LiDAR flights in 2002 and 2008.

\begin{tabular}{|l|c|c|}
\hline LiDAR Survey Parameters & 2002 & 2008 \\
\hline \hline Surveyed area (ha): & 2,266 & 9,256 \\
\hline Altitude (m): & 1,828 & 853 \\
\hline Flight lines: & 13 & 12 \\
\hline Scan angle: & $25^{\circ}$ & $14^{\circ}$ \\
\hline Swath width (m): & 567 & 425 \\
\hline Swath overlap (\%): & $30 \%$ & $50 \%$ \\
\hline Pulse rate (Hz): & 20,000 & 100,000 \\
\hline Number of recorded returns: & 3 & 4 \\
\hline
\end{tabular}

In the recent 2008 survey, parameters such as lower flight altitude, narrower scan angle, greater swath width overlap and higher pulse rate, improved in the accuracy and the density of the LiDAR coverage. All LiDAR data attributes including point density, ground point spacing and the ultimate DEM resolution were improved in the 2008 LiDAR survey (Table 10). 
Table 10. LiDAR data for flights in 2002 and 2008.

\begin{tabular}{|l|c|c|}
\hline LiDAR Data Summary & 2002 & 2008 \\
\hline \hline Total point density $\left(\mathrm{pts} / \mathrm{m}^{2}\right):$ & 1.6 & 14 \\
\hline Ground point spacing $(\mathrm{m}):$ & 2.1 & 1.1 \\
\hline DEM resolution $(\mathrm{m}):$ & 1.5 & 1.0 \\
\hline Product delivery time: & 2 months & 10 days \\
\hline Survey cost: & $\$ 47,000$ & $\$ 35,000$ \\
Cost per acre: & $\$ 12.00$ & $\$ 1.60$ \\
\hline
\end{tabular}

Even with the larger project area and increased point density, the 2008 survey was still less expensive than the initial 2002 survey. The differences in price and performance can be partially explained by economies of scale (larger survey area in 2008) and the improvements in LiDAR technology and industry competition, however, the two surveys were conducted also by different firms.

A thorough side-by-side comparison of these two LiDAR datasets to evaluate differences in vertical accuracy, ground point density, and overall DEM quality would be a valuable addition to the on-going LiADR research for forested areas. The accuracy assessment of road and stream channel features as presented here, will be repeated using the updated 2008 data to quantify the improvements in LiDAR-derived products. From an initial overview, the $1 \mathrm{~m}$ resolution DEM produced from the 2008 flight is a substantial improvement over the previous $1.5 \mathrm{~m}$ DEM. The improved resolution provides sharp definition for abrupt topographic features, such as road cuts, while the overall surface is smooth and nearly free from aboveground artifacts. This smoother ground surface is achieved as a result of greater ground point coverage and, more importantly, through improved bare-earth filtering algorithms. Still, the 2008 LiDAR data exhibits some gaps in ground point data, similar to gaps observed the 2002 dataset. 
These gaps indicate that even at lower flight altitude and higher pulse frequency, some areas were still obscured by dense overstory canopy. 


\section{CHAPTER 9}

\section{Conclusions}

On-going research presented in the scientific literature continues to demonstrate the multi-purpose role of LiDAR in resource assessment and management. LiDAR has been used to find new approaches to many prevalent challenges in resource mapping. The sheer volume, accuracy, and versatility of LiDAR data offer unique capabilities compared to other remote sensing techniques. In this study, topography of the Little Creek watershed was mapped with airborne LiDAR. The resulting $1.5 \mathrm{~m}$ digital elevation model was used to obtain accurate measurements of forest roads and stream channels characteristics. Information available from LiDAR data analysis can be essential in forming improved assessments of watershed conditions and the interactions of management activities.

Despite dense canopy conditions and steep terrain characteristic of the Santa Cruz Mountains, the $1.5 \mathrm{~m}$ LiDAR DEM was suitable for the study objectives. The completeness of the Little Creek road length mapped from LiDAR indicates that typical forest road features can be identified consistently and reliably throughout the surveyed area. This level of reliability is a substantial improvement over traditional aerial photo interpretation, where only fifteen percent of the Little Creek road length could be directly mapped. Forested watersheds throughout the Santa Cruz Mountains likely contain a similar proportion of unmapped road features and unmeasured road length. This study demonstrates the ability for LiDAR terrain data to help resource managers identify, measure, and address potentially problematic road features located under dense canopy. While this capability is unique to LiDAR mapping, this application has received very 
little documentation among other LiDAR studies. Acquiring road inventory data for watershed assessment and forest management may provide added justification for future wide-area LiDAR surveys.

The accuracy of both the forest road and stream channel features derived from LiDAR can provide for quantitative and site-specific analysis, without the need for ground-based surveys. The positional accuracy of forest road features, $2.2 \mathrm{~m}$, and stream channels, $2.7 \mathrm{~m}$, is well suited for producing high-quality maps that represent true on-theground conditions. The horizontal positional error in mapping these features did not exceed to the actual width of these features on the ground. Field maps produced with LiDAR-derived features will reduce the uncertainty of traditional map data sources, improve navigation in the field, and provide better communication of field conditions among resource professionals.

In addition to positional accuracy, other attributes such as feature length and longitudinal slope can be reliably obtained, extending the potential for many types of GIS-based analyses. Extracting accurate road length and slope measurements from LiDAR data my improve the efficiency and scale of road erosion modeling. Similarly, the ability to obtain accurate measurements stream channel slope, sinuosity, and local morphology provide important insights to understanding hydrologic functioning. While field surveys may be used to collect this type of data, LiDAR offers the ability to extract this information at broad scales, where field surveying is not feasible. Finally, in addition to the vector-based GIS features derived from LiDAR data, the highly detailed shadedrelief and slope grids, used as background layers, add immensely to understanding the 
overall landscape. These LiDAR-derived layers provide an important physical context that adds to understanding and interpreting other geospatial data.

This study demonstrates the value of obtaining high-quality terrain data in areas where traditional remote sensing and field survey approaches are insufficient. There are new opportunities to improve the scope, efficiency, and consistency in mapping roads and stream channel features. The methods presented in this study represent basic and straightforward examples of a growing number of LiDAR applications in resource management. It is becoming clear that airborne LiDAR holds tremendous potential to provide accurate, detailed, and broad-scale support for multiple resource assessments in diverse and complex environments. 


\section{Literature Cited}

Aitken, M. J., \& Hayes, J. L. (2006). Roads in landscape modeling: A case study of a road data layer and use in the interior northwest landscape analysis system (PNW-RN-552). Portland, OR: USDA Forest Service, Pacific Northwest Research Station.

Akay, A. E. (2004). A new method of designing forest roads. Turk. J. Agric. For., 28, 273-279.

Allison, C., Sidle, R. C., \& Tait, D. (2004). Application of decision analysis to forest road deactivation in unstable terrain. Environmental Management, 33(2), 173-185.

Andersen, H. E., Reutebuch, S. E., \& McGaughey, R. J. (2006). A rigorous assessment of tree height measurement obtained using airborne LiDAR and conventional field methods. Can. J. For. Res., 32(5), 355-366.

Aruga, K., Sessions, J., \& Miyata, E. S. (2005). Forest road design with soil sediment evaluation using a high resolution DEM. J For Res, 10, 471-479.

Asner, G. P., Keller, M., Pereira, R., \& Zweede, J. (2002). Remote sensing of selective logging in Amazonia assessing limitations based on detailed field observations, Landsat ETM+, and textural analysis. Remote Sensing of Environment, 80, 483496.

Baltsavias, E. P. (1999a). Airborne laser scanning: basic relations and formulas. Photogrammetric Engineering and Remote Sensing, 54, 199-214.

Baltsavias, E. P. (1999b). Airborne laser scanning: existing systems and firms and other resources. Photogrammetric Engineering and Remote Sensing, 54, 164-198.

Bater, C., W. (2008). Assessing indicators of forest sustainability using LiDAR remote sensing. Unpublished Master's Thesis, University of British Columbia, Vancouver, BC.

Bowen, Z. H., \& Waltermire, R. G. (2002). Evaluation of light detection and ranging (LiDAR) for measuring river corridor topography. Journal of the American Water Resources Association, 38(1), 33-41.

Brardinoni, F., Slaymaker, O., \& Hassan, M. A. (2003). Landslide inventory in a rugged forested watershed: a comparison between air-photo and field survey data. Geomorphology, 54, 179-196.

California Forest Practice Rules, (2007). California Department of Forestry and Fire Protection, Title 14, California Code of Regulations, Chapters 4, 4.5, and 10, Sacramento, CA. 
Carson, W., \& Reutebuch, S. E. (1997, April 7-10). A rigorous test of USGS digital elevation models in forested areas of Oregon and Washington. Paper presented at the 1997 ACSM/ASPRS Annual Convention, Seattle, WA.

Carson, W. W., Andersen, H. -E., Reutebuch, S. E., \& McGaughey, R. J. (2004). LiDAR applications in forestry - an overview. Paper presented at the American Society of Photogrammetry and Remote Sensing Annual Conference, Denver, CO.

Charlton, M. E., Large, A. R. G., \& Fuller, I. C. (2003). Application of airborne LiDAR in river environments: The river Coquet, Northumberland, UK. Earth Surface Processes and Landforms, 28, 299-306.

Clode, S., Kootsookos, P., \& Rottensteiner, F. (2004). The automatic extraction of roads from LiDAR data. Paper presented at the ISPRS WG III/3.

Clode, S., Rottensteiner, F., Kootsookos, P., \& Zelniker, E. (2007). Detection and vectorization of roads from LiDAR data. Photogrammetric Engineering and Remote Sensing, 73(5), 517-536.

Coe, D. B. R. (2006). Sediment production and delivery from forest roads in the Sierra Nevada, California. Unpublished Master's Thesis, Colorado State University, Fort Collins, CO.

Colson, T. P. (2006). Stream network delineation from high-resolution digital elevation models. Unpublished Ph.D., North Carolina State University, Raleigh, North Carolina.

Coops, N. C., Hilker, T., Wulder, M. A., St-Onge, B., Newnham, G., Siggens, A., et al. (2007). Estimating canopy structure of Douglas-fir forest stands from discretereturn LiDAR. Trees: Structure and Function, 21(3), 295-310.

Crow, P., Benham, S., Devereux, B. J., \& Amable, G. S. (2007). Woodland vegetation and its implications for archaeological survey using LiDAR. Forestry, 80, 241252.

Dube, K. V., Megahan, W. F., \& McCalmon, M. (2004). Washington road surface erosion model. Olympia, WA: Washington Department of Natural Resources.

Elliot, W. J., \& Hall, D. E. (1997). Water erosion prediction project (WEPP) forest applications. Retrieved from: http://forest.moscowfsl.wsu.edu/fswepp/

Espinoza, F., \& Owens, R. E. (2007). Identifying roads and trails hidden under canopy using LiDAR. Unpublished Master's Thesis, Naval Postgraduate School, Monterey, CA. 
ESRI. (2006a). Arc hydro tools overview (Version 1.2). Redlands, CA: Environmental Systems Research Institute.

ESRI. (2006b). ArcMap (Version Version 9.2). Redlands, CA: Environmental Systems Research Institute.

Evans, J. S., \& Hudak, T. (2007). A multiscale curvature algorithm for classifying discrete return LiDAR in forested environments. IEEE Transactions on Geoscience and Remote Sensing, 45(4), 1029-1038.

Flood, M. (2001). LiDAR activities and research priorities in the commercial sector. International Archives of Photogrammetry and Remote Sensing, 34-3/W4.

Flood, M., \& Gutelius, B. (1997). Commercial implications of topographic terrain mapping using scanning airborne laser radar. Photogrammetric Engineering and Remote Sensing, 63(4), 327-329,363-366.

Gaedeke, M. C. (2006). Preharvest calibration of the little creek watershed a paired and nested watershed analysis. Unpublished Master's Thesis, California Polytechnic State University, San Luis Obispo.

Garcia, V. C. (2004). Using GIS and LiDAR to map the headwaters stream networks in the piedmont ecoregion of North Carolina. Unpublished Master's Thesis, North Carolina State University, Raleigh, NC.

Glenn, N. F., Streutker, D. R., Chadwick, D. J., Thackray, G. D., \& Dorsch, S. J. (2006). Analysis of LiDAR-derived topographic information for characterizing and differentiating landslide morphology and activity. Geomorphology, 73, 131-148.

Goodchild, M. F., \& Hunter, G. J. (1997). A simple positional accuracy measure for linear features. Geographical Information Science, 11(3), 299-306.

Haneberg, W. C. (2008). High-resolution LiDAR-based landslide hazard mapping and modeling, UCSF Parnassus Campus, San Francisco, USA. Seattle, WA: Haneberg Geosciences.

Hengl, T. (2006). Finding the right pixel size. Computers \& Geosciences, 32, 1283-1298.

Hickman, J. C. (1993). The Jepson Manual: higher plants of California. Berkeley, CA: University of California Press.

Hodgson, M. E., \& Bresnahan, P. (2004). Accuracy of airborne LiDAR-derived elevation: Empirical assessment and error budget. Photogrammetric Engineering and Remote Sensing, 70(3), 331-339. 
$\mathrm{Hu}$, Y. (2003). Automated extraction of digital terrain models, roads and buildings using airborne LiDAR data. Unpublished Ph.D., University of Calgary, Calgary.

Hutchinson, M. F. (1989). A new procedure for gridding elevation and stream line data with automatic removal of spurious pits. Journal of Hydrology, 106, 211-232.

Hyyppä, H. (2005). Factors affecting the quality of DTM generation in forested areas. Paper presented at the ISPRS WG III/3, III/4, V3 Workshop "Laser scanning $2005 "$.

Hyyppä, J., Pyysalo, U., Hyyppä, H., \& Samberg, A. (2000). Elevation accuracy of laser scanning-derived digital terrain and target models in forest environment. Paper presented at the EARSeL-SIG-Workshop LIDAR, Dresden/FRG.

James, L. A., Watson, D. G., \& Hansen, W. F. (2007). Using LiDAR data to map gullies and headwater streams under forest canopy: South Carolina, USA. Catena, 71, 132-144.

Jazouli, R., Verbyla, D. L., \& Murphy, D. L. (1994). Evaluation of SPOT panchromatic digital imagery for updating road locations in a harvested forest area.

Photogrammetric Engineering and Remote Sensing, 60(12), 1449-1452.

Jenks, G. (1981). Lines, computers, and human frailties. Annals of the Association of American Geographers, 71(1), 1-10.

Jones, A. F., Brewer, P. A., Johnstone, E., \& Macklin, M. G. (in press). High-resolution interpretative geomorphological mapping of river valley environments using airborne LiDAR data. Earth Surface Processes and Landforms.

Keim, R., Skaugset, A., \& Bateman, D. S. (1999). Digital terrain modeling of a small stream channel with a total-station theodolite. Advances in Water Resources, 23, 41-48.

Kobler, A., Pfeifer, N., Ogrinc, P., Todorovski, L., Ostir, K., \& Dzeroski, S. (2007). Repetitive interpolation: A robust algorithm for DTM generation from aerial laser scanner data in forested terrain. Remote Sensing of Environment, 108, 9-23.

Kraus, K., \& Pfeifer, N. (1998). Determination of terrain models in wooded areas with airborne laser scanner data. Journal of Photogrammetry and Remote Sensing, 53, 193-203.

Krogstad, F., \& Schiess, P. (2004). The allure and pitfalls of using LiDAR topography in harvest and road design. Paper presented at the International Mountain Logging Conference. 
Laptev, I., Mayer, H., Lindeberg, T., Eckstein, W., Steger, C., \& Baumgarter, A. (2000). Automatic extraction of roads from aerial images based on scale space and snakes. Machine Vision and Applications, 12, 23-31.

Lee, H., Slatton, C. K., \& Jhee, H. (2005, July, 2005). Detecting forest trails occluded by dense canopies using ALSM data. Paper presented at the Geoscience and remote sensing symposium, Seoul, South Korea.

Lefsky, M. A., Cohen, W. B., Parker, G. G., \& Harding, D. J. (2002). LiDAR remote sensing for ecosystem studies. BioScience, 52(1), 19-30.

Lim, K., Treitz, P., Wulder, M., St-Onge, B., \& Flood, M. (2003). Remote sensing forest structure. Progress in Physical Geography, 27(1), 88-106.

Liu, X. (2008). Airborne LiDAR for DEM generation: some critical issues. Progress in Physical Geography, 32(1), 31-49.

Liu, X., Zhang, Z., Peterson, J., \& Shobhit, C. (2007). The effect of LiDAR data density on DEM accuracy. Paper presented at the MODSIM07 International Congress on Modeling and Simulation.

Macdonald, L. (2000). Evaluating and Managing Cumulative Effects: Processes and Constraints. Environmental Management, 26(3), 299-315.

Macdonald, L., Coe, D. B. R., \& Litschert, S. (2004). Assessing cumulative watershed effects in the central Sierra Nevada: hillslope measurements and catchment-scale modeling. Arcata, CA: Pacific Southwest Research Station.

Madej, M. A., Eschenbach, E. A., Diaz, C., Teasley, R., \& Baker, K. (2006). Optimization strategies for sediment reduction practices on roads in steep, forested terrain. Earth Surface Processes and Landforms, 31, 1643-1656.

Maune, D. F. (2007). Digital elevation model technologies and applications: The DEM users manual, 2nd edition (2nd ed.). Bethesda, MD: American Society of Photogrammetry and Remote Sensing.

McGurk, B. J., \& Fong, D. R. (1995). Equivalent roaded area as a measure of cumulative effect of logging Environmental Management, 19(4), 609-621.

McKean, J., \& Roering, J. (2005). Operational accuracy of LiDAR in mountainous terrain. Boise, ID: USDA Forest Service Rocky Mountain Research Station.

Mena, J. B. (2003). State of the art in automatic road extraction for GIS update. Pattern Recognition Letters, 24, 3037-3058. 
Metternicht, G., Hurni, L., \& Gogu, R. (2005). Remote sensing of landslides: An analysis of the potential contribution to geo-spatial systems for hazard assessment in mountainous environments. Remote Sensing of Environment, 98, 284-303.

Miller, S. N., Shrestha, S. R., \& Semmens, D. (2004, May 23-28). Semi-automated Eextraction and validation of channel morphology from LIDAR and IFSAR terrain data. Paper presented at the ASPRS Annual Conference, Denver, CO.

Mouton, A. (2005). Generating stream maps using LiDAR derived digital elevation models and 10-m USGS DEM. Unpublished Master's Thesis, University of Washington, Seattle, WA.

Murphy, P. N. C., Ogilive, J., Meng, F. R., \& Arp, P. (2007). Stream network modeling using LiDAR and photogrammetric digital elevation models: A comparison and field verification. Hydrologic Processes, 1-8.

Naesset, E. (2004). Practical large-scale forest stand inventory using small-footprint airborne scanning laser. Scandinavian Journal of Forest Research, 19(2), 164179.

NCASI. (2003). SEDMODL 2 technical documentation. Retrieved October 5, 2008, from http://www.ncasi.org/Support/Downloads/Detail.aspx?id=5

Nelson, P. A., Smith, J. A., \& Miller, A. J. (2006). Evolution of channel morphology and hydrologic response in an urbanizing drainage basin. Earth Surface Processes and Landforms, 31, 1063-1079.

Opitz, D. W., Rao, R., \& Blundell, J. S. (2006). Automated 3-D feature extraction from terrestrial and airborne LiDAR. Paper presented at the 1st International Conference on Object-based Image Analysis (OBIA).

Pereira, L. M. G., \& Wicherson, R. J. (1999). Suitability of laser data for deriving geographical information a case study in the context of management of fluvial zones. Journal of Photogrammetry and Remote Sensing, 54, 105-114.

Pike, R. J. (2002). A bibliography of terrain modeling (geomorphometry), the quantitative representation of topography. Menlo Park, CA.

Prasad, A., Tarboton, D. G., Luce, C. H., \& Black, T. A. (2005, July). A GIS tool to analyze forest road sediment production and stream impacts. Paper presented at the 25th ESRI Users Conference, San Diego, CA.

Renslow, M. C. (2000). Evaluation of multi-return LIDAR for forestry applications. Salt Lake City, UT: Remote Sensing Applications Center, United States Forest Service. 
Reutebuch, S. E., Andersen, H.-E., \& McGaughey, R. J. (2005). Light detection and ranging (LIDAR): An emerging tool for multiple resource inventory. Journal of Forestry, 103(6), 286-292.

Reutebuch, S. E., McGaughey, R. J., Andersen, H. E., \& Carson, W. (2003). Accuracy of a high-resolution LiDAR terrain model under a conifer forest canopy. Can. J. For. Res., 29(5), 527-535.

Rieger, W., Kerschner, M., Reiter, T., \& Rottensteiner, F. (1999). Roads and buildings from laser scanner data within a forest enterprise. Paper presented at the ISPRS Commission III WG/2.

Riitters, K., Wickham, J., \& Coulston, J. (2004). Use of road maps in national assessment of forest fragmentation in the United States. Ecology and Society, 9(2), 13.

Ritchie, J. C. (1996). Remote sensing applications to hydrology: airborne laser altimeters. Hydrologic Sciences, 41(4), 625-636.

Robison, E. G., Mills, K., Paul, J., Dent, L., \& Skaugset, A. (1999). Storm Impacts and Landslides of 1996: Final Report. Corvallis, OR: Oregon Department of Forestry.

Rosgen, D. L. (1994). A classification of natural rivers. Catena, 22, 169-199.

Rosgen, D. L. (1996). Applied river morphology. Pagosa Springs, CO: Wildland Hydrology.

Saleh, R. (2004). Special issue on linear feature extraction from remote sensing data for road network delineation and revision. Photogrammetric Engineering and Remote Sensing, 70(12).

Sawyer, J. O., \& Keeler-Wolf, T. (1995). A manual of California vegetation. Sacramento, CA: California Native Plant Society.

Schulz, W. H. (2007). Landslides susceptibility revealed by LiDAR imagery and historical records, Seattle, Washington. Engineering Geology, 89, 67-87.

Sessions, J., Abdullah, A., Murphy, G., Chung, W., \& Aruga, K. (2006). Road and harvesting planning and operations. In G. Shao \& K. M. Reynolds (Eds.), Computer Applications in Sustainable Forest Management (pp. 83-99): Springer.

Shamayleh, H., \& Khattak, A. (2003). Utilization of LiDAR Technology for Highway Inventory. Paper presented at the 2003 Mid-Continent Transportation Research Symposium Ames, Iowa.

Shannon, C. E. (1949). Communication in the presence of noise. Proceedings of the IEEE, 86(2), 447-457. 
Shrestha, R. L., Carter, W. E., Lee, M., Finer, P., \& Satori, M. (1999). Airborne laser swath mapping: accuracy assessment for surveying and mapping applications. Surveying and Land Information Systems, 59(2), 83-94.

Sithole, G., \& Vosselman, F. (2004). Experimental comparison of filter algorithms for bare-earth extraction from airborne laser scanning point clouds. Journal of Photogrammetry and Remote Sensing, 59, 85-101.

Snyder, N. P., \& Schultz, L. L. (2005). Stream morphologic measurements from airborne laser swath mapping: comparisons with filed surveys, traditional DEMs and aerial photographs. Paper presented at the American Geophysical Union.

Souleyrette, R., Hallmark, S., Pattnaik, S., O'Brien, M., \& Veneziano, D. (2003). Grade and cross slope estimation from LiDAR-based surface models. Ames, IA: Midwest Transportation Consortium.

Strahler, A., Quantitative geomorphology of drainage basins and channel networks. In Handbook of Applied Hydrology, Chow, Editor; McGraw-Hill: New York, 1964.

Tarboton, D. G. (1997). A new method for determination of flow directions and contributing areas in grid digital elevation models. Water Resources Research, 33(2), 309-319.

Tarolli, P., \& Tarboton, D. G. (2006). A new method for determination of most likely landslide initiation points and the evaluation of digital terrain model scale in terrain stability mapping. Hydrology and Earth System Sciences, 10, 663-677.

Tenenbaum, D. E., Band, L. E., Kenworthy, S. T., \& Tague, C. L. (2006). Analysis of soil moisture patterns in forested and suburban catchments in Baltimore, Maryland, using high-resolution photogrammetric and LiDAR digital elevation datasets. Hydrologic Processes, 20, 219-240.

USFS. (2001). Forest roads: A synthesis of scientific information. Corvallis, Or: USDA Forest Service Pacific Northwest Research Station.

USFS. (2007). WEPP:Road WEPP forest road erosion predictor. Retrieved 10/5/08, 2008, from http://forest.moscowfsl.wsu.edu/cgi-bin/fswepp/wr/wepproad.pl.

USGS. (1992). Standards for digital elevation models: United States Geological Survey.

Van Den Eeckhaut, M., Poessen, J., Verstraeten, G., Vanacker, V., Nyssen, J., Moeyersons, J., et al. (2007). Use of LiDAR-derived images for mapping old landslides under forest. Earth Surface Processes and Landforms, 32, 754-769.

Vianello, A., Cavalli, M., \& Tarolli, P. (2009). LiDAR-derived slopes for headwater channel network analysis. Catena, 76, 97-106. 
Weaver, W. E., \& Hagans, D. K. (1994). Handbook for forest and ranch roads. Arcata, CA: Pacific Watershed Associates.

Wechsler, S. P. (2003). Perceptions of digital elevation model uncertainty by DEM users. URISA Journal, 15(2), 8.

Wechsler, S. P. (2007). Uncertainties associated with digital elevation models for hydrologic applications: a review. Hydrology and Earth System Sciences, 11, 1481-1500.

West, J. A. (2009). [Traversing swanton road]. Unpublished data.

Woodget, A. S., Donoghue, D. N. M., \& Carbonneau, P. (2007). An assessment of airborne LiDAR for forest growth studies. Ekscentar, 10, 47-52.

Yu, X., Hyyppä, J., Kaartinen, H., \& Maltamo, M. (2004). Automatic detection of harvested trees and determination of forest growth using airborne laser scanning. Remote Sensing of Environment, 90, 451-462.

Zaksek, K., \& Pfeifer, N. (2006). An improved morphological filter for selecting relief points from a LiDAR point cloud in steep areas with dense vegetation. Delft, The Netherlands: Delft Institute of Earth Observation and Space systems 


\section{APPENDIX A.}

Python Script for Buffer Overlay Analysis in ArcMap 


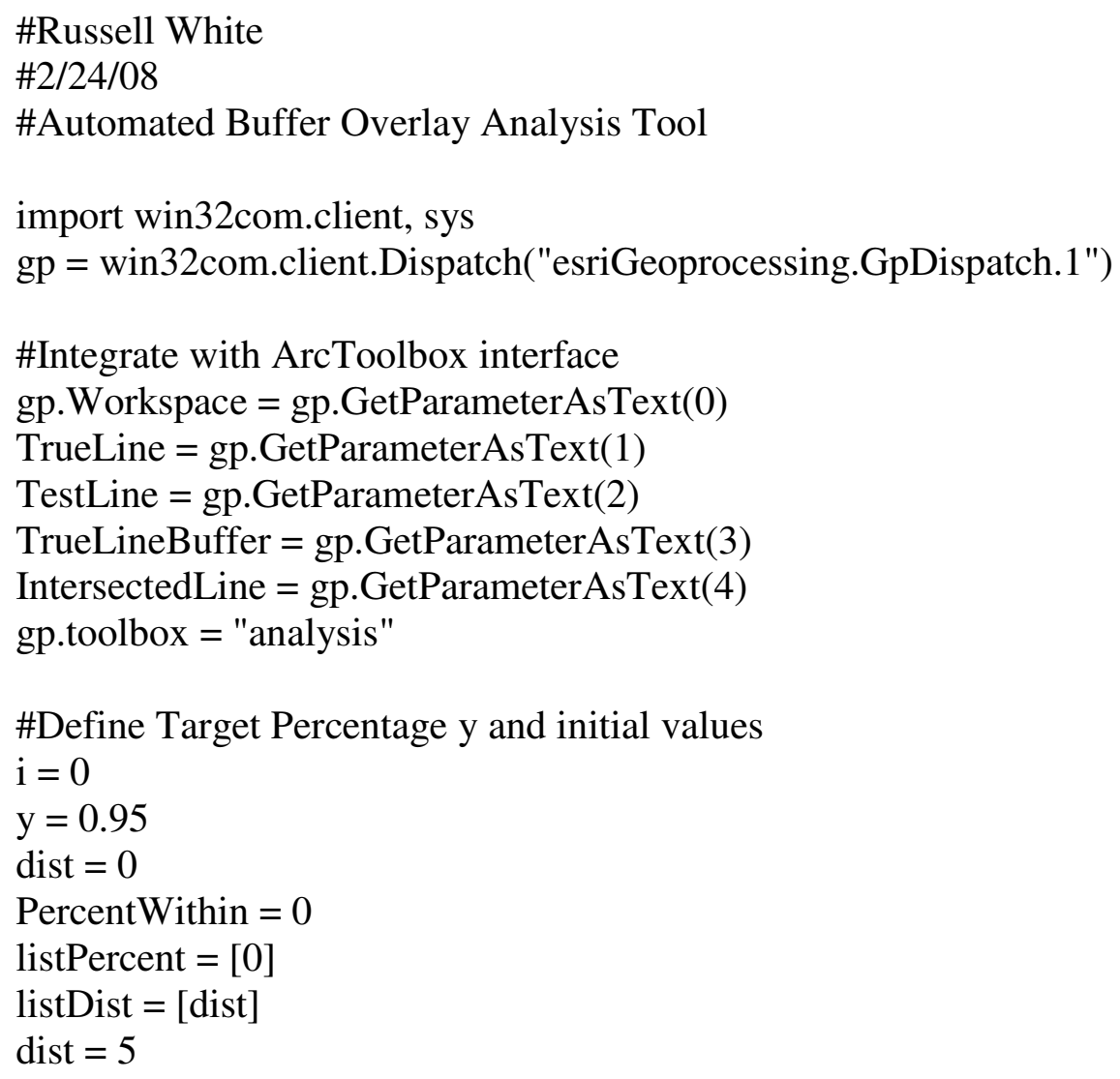

\#Iterative process to terminate as PercentWithin approaches y while PercentWithin < $(\mathrm{y}-0.001)$ :

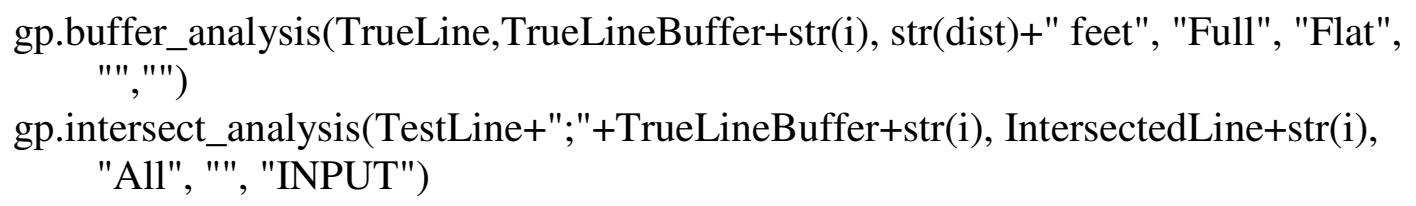

\#Get the Length of the TestFeature

updCurs $=$ gp.UpdateCursor(TestLine)

row $=$ updCurs.Next()

while row:

Shape_Length = row.GetValue $($ "Shape_Length")

row $=$ updCurs.Next()

TestLength $=$ Shape $\_$Length

\#Get the updated length of testline within the buffer updCurs $=$ gp.UpdateCursor(IntersectedLine+str(i)) row $=$ updCurs.Next() while row:

Shape_Length = row.GetValue("Shape_Length") row $=$ updCurs.Next () 
\#Percentage within

PercentWithin $=$ Shape_Length/TestLength

$\mathrm{i}=\mathrm{i}+1$

\#Append current percent to dictionary

listDist.append(dist)

listPercent.append(PercentWithin)

\#Determine the buffer distance for the next iteration

dist $=(((y-$ listPercent $[\mathrm{i}-1]) *($ dist-listDist $[\mathrm{i}-1])) /($ PercentWithin-listPercent $[\mathrm{i}-$

1]))+listDist $[\mathrm{i}-1]$ 


\section{APPENDIX B.}

Stream Channel Longitudinal Profiles at Six Study Reaches 


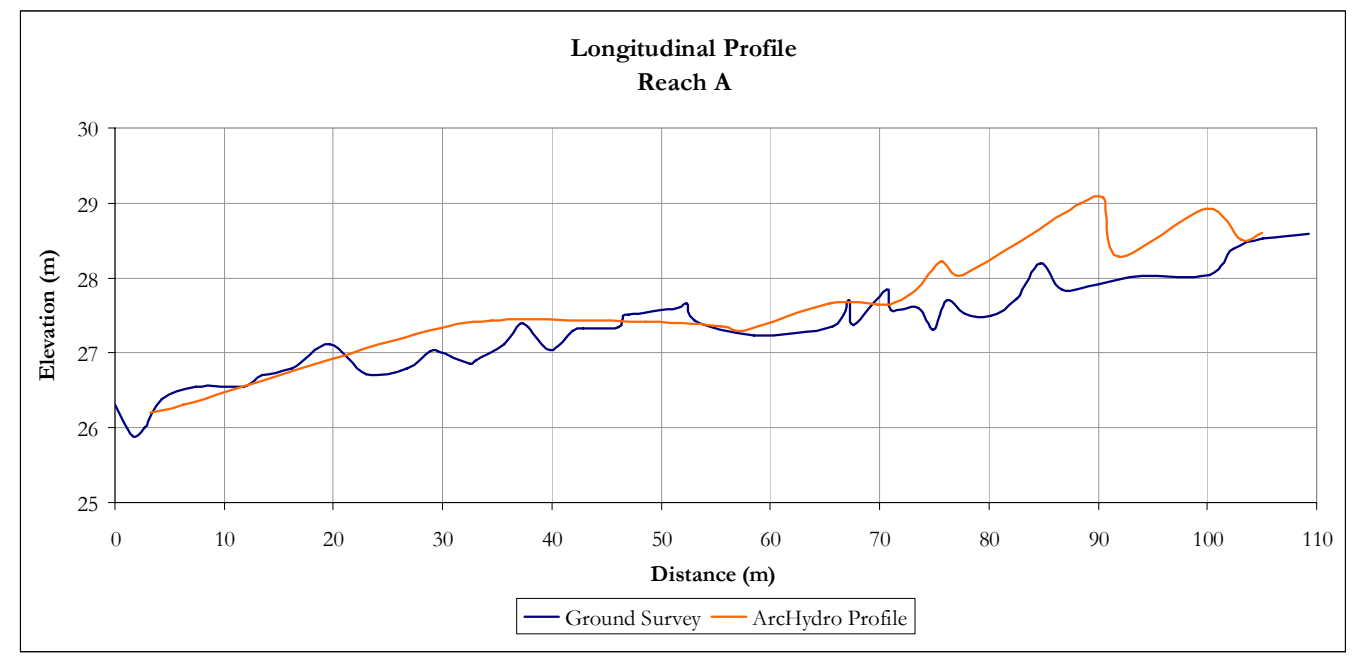

Figure 40. Little Creek Reach A.

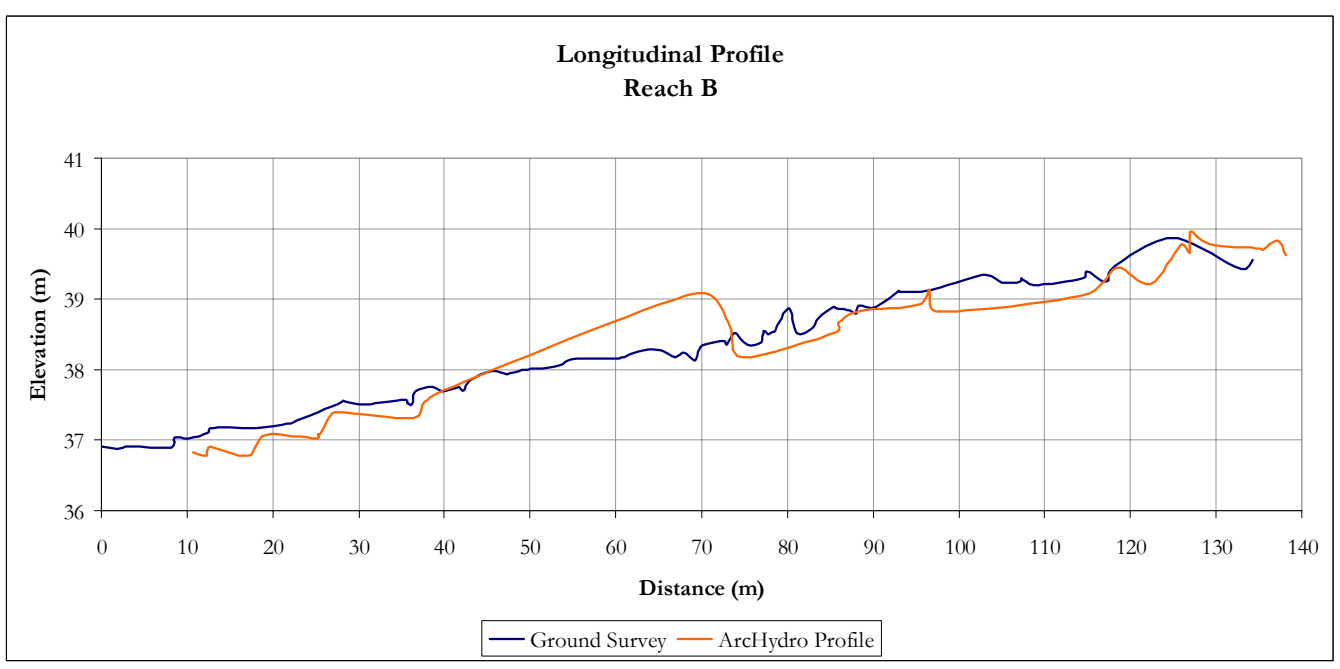

Figure 41. Little Creek Reach B.

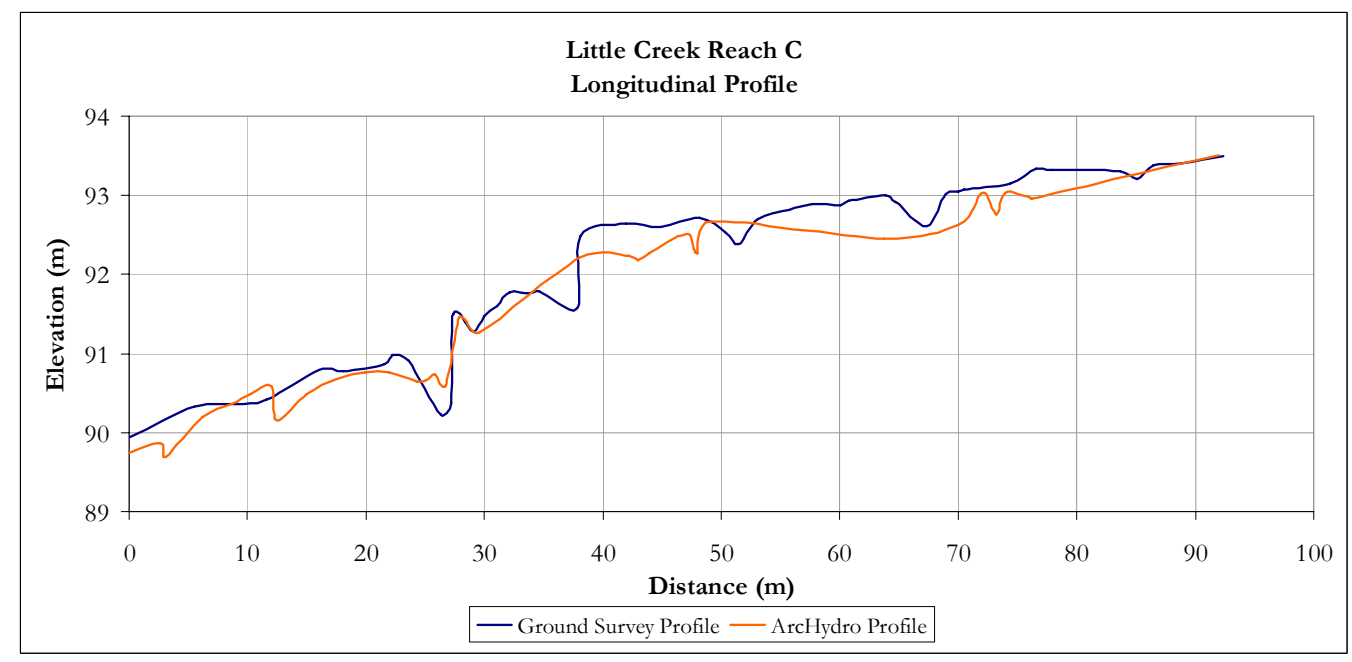

Figure 42. Little Creek Reach C. 


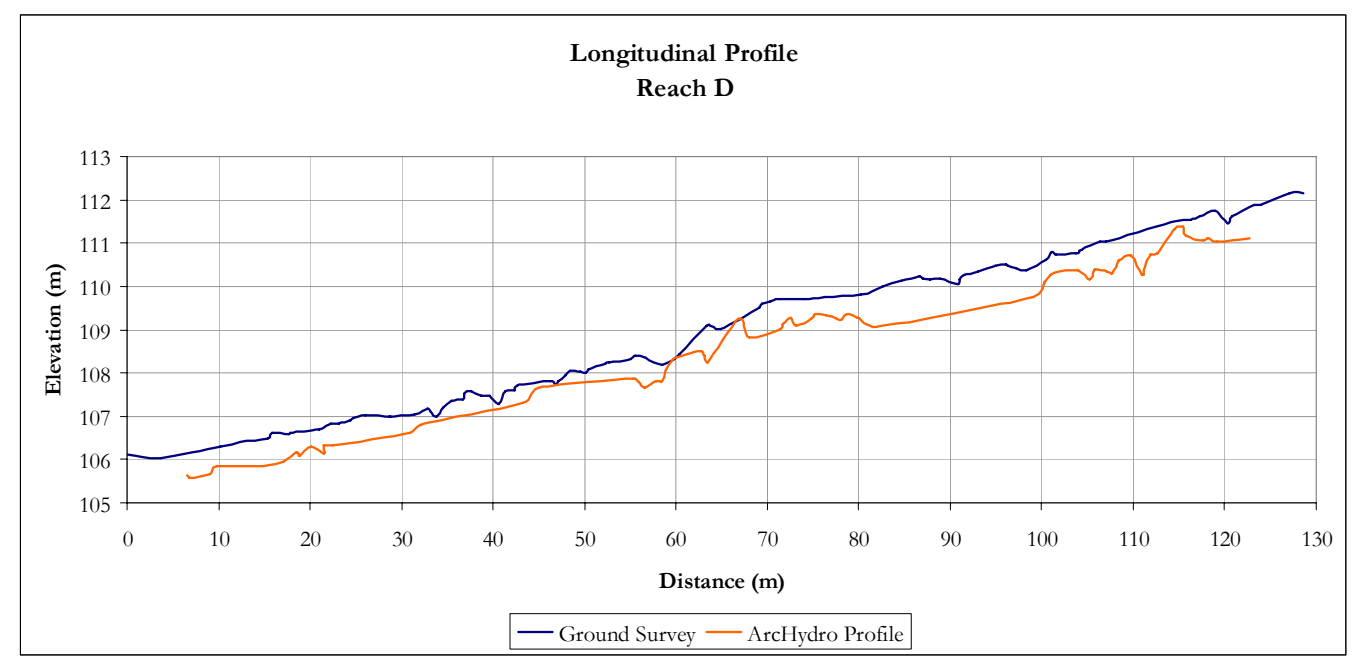

Figure 43. Little Creek Reach D.

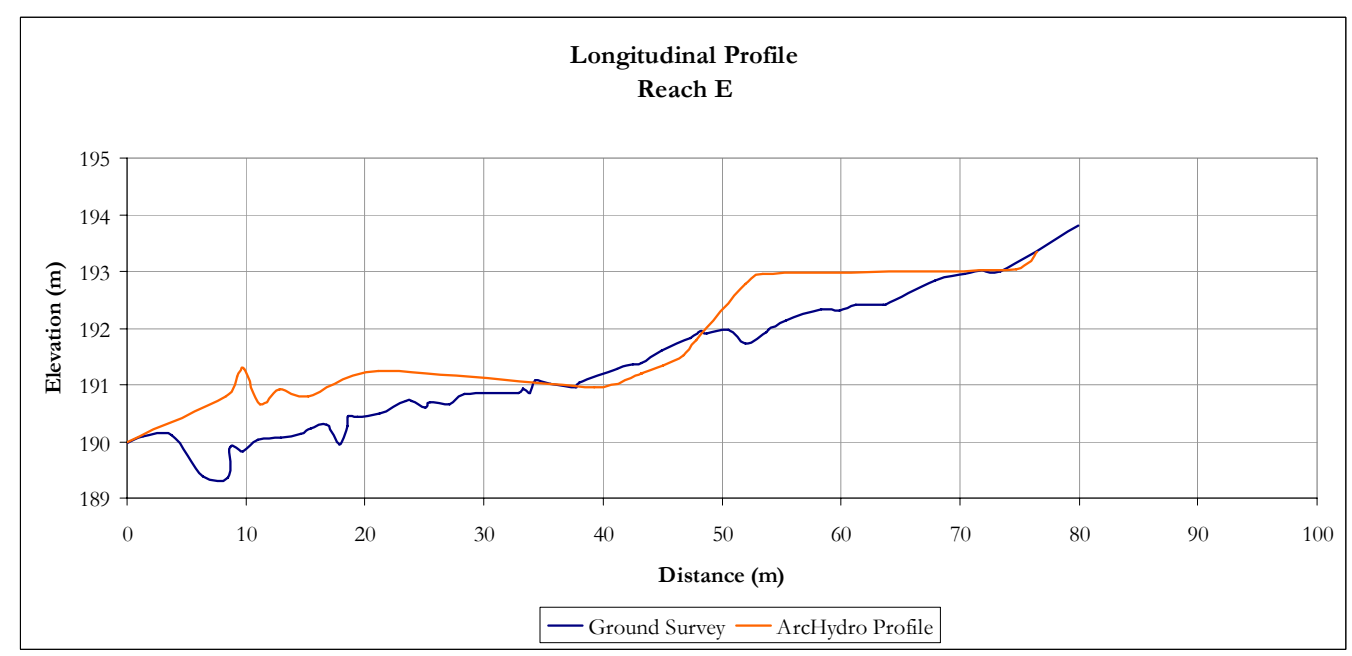

Figure 44. Little Creek Reach E.

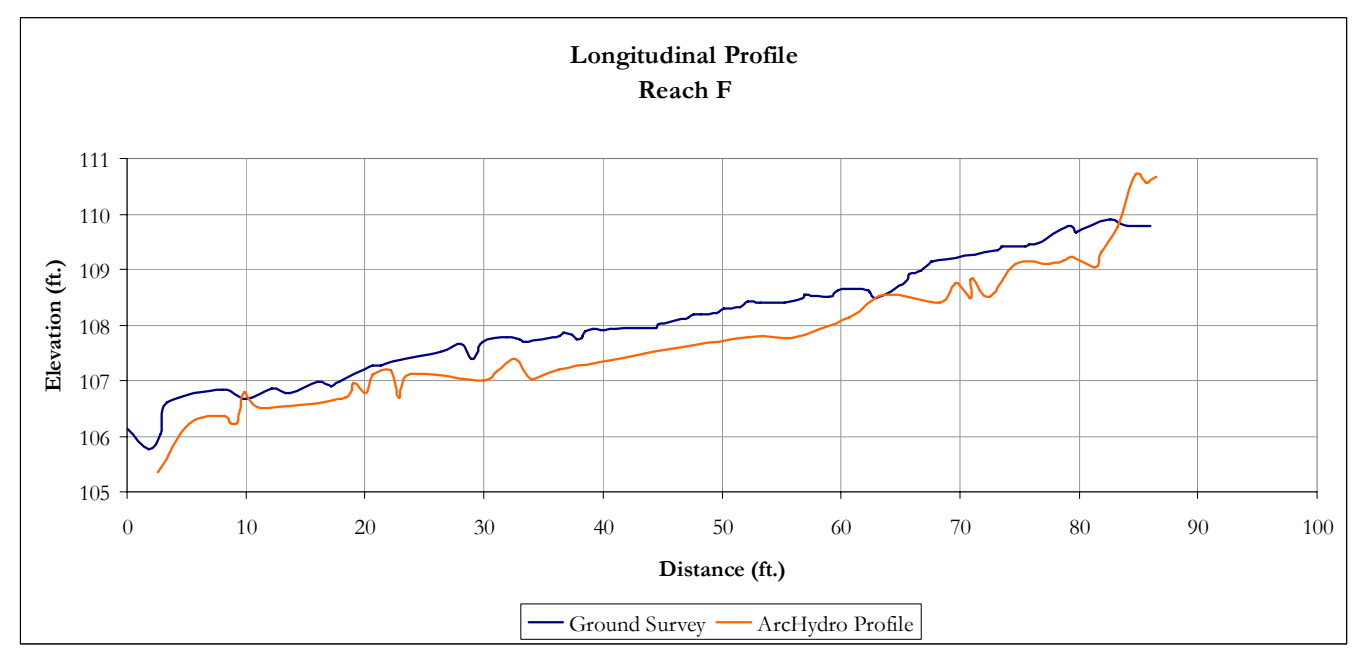

Figure 45. Little Creek Reach F. 
Appendix C.

LiDAR-Derived Road Cross-Sections:

Examples from the Little Creek Road 


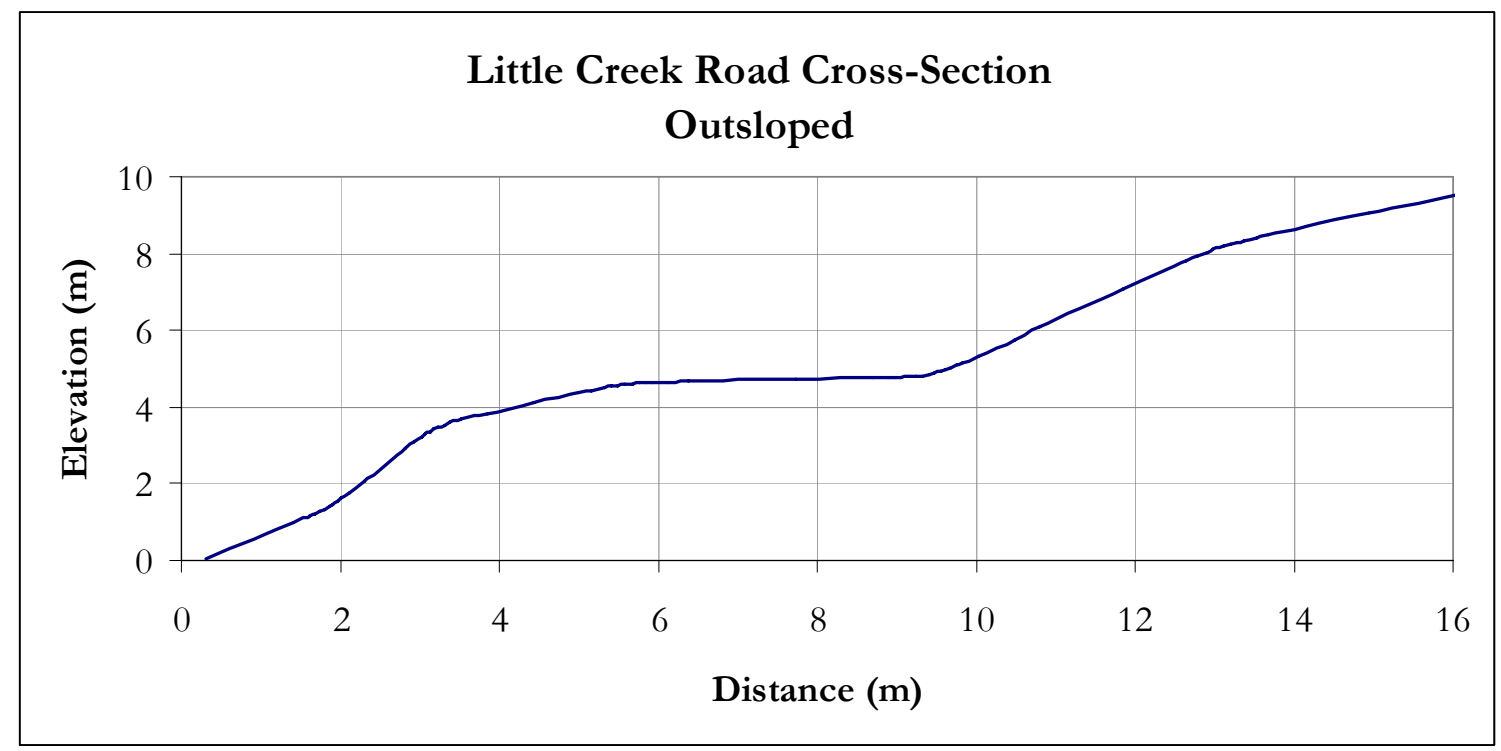

Figure 46. LiDAR-derived road cross-section at outsloped road segment.

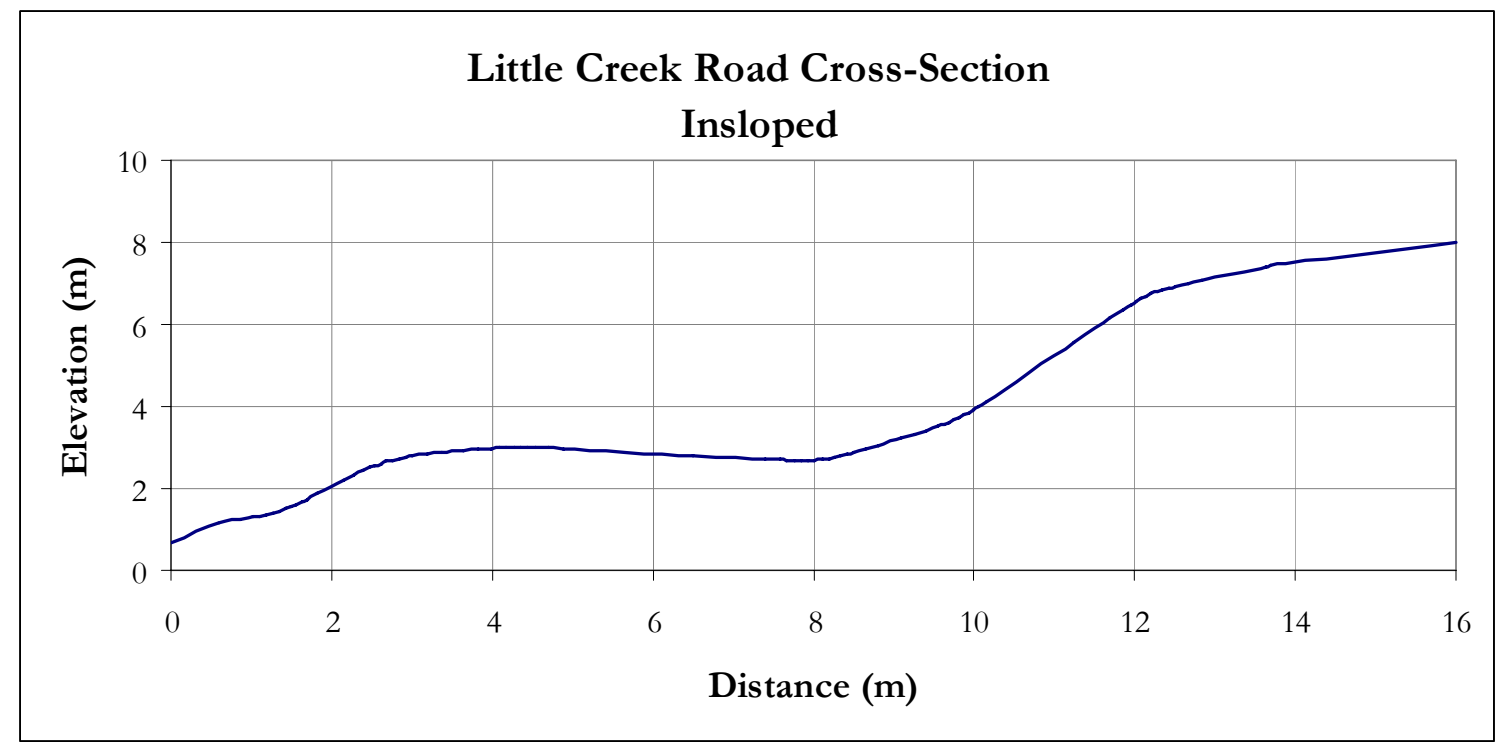

Figure 47. LiDAR-derived road cross-section at an insloped road segment. 


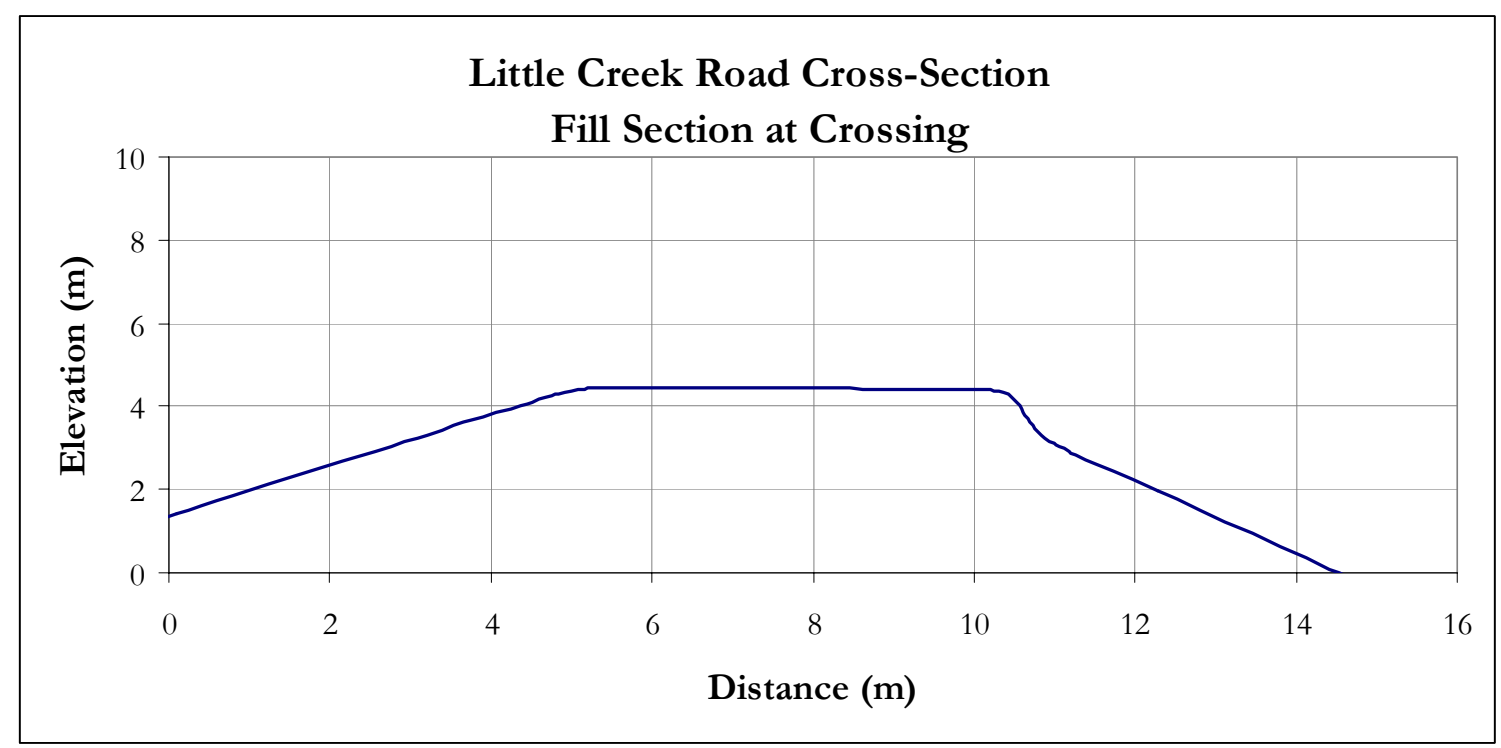

Figure 48. LiDAR-derived road cross-section at a fill crossing.

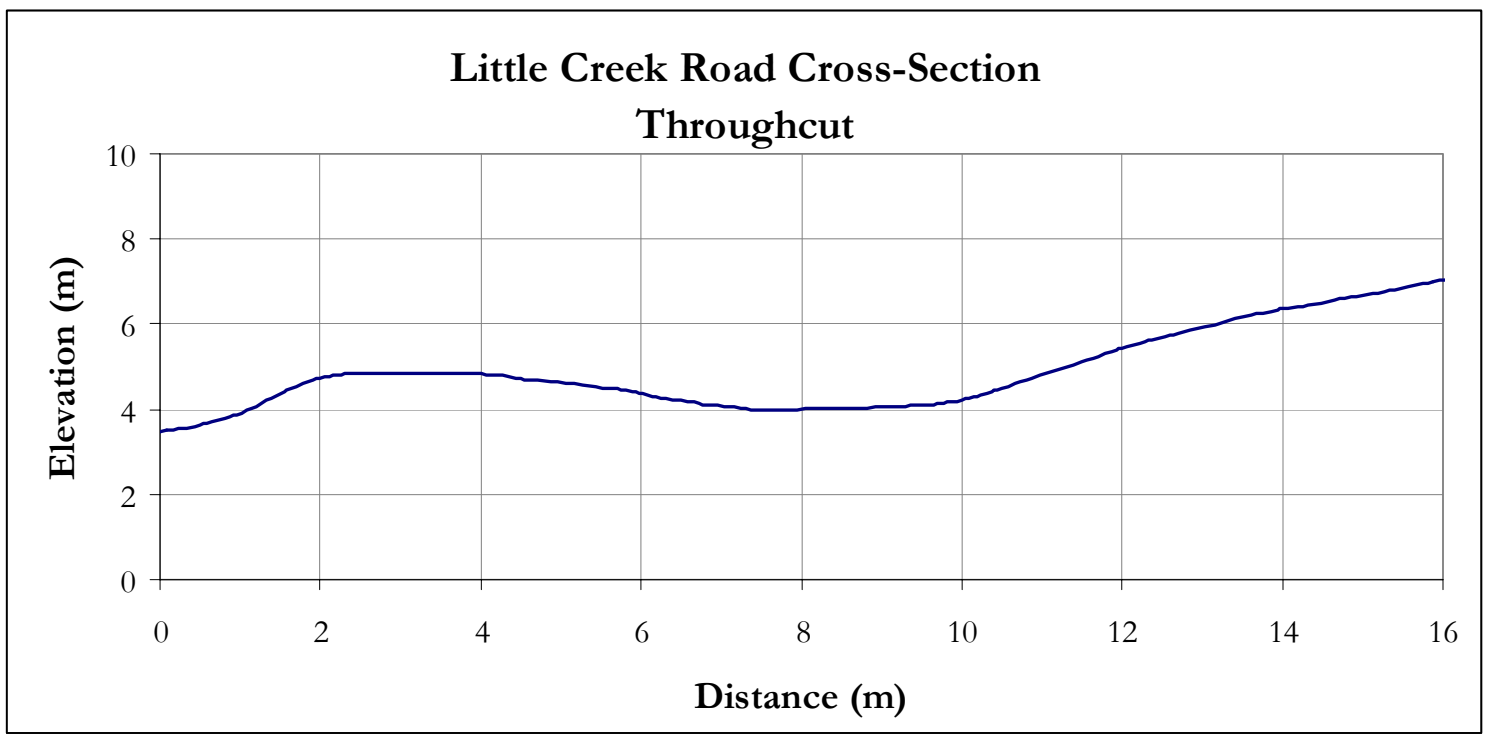

Figure 49. LiDAR-derived road cross-section at a throughcut road segment. 